\title{
Probing the $\mu \nu$ SSM with light scalars, pseudoscalars and neutralinos from the decay of a SM-like Higgs boson at the LHC
}

\author{
Pradipta Ghosh, ${ }^{a, b}$ Daniel E. López-Fogliani, ${ }^{c}$ Vasiliki A. Mitsou, ${ }^{d}$ Carlos Muñoz ${ }^{a, b}$ \\ and Roberto Ruiz de Austri ${ }^{d}$ \\ ${ }^{a}$ Departamento de Fúsica Teórica, Universidad Autónoma de Madrid, \\ Cantoblanco, 28049 Madrid, Spain \\ ${ }^{b}$ Instituto de Física Teórica UAM-CSIC, Campus de Cantoblanco, \\ 28049 Madrid, Spain \\ ${ }^{c}$ Departamento de Física, Universidad de Buenos Aires \& IFIBA-CONICET, \\ 1428 Buenos Aires, Argentina \\ ${ }^{d}$ Instituto de Física Corpuscular CSIC-UV, c/ Catedrático José Beltrán 2, \\ 46980 Paterna, Valencia, Spain \\ E-mail: pradipta.ghosh@uam.es, daniel.lopez@df.uba.ar, \\ vasiliki.mitsou@ific.uv.es, carlos.munnoz@uam.es, rruiz@ific.uv.es
}

ABSTRACT: The " $\mu$ from $\nu$ " supersymmetric standard model ( $\mu \nu \mathrm{SSM})$ can accommodate the newly discovered Higgs-like scalar boson with a mass around $125 \mathrm{GeV}$. This model provides a solution to the $\mu$-problem and simultaneously reproduces correct neutrino physics by the simple use of right-handed neutrino superfields. These new superfields together with the introduced $R$-parity violation can produce novel and characteristic signatures of the $\mu \nu \mathrm{SSM}$ at the LHC. We explore the signatures produced through two-body Higgs decays into the new states, provided that these states lie below in the mass spectrum. For example, a pair produced light neutralinos depending on the associated decay length can give rise to displaced multi-leptons/taus/jets/photons with small/moderate missing transverse energy. In the same spirit, a Higgs-like scalar decaying to a pair of scalars/pseudoscalars can produce final states with prompt multi-leptons/taus/jets/photons.

KEYWORDS: Supersymmetry Phenomenology

ARXIV EPRINT: 1410.2070 


\section{Contents}

1 Introduction 1

2 The model 4

3 Phenomenology of the light neutral states 5

$\begin{array}{lll}3.1 & \text { Light scalars/pseudoscalars } & 7\end{array}$

$\begin{array}{lll}3.2 & \text { Light neutralinos } & 10\end{array}$

4 The SM-like Higgs in the $\mu \nu$ SSM $\quad 13$

5 Masses of the singlet-like states in the $\mu \nu \mathrm{SSM} \quad 18$

5.1 Regions of the parameter space with light scalars, pseudoscalars and neu$\begin{array}{ll}\text { tralinos } & 23\end{array}$

6 New decays of the SM-like Higgs in the $\mu \nu$ SSM 30

$\begin{array}{lll}7 & \text { Conclusions } & 41\end{array}$

\section{Introduction}

The ATLAS and CMS collaborations have finally discovered a new scalar boson $[1,2]$ of mass about $125 \mathrm{GeV}$ at the LHC [1-5]. This new scalar has properties [4-17] similar to that of the much awaited standard model (SM) Higgs boson. However, issues like missing precise experimental measurements over all the SM decay modes (e.g., $b \bar{b}$ ), hitherto existing mild excess in the di-photon channel [12, 17-19], etc., keep the possibility of having a beyond SM origin alive to date. Among a plethora of candidate beyond the SM theories, weak scale supersymmetry (SUSY) has extensively been analysed over a long period of time. Missing experimental evidence of SUSY to date [20, 21], especially when the experimental observations are interpreted with the simplified models, together with a class of theoretical issues, motivates one to consider models beyond the minimal structure.

The " $\mu$ from $\nu$ " supersymmetric standard model $(\mu \nu \mathrm{SSM})[22,23]$ solves the $\mu$ problem [24] of the minimal supersymmetric standard model (MSSM) (see [25-28] for reviews) and simultaneously accommodates the correct neutrino physics [22, 23, 29-34], as guided by the three flavour global neutrino data [35-37]. A set of three right-handed neutrino superfields has been utilised to address both purposes, relating the origin of the $\mu$-term to the origin of neutrino masses and mixing. As a consequence of the construction, the $R$-parity [38-47] is explicitly broken $\left(\mathbb{R}_{p}\right)$ in the $\mu \nu \mathrm{SSM}$. Non-zero neutrino masses in this model appear through a dynamically generated electroweak-scale seesaw [22, 23, 2934]. Thus, the only scale associated with the $\mu \nu \mathrm{SSM}$ is the scale of electroweak symmetry 
breaking (EWSB) or in other words the scale of the soft SUSY-breaking terms, which is in the ballpark of a $\mathrm{TeV}$. This nice feature can produce realistic signatures of this model at colliders [29, 30, 48-51], well verifiable at the LHC or at upcoming accelerator experiments [52-56]. As a consequence of $\not_{p}$, the lightest supersymmetric particle (LSP) is no longer a valid candidate for cold dark matter. Nevertheless, embedding the model in the context of supergravity (see ref. [25] for a review) one can accommodate the gravitino [57] as an eligible decaying dark matter candidate with a life-time greater than the age of the Universe. Its detection is also possible in principle through the observation of a gamma-ray line in the Fermi satellite [57-60]. In ref. [61], the generation of the baryon asymmetry of the universe was analysed in the $\mu \nu \mathrm{SSM}$, with the interesting result that electroweak baryogenesis can be realised.

In the $\mu \nu \mathrm{SSM}$, the bilinear $\mu \hat{H}_{d} \hat{H}_{u}$ term of the MSSM superpotential is replaced by the trilinear terms $\lambda_{i} \hat{\nu}_{i}^{c} \hat{H}_{d} \hat{H}_{u}$. Here $\hat{\nu}_{i}^{c}$ are the right-handed neutrino superfields, singlets under the SM gauge group. New trilinear terms like $Y_{\nu_{i j}} \hat{H}_{u} \hat{L}_{i} \hat{\nu}_{j}^{c}$, where $Y_{\nu_{i j}}$ are the neutrino Yukawa couplings, are also introduced. An effective $\mu$ term with $\mu_{\mathrm{eff}} \equiv \lambda_{i} \nu_{i}^{c}$ is generated after the successful EWSB, where $\nu_{i}^{c}$ denotes the vacuum expectation value (VEV) acquired by the scalar component of the $i$-th right-handed neutrino superfield. In the same spirit, after the EWSB, $Y_{\nu_{i j}} \hat{H}_{u} \hat{L}_{i} \hat{\nu}_{j}^{c}$ terms generate effective bilinear $\not R_{p}$ parameters as $Y_{\nu_{i j}} \nu_{j}^{c}$. Following the trend, effective Majorana masses for right-handed neutrinos, $2 \kappa_{i j k} \nu_{k}^{c}$, are produced from $\kappa_{i j k} \hat{\nu}_{i}^{c} \hat{\nu}_{j}^{c} \hat{\nu}_{k}^{c}$ terms. The explicit breaking of $R_{p}$ is apparent in all the three above mentioned trilinear terms. The order of magnitude for $\nu_{i}^{c}$ is determined from the soft SUSY-breaking terms. Thus, as emphasised before, along with the aforementioned features, the EWSB scale, the origin of the $\mu$-term and the scale of the right-handed neutrino Majorana masses (instrumental in the generation of neutrino mass through a seesaw mechanism) in the $\mu \nu \mathrm{SSM}$ are connected to the one and only scale of the model, namely the scale of the soft SUSY-breaking terms.

It is worthy to discuss here the number of right-handed neutrino superfields in the $\mu \nu \mathrm{SSM}$. Although it is possible to accommodate the correct neutrino data $[35,36]$ at the tree level $[22,23,29-31]$, provided one works with at least two $\hat{\nu}_{i}^{c}$, we stick to three $\hat{\nu}^{c}$ scenario which appears natural from the SM family symmetry. Nevertheless, the $\mu \nu$ SSM with arbitrary number of right-handed neutrino superfields has also been discussed in the literature [30].

It is well evident that the presence of a set of new couplings in the $\mu \nu \mathrm{SSM}$ will trigger a few new decay modes for a SM Higgs-like scalar provided that the new states are lighter than it. Some of these modes, for example Higgs decay into a new scalar/pseudoscalar pair, are well known for extended models (with or without SUSY) with a singlet [62-94]. The singlet nature ${ }^{1}$ of these states is useful to evade a class of LEP constrains [95-101] as well as constraints from hadron colliders [102-108]. Light states are also constrained from a group of low-energy observables [109-120] where the presence of these states can yield enhanced contribution to some processes, often in an experimentally unacceptable way. These issues will be addressed later with further detail.

\footnotetext{
${ }^{1}$ Throughout this article, a singlet-like state implies a state with singlet composition larger than about $90 \%$.
} 
In the case of SUSY models, an additional decay mode for a Higgs-like scalar into a pair of light neutralinos [121-125] is also a viable option. ${ }^{2}$ In the case of a pair of the lightest neutralinos, this mode contributes to the invisible Higgs decay since the lightest neutralino is usually the LSP for a large region of the parameter space. The latter being neutral $[126,127]$ and stable, leaves only missing transverse momentum $\left(P_{\mathrm{T}}\right)$ signature at colliders. In the $\mu \nu \mathrm{SSM}$, however, with $\not R_{p}$ this mode can lead to displaced leptons/taus/jets(hadronic)/photons at colliders depending on the associated decay length [30, 48-50]. In addition to the displaced objects, signals of the $\mu \nu \mathrm{SSM}$ are accompanied by a small or moderate missing transverse energy $\left(\not_{\mathrm{T}}\right)$, the origin of which relies on the light neutrinos and/or possible mis-measurements. This is an apparent contradiction to $R_{p}$ conserving SUSY scenarios where the stable, neutral and hence undetected LSPs leave their collider imprint in the form of large $\not_{\mathrm{T}}$. Nevertheless, a pure $\not_{\mathrm{T}} / \not_{\mathrm{T}}$ signature is also possible for $\mathbb{R}_{p}$ scenario when a neutralino LSP, being lighter than $40 \mathrm{GeV}$, decays beyond the detector coverage [30] or decays to three neutrino final states.

The rich collider phenomenology of the $\mu \nu \mathrm{SSM}$ with $R_{p}$ and extra superfields makes it absolutely legitimate to ask two of the most appealing possibilities, namely:

1. How much room do we have for non-standard (non SM-like) decays of the newly discovered Higgs-like scalar boson with a mass about $125 \mathrm{GeV}$ ?

It is well known that so far ATLAS and CMS collaborations have not observed any significant deviation from the SM expectations while analysing this $125 \mathrm{GeV}$ scalar $[4,7,8]$. The window of non-standardness, however, is not closed to date, e.g. the mild excess in the di-photon decay mode remains in the ATLAS measurements [12, 19] and now is also supported by the CMS results $[17,18]$. At the same time a precise estimation of the total decay width of this scalar is still missing [15, 128130]. Furthermore, missing precision information about all the SM decay modes (e.g., $b \bar{b}[4,131,132]$ and also $\tau^{+} \tau^{-}[4,14,132,133]$ to some extent) allows a big open window for the branching fraction of the non-standard decay modes to date [12, 134148]. Thus, it is rather crucial to investigate these new modes systematically even before developing a linear collider.

2. Experimentally allowed singlet-like light scalars, pseudoscalars and neutralinos are well affordable in the $\mu \nu \mathrm{SSM}[23,30,50]$. So, what will be the consequences of these light states at colliders? For example, how these states can affect the decay phenomenology of other heavier SM/SUSY particles? See ref. [51] for example.

The enriched spectrum of the $\mu \nu \mathrm{SSM}$, as introduced in refs. [22, 23], admits the aforesaid novel Higgs decays which have already been addressed in refs. [30, 48-50]. Further, detail collider analyses for a Higgs-like scalar decaying into a pair of neutralinos have also been discussed in refs. $[48,50]$. However, a concise yet complete description of the resultant phenomenology involving those light states is missing to date and this is exactly what we

\footnotetext{
${ }^{2}$ If allowed kinematically a Higgs-like scalar can also decay into a pair of heavier neutralinos. For example, into a pair of next-to lightest neutralinos. This scenario is constrained from the measured $Z$ decay width for neutralino mass $\lesssim M_{Z} / 2$.
} 
aim to address in the current article in the light of a Higgs-like scalar discovery. Note that, as stated above in point 2, those light states can also modify final state particle multiplicity/signal topology when appear in the decay cascades of SUSY particles. Such analyses are beyond the theme of the current paper and we hope to address them elsewhere.

The paper is organised as follows. We start with a brief description of the model in section 2. A complete overview of all the possible final states at colliders together with the identification of crucial backgrounds, when the SM-like Higgs boson in the $\mu \nu$ SSM decays into a pair of light scalars/pseudoscalars/neutralinos, is discussed in section 3. In section 4 we present a discussion about the tree-level SM-like Higgs boson mass followed by the effect and relevance of loop corrections in the light of a Higgs-like scalar with a mass around $125 \mathrm{GeV}$. Additionally, we also identify the crucial set of parameters. Following this discussion, in section 5 with approximate analytical formulae we identify the set of most relevant parameters and discuss how they determine the masses of those light states. In section 6 , we investigate the relevance of these parameters in controlling the decays of the SM-like Higgs boson into a pair of light states, covering all possible new two-body decays. We also derive the expressions of the decay widths for the new decay modes and also evaluate the same for the SM modes, in the presence of new physics. Finally, we also estimate the various reduced signals strengths in the presence of new decays and compare them with the experimentally measured values. We elaborate our analysis over relevant regions of the parameter space also in the same section. Our concluding remarks are summarised and presented in section 7 .

\section{The model}

The $\mu \nu \mathrm{SSM}$ superpotential following the line of refs. [22, 23] is given by

$$
\begin{aligned}
W= & \epsilon_{a b}\left(Y_{u_{i j}} \hat{H}_{u}^{b} \hat{Q}_{i}^{a} \hat{u}_{j}^{c}+Y_{d_{i j}} \hat{H}_{d}^{a} \hat{Q}_{i}^{b} \hat{d}_{j}^{c}+Y_{e_{i j}} \hat{H}_{d}^{a} \hat{L}_{i}^{b} \hat{e}_{j}^{c}+Y_{\nu_{i j}} \hat{H}_{u}^{b} \hat{L}_{i}^{a} \hat{\nu}_{j}^{c}\right) \\
& -\epsilon_{a b} \lambda_{i} \hat{\nu}_{i}^{c} \hat{H}_{d}^{a} \hat{H}_{u}^{b}+\frac{1}{3} \kappa_{i j k} \hat{\nu}_{i}^{c} \hat{\nu}_{j}^{c} \hat{\nu}_{k}^{c},
\end{aligned}
$$

where $i, j, k$ are family indices and $\epsilon_{12}=1$. Here $R_{p}$ is the combined effect of the $4^{\text {th }}, 5^{\text {th }}$ and $6^{\text {th }}$ terms. It is worthy to note in this connection that in the limit $Y_{\nu} \rightarrow 0, \hat{\nu}^{c}$ can be identified as a pure singlet superfield without lepton number, similar to the next-to minimal supersymmetric standard model (NMSSM, see ref. [149] for a review), where $R_{p}$ is not broken. Thus $R_{p}$ is small since the electroweak-scale seesaw implies small values for the neutrino Yukawa couplings, $Y_{\nu} \sim 10^{-6}-10^{-7}[22,23,29-32]$. This minimal superpotential of eq. (2.1) serves both the purposes of solving the $\mu$-problem and generating non-zero neutrino masses and mixing, as already mentioned in the introduction. Although conventional trilinear $R_{p}$ terms are absent from the superpotential, the leptonic ones can, however, appear through loop processes as shown in ref. [23].

Working in the framework of supergravity, the Lagrangian $\mathcal{L}_{\text {soft }}$ containing the soft supersymmetry breaking terms is given by $[22,23]$ :

$$
\begin{aligned}
-\mathcal{L}_{\text {soft }}= & \left(m_{\widetilde{Q}}^{2}\right)_{i j} \widetilde{Q}_{i}^{a^{*}} \widetilde{Q}_{j}^{a}+\left(m_{\widetilde{u}^{c}}^{2}\right)_{i j} \widetilde{u}_{i}^{c^{*}} \widetilde{u}_{j}^{c}+\left(m_{\widetilde{d}^{c}}^{2}\right)_{i j}{\widetilde{d_{i}^{c}}}_{i}^{*} \widetilde{d}_{j}^{c}+\left(m_{\widetilde{L}}^{2}\right)_{i j} \widetilde{L}_{i}^{a^{*}} \widetilde{L_{j}^{a}} \\
& +\left(m_{\widetilde{e}^{c}}^{2}\right)_{i j} \widetilde{e}_{i}^{c^{*}} \widetilde{e}_{j}^{c}+m_{H_{d}}^{2} H_{d}^{a^{*}} H_{d}^{a}+m_{H_{u}}^{2} H_{u}^{a^{*}} H_{u}^{a}+\left(m_{\widetilde{\nu}^{c}}^{2}\right)_{i j} \widetilde{\nu}_{i}^{c^{*}} \widetilde{\nu}_{j}^{c}
\end{aligned}
$$




$$
\begin{aligned}
& +\epsilon_{a b}\left[\left(A_{u} Y_{u}\right)_{i j} H_{u}^{b} \widetilde{Q}_{i}^{a} \widetilde{u}_{j}^{c}+\left(A_{d} Y_{d}\right)_{i j} H_{d}^{a} \widetilde{Q}_{i}^{b} \widetilde{d}_{j}^{c}+\left(A_{e} Y_{e}\right)_{i j} H_{d}^{a} \widetilde{L}_{i}^{b} \widetilde{e}_{j}^{c}+\text { H.c. }\right] \\
& +\left[\epsilon_{a b}\left(A_{\nu} Y_{\nu}\right)_{i j} H_{u}^{b} \widetilde{L}_{i}^{a} \widetilde{\nu}_{j}^{c}-\epsilon_{a b}\left(A_{\lambda} \lambda\right)_{i} \widetilde{\nu}_{i}^{c} H_{d}^{a} H_{u}^{b}+\frac{1}{3}\left(A_{\kappa} \kappa\right)_{i j k} \widetilde{\nu}_{i}^{c} \widetilde{\nu}_{j}^{c} \widetilde{\nu}_{k}^{c}+\text { H.c. }\right] \\
& -\frac{1}{2}\left(M_{3} \widetilde{\lambda}_{3} \widetilde{\lambda}_{3}+M_{2} \widetilde{\lambda}_{2} \widetilde{\lambda}_{2}+M_{1} \widetilde{\lambda}_{1} \widetilde{\lambda}_{1}+\text { H.c. }\right),
\end{aligned}
$$

where the last term of the $2^{\text {nd }}$ line and all terms appearing in the $4^{\text {th }}$ line are generic to the $\mu \nu \mathrm{SSM}$. Remaining soft terms are the same as those of the MSSM, but without the $\mu B_{\mu} \hat{H}_{u} \hat{H}_{d}$ term.

With the choice of CP-conservation, ${ }^{3}$ VEVs acquired by neutral scalars are given by

$$
\left\langle H_{d}^{0}\right\rangle=v_{d}, \quad\left\langle H_{u}^{0}\right\rangle=v_{u}, \quad\left\langle\widetilde{\nu}_{i}\right\rangle=\nu_{i}, \quad\left\langle\widetilde{\nu}_{i}^{c}\right\rangle=\nu_{i}^{c} .
$$

As already stated, it is apparent that after the EWSB from $4^{\text {th }}$ and $5^{\text {th }}$ terms of eq. (2.1) one can extract the effective $R_{p}$ terms $\left(\varepsilon_{i}\right)$, like in the bilinear $R_{p}$ violating $(\mathrm{BRpV})$ model (see ref. [150] for a review) and the $\mu$ term. They are given by $\sum Y_{\nu_{i j}} \nu_{j}^{c}$ and $\sum \lambda_{i} \nu_{i}^{c}$, respectively.

A dedicated analysis of the model parameter space with minimisation conditions has been addressed in ref. [23]. Also the relative importance of various parameters in the different regions of the parameter space has been discussed there. The enhanced mass matrices are presented in refs. [23, 29, 32]. Augmentation of the mass matrices in the $\mu \nu \mathrm{SSM}$ over the same for the MSSM is a consequence of the additional superfield content and $R_{p}$.

Being elucidate for the convenience of reading, let us mention that the enhancement of the neutral and the charged Higgs sectors occur through the mixing between the neutral and the charged doublet Higgses with the three generations of left- and right-handed sneutrinos, and left- and right-handed charged sleptons, respectively. In a similar way, the mixing among the neutral higgsinos and gauginos with the three families of left- and right-handed neutrinos enlarges the number of neutralino states. An analogous effect for the chargino sector appears through the mixing of the charged higgsino and wino with the charged leptons.

Before we address a Higgs-like scalar boson in the $\mu \nu \mathrm{SSM}$ in the light of Higgs boson discovery and identify the key parameters to accommodate the light scalar, pseudoscalar and neutralino states, it will be convenient to illustrate first their possible collider phenomenology. In this way, the motivation to analyse these states further becomes apparent and we aim to address this in the next section with a complete overview of all the possible collider signatures.

\section{Phenomenology of the light neutral states}

In this section we address the collider phenomenology of all the neutral states lighter than the newly discovered scalar with SM Higgs-like properties and a mass about $125 \mathrm{GeV}$.

\footnotetext{
${ }^{3} \mu \nu \mathrm{SSM}$ with spontaneous CP-violation has been studied in ref. [31].
} 
Further, we also discuss how the presence of these light states can impinge the decay kinematics of the SM-like Higgs boson and produces unconventional signals at colliders. So we focus on the scenario when the decay of a Higgs-like scalar into a pair of light states is completely on-shell. Furthermore, for simplicity we assume that all the allowed light scalar, pseudoscalar and neutralino states are closely spaced in masses, such that an additional decay cascade [49] among these states remains kinematically forbidden. In order to continue our discussions on the light neutral states, a prior and brief description of the mass spectrum would appear very relevant for the convenience of reading.

Following ref. [23], all the eight CP-even neutral scalars are denoted by $S_{\alpha}^{0}$ while $P_{\alpha}^{0}$ stands for the seven CP-odd neutral scalars. In order to address the decay phenomenology of the SM-like Higgs boson into non-standard modes, one needs states lighter than its mass. Naturally singlet-like (i.e., right-handed sneutrino and neutrino-like) states are the experimentally preferred possibility to meet this requirement. These light scalar CP-even and CP-odd states are labeled by $S_{i}^{0}$ and $P_{i}^{0}$, respectively. In this article the indices $i, j, k$ are used to represent generation indices. With this kind of hierarchy in the mass spectrum, $S_{4}^{0}$ represents [48-50] the newly discovered SM Higgs-like scalar state. The seven colour-singlet charged scalar states and the five chargino states are represented by $S_{\alpha}^{ \pm}$and $\tilde{\chi}_{\alpha}^{ \pm}$, respectively. Concerning neutralinos, we use $\widetilde{\chi}_{\alpha}^{0}$ as the generic symbol for the ten neutralino states. The three lightest neutralinos, namely $\widetilde{\chi}_{1,2,3}^{0}$, are nothing but the three light active neutrinos and henceforth will be denoted as $\widetilde{\chi}_{i}^{0}$. Thus, for the $\mu \nu \mathrm{SSM}$ the fourth neutralino state, namely $\widetilde{\chi}_{4}^{0}$, is the lightest neutralino in true sense. In the same spirit, the three lightest charginos, i.e. $\widetilde{\chi}_{i}^{ \pm}$with $i=1,2,3$, coincide with the charged leptons, $e, \mu$ and $\tau$, respectively, with $\widetilde{\chi}_{4}^{ \pm}$representing the true lightest chargino.

We start our discussion with the light scalars and pseudoscalars and successively continue with the light neutralinos. As already stated, we also address the effect of these states in the decays of the SM-like Higgs boson. These new decays are an important probe for new physics since they generate unusual signals at colliders. In addition, these decays are also the leading production sources for these lighter states, since their direct production is suppressed due to the singlet nature. One should note that the direct production rate for these states can be enhanced with the increasing doublet admixture. However, in this way the states may get heavier and hardly produce any unusual decay channels. At the same time, as stated in the introduction, increasing doublet composition makes it harder for these states to evade a class of collider constraints. Additional constraints for these light states, especially for the light pseudoscalar, can appear from their connection to a class of low-energy observables [109-120]. Some of the constraints can be evaded with low $\tan \beta$ $\left(=v_{u} / v_{d}\right)$ values, e.g. $\tan \beta \lesssim 10$ [113], while a correct balance of the singlet-doublet admixing provides an extra handle for the others. As an example of the latter, the branching ratio $(\mathrm{Br})$ of $B_{s}^{0} \rightarrow \mu^{+} \mu^{-}$is sensitive to $1 /\left(m_{P^{0}}\right)^{4}$, where $P^{0}$ represents a generic pseudoscalar. Thus, the scenario with light $P_{i}^{0}$ apparently enhances $\operatorname{Br}\left(B_{s}^{0} \rightarrow \mu^{+} \mu^{-}\right)$and thereby, seems to be excluded by the experimental results. In reality however, as long as the amount of doublet mixing is small, and thus the couplings between the SM particles and these light states are very suppressed due to the dominant singlet nature, these scenarios can escape experimental constraints [87]. Another effect regarding $B_{s}^{0} \rightarrow \mu^{+} \mu^{-}$must be mentioned 
here, since the branching fraction of this process possesses a high power sensitivity to $\tan \beta$ in the numerator while the denominator is sensitive to the high power of the pseudoscalar mass $[151,152]$. Hence, one can either live with small $\tan \beta$ or a heavy pseudoscalar to control the size of $\operatorname{Br}\left(B_{s}^{0} \rightarrow \mu^{+} \mu^{-}\right)$(see refs. [153-160] and references therein). In our analysis we focus on the small $\tan \beta$ values, the most natural option in the presence of light $P_{i}^{0}$.

\subsection{Light scalars/pseudoscalars}

In this subsection we discuss the consequences of the light scalars and/or pseudoscalars in the collider phenomenology of the $\mu \nu \mathrm{SSM}$. Note that the masses of these states must

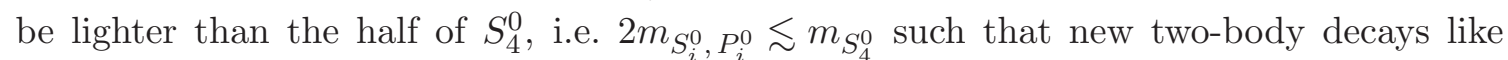
$S_{4}^{0} \rightarrow S_{i}^{0} S_{j}^{0}, P_{i}^{0} P_{j}^{0}$ remain kinematically possible. Subsequent decays of $S_{i}^{0}, P_{i}^{0}$, as will be discussed successively, lead to multi-particle final states. Possible final states strongly depend on the masses of $S_{i}^{0}$ and $P_{i}^{0}$, which are systematically addressed below.

Decaying to leptons and taus: a light scalar/pseudoscalar decaying into a pair of leptons/taus or jets (will be addressed subsequently) occurs essentially due to a small but non vanishing admixture with the doublet Higgs bosons. Final states with electrons are normally suppressed since the couplings of the charged leptons (jets) to the doublet Higgs boson are proportional to their respective masses. The decay into a pair of muons is also normally suppressed for a wide range of $m_{S_{i}^{0}, P_{i}^{0}}$. This specific mode gets sub-leading in the range $2 m_{c} \lesssim m_{S_{i}^{0}, P_{i}^{0}} \lesssim 2 m_{\tau}$, while it dominates in the span of $2 m_{\mu} \lesssim m_{S_{i}^{0}, P_{i}^{0}} \lesssim 2 m_{c}$. The decay into a pair of $\tau$ s gets dominant for $2 m_{\tau} \lesssim m_{S_{i}^{0}, P_{i}^{0}} \lesssim 2 m_{b}$.

$S_{i}^{0}$ and $P_{i}^{0}$ states with masses between $2 m_{\mu}$ to $2 m_{b}$ (i.e., $2 m_{\mu} \lesssim m_{S_{i}^{0}, P_{i}^{0}} \lesssim 2 m_{b}$ ) normally lead to multi-lepton/multi-tau final states at colliders. They are also relatively easy to identify as the number of associated backgrounds are lesser and differentiable. For example, $S_{4}^{0} \rightarrow 2 S_{i}^{0}, 2 P_{i}^{0}$ can easily lead to $4 \mu, 4 \tau$ or $2 \mu 2 \tau$ final states in the $\mu \nu \mathrm{SSM}$ [49]. The $4 \mu$ channel apparently seems to be the most promising one as detection efficiency for muons is rather high at the LHC. This scenario is, however, severely constrained after the recent CMS analyses [107, 108]. The process $P_{i}^{0} \rightarrow \mu^{+} \mu^{-}$itself is also experimentally constrained from the ATLAS results [104]. Further, it is evident from ref. [76] that the typical maximum branching fraction ${ }^{4}$ for a light pseudoscalar decaying to $\mu^{+} \mu^{-}$is $\sim 20 \%$ for $2 m_{\mu} \lesssim m_{S_{i}^{0}, P_{i}^{0}} \lesssim 2 m_{\tau}$. Thus, in general the 4 -muon final state has only $4 \%$ of branching ratio available. This, despite of the large window allowed to date for the $\mathrm{Br}$ of the non-standard/invisible Higgs decays [12, 134-148], would yield poor statistics for $S_{4}^{0} \rightarrow 4 \mu$ process. This drawback, however, can be ameliorated with larger luminosity or moving

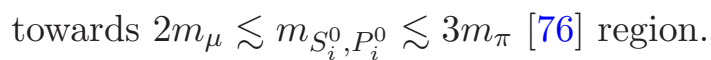

On the contrary, the situation is still experimentally relaxing for $\tau \mathrm{s}$. Although the process pseudoscalar $\rightarrow \tau^{+} \tau^{-}$in the MSSM is constrained from experimental searches $[106,161,162]$, in models with singlet(s) (e.g., the NMSSM or the $\mu \nu \mathrm{SSM}$ ) bypassing the experimental bounds remain possible for the two inter-related reasons:

\footnotetext{
${ }^{4}$ This branching fraction is $\approx 100 \%$ for $2 m_{\mu} \lesssim m_{S_{i}^{0}, P_{i}^{0}} \lesssim 3 m_{\pi}[76]$.
} 
(1) Additional $S_{i}^{0}, P_{i}^{0}$ states must be lighter than $2 m_{b}$ to yield an enhancement for the multi-lepton/multi-tau final states at colliders. Thus, all the four daughter leptons (through $S_{4}^{0} \rightarrow 2 S_{i}^{0}, 2 P_{i}^{0} \rightarrow 2 l^{+} 2 l^{-}, l=\ell(\equiv e, \mu), \tau$ ) are usually not highly boosted and often not well separated from each others. In this situation one might need to adopt modified search criteria to identify these leptons/taus, which are somehow inadequate to date. With existing analysis methods a pair of leptons/taus from such a light $S_{i}^{0}, P_{i}^{0}$ perhaps effectively appears as one single particle [85].

(2) A similar approach with $\tau \mathrm{s}$ is a bit more complicated since for taus the detection efficiency strongly depends on their transverse momentum, $p_{\mathrm{T}}$ [163-165] (see references in [165] also). Normally for low $p_{\mathrm{T}}(\lesssim 30 \mathrm{GeV})$, the $\tau$ detection efficiency falls very sharply [165]. Thus, not all the four taus originating through $S_{4}^{0} \rightarrow 2 S_{i}^{0}, 2 P_{i}^{0} \rightarrow 2 \tau^{+} 2 \tau^{-}$are detectable at the experiment. In addition, proper identification of a $\tau$ as $\tau$-jet occurs only when a $\tau$ decays hadronically, which happens only $18 \%$ of the times with $4 \tau$. Situation with leptonic $\tau$ decays to muon may appear favourable since muon detection efficiency is very high at the LHC. However, this is not a realistic analysis mode since the $\operatorname{Br}(4 \tau \rightarrow 4 \mu)$ is only $\sim 0.1 \%$. In the $\mu \nu \mathrm{SSM}$, however, it is possible to generate mass splittings among the light $S_{i}^{0}, P_{i}^{0}$ states by tuning the relevant parameters [49] so that one of the lighter states decays to di-muon while other(s) to ${ }^{5} \tau^{+} \tau^{-}$. Concerning $\mathrm{Br}$, the $2 \ell 2 \tau$ state is intermediate to $4 \tau \rightarrow 4 e, 4 \mu, 2 e 2 \mu$ and $4 \tau$-jets with $\operatorname{Br}(2 \ell 2 \tau \text {-jets })_{\max } \sim 10 \%$ for $0.5 \mathrm{GeV} \lesssim m_{S_{i}^{0}, P_{i}^{0}} \lesssim 2 m_{\tau}[76]$ although the problem of narrower isolation criterion, as stated already in (1) persists.

It has to be emphasised here that regarding the branching fraction, the state with $4 \tau$-jets dominates over $2 \ell 2 \tau$-jets. The latter, however, is advantageous when $\tau$ detection efficiency is taken into account. Moving towards a different aspect of the final states, the processes $S_{i}^{0}, P_{i}^{0} \rightarrow 2 e, 2 \mu$ theoretically appear with zero $\not_{\mathrm{T}}$, although in reality a nonvanishing $\not_{\mathrm{T}}$ may arise from the possible mis-measurements. On the contrary, $S_{i}^{0}, P_{i}^{0} \rightarrow$ $2 \tau$ state is always accompanied with a non-zero $\not_{\mathrm{T}}$ originating from multiple neutrinos (minimum being 4 for $4 \tau \rightarrow 4 \tau$-jets) which appear in the $\tau$ decay. The presence of four neutrinos, however, does not guarantee a large $\not_{\mathrm{T}}$ due to a possible collinearity among them $[85,87]$.

Decaying to jets: in the same spirit, as stated earlier, the light $S_{i}^{0}, P_{i}^{0}$ states can also decay predominantly into a pair of jets depending on $m_{S_{i}^{0}, P_{i}^{0}}$. These decays are further classified into two groups, (a) a pair of light jets $\left(m_{S_{i}^{0}, P_{i}^{0}} \lesssim 2 m_{\mu}\right)$ including $c \bar{c}, g g$ $\left(2 m_{c} \lesssim m_{S_{i}^{0}, P_{i}^{0}} \lesssim 2 m_{\tau}\right.$ and $m_{S_{i}^{0}, P_{i}^{0}} \rightarrow 0$, respectively) and (b) into $b \bar{b}\left(m_{S_{i}^{0}, P_{i}^{0}} \gtrsim 2 m_{b}\right)$.

The first option (a) has several shortcomings. To start with, this scenario is not generic in $m_{S_{i}^{0}, P_{i}^{0}}$ as in the case of leptonic $(e$ and $\mu$ ) modes. Secondly, the jets produced in this way are narrowly separated just like the earlier discussion with leptons and taus. The third and the most severe issue is to disentangle these jets from the backgrounds. These jets are

\footnotetext{
${ }^{5} \mathrm{~A}$ similar situation is also possible in the NMSSM by a distribution of the different decay Brs. For the $\mu \nu \mathrm{SSM}$ with the three $\hat{\nu}^{c} \mathrm{~s}$, this emerges naturally.
} 
naturally soft as they are originating from the decay of the $S_{4}^{0}$, with a mass about $125 \mathrm{GeV}$. Thus, their information is practically lost within the huge QCD backgrounds, associated with a hadronic collider like the LHC.

Moving towards possibility (b), the processes $S_{i}^{0}, P_{i}^{0} \rightarrow b \bar{b}$ are the most generic decay mode for $S_{i}^{0}, P_{i}^{0}$ over a wide range of $m_{S_{i}^{0}, P_{i}^{0}}$, i.e. $2 m_{b} \lesssim m_{S_{i}^{0}, P_{i}^{0}} \lesssim m_{S_{4}^{0}} / 2$. In addition, with $m_{S_{i}^{0}, P_{i}^{0}} \gg 2 m_{b}$, the produced $b$-jets can be well separated in nature. Further, concerning the backgrounds, one can use the favour of $b$-tagging to discriminate this signature from the backgrounds. The main problem with the $b$-jets is the same as that with the $\tau$-jets, i.e. their detection efficiency is also $p_{\mathrm{T}}$ dependent $[163,166]$. Thus, the process $S_{4}^{0} \rightarrow 4 b-$ jets suffers additional suppression which might lead to a poor statistics. Actually, with a mother particle of about $125 \mathrm{GeV}$ mass, $p_{\mathrm{T}}$ for $3^{\text {rd }}$ and $/$ or $4^{\text {th }} b$-jet can be low enough to fulfil the trigger requirement. One should note that increasing luminosity does not assure a better statistics for this signal, since this also results in a potential growth of the QCD backgrounds. It needs to be emphasised here that one can get higher boost for these jets (leptons/taus) coming from the $S_{i}^{0}, P_{i}^{0}$ states when cascades with heavier particles are considered. However, these processes normally suffer extra suppression from Brs in longer cascades and/or in production cross-section due to the large masses of the concerned particles. Non-zero $\not_{\mathrm{T}}$ can exist for the multi-jet final states, e.g. through semileptonic b-decays.

Decaying to photons: processes like $S_{i}^{0}, P_{i}^{0} \rightarrow \gamma \gamma$ are usually suppressed in Brs due to the singlet nature of the mother particles on top of the loop suppression. Only in the limit of sufficiently light $S_{i}^{0}, P_{i}^{0}\left(\lesssim 3 m_{\pi}\right)$, this mode can lead the race [167]. However, with very small $m_{S_{i}^{0}, P_{i}^{0}}$, just like two earlier scenarios $S_{4}^{0} \rightarrow 2 S_{i}^{0} / 2 P_{i}^{0} \rightarrow 4 \gamma$ [105] will appear as $2 \gamma \mathrm{s}$ at the collider [167]. On the contrary, with heavier $m_{S_{i}^{0}, P_{i}^{0}}$ theoretically a clean $S_{4}^{0} \rightarrow 4 \gamma$ signal is expected. Unfortunately, this situation suffers huge Br suppression $\left(\sim 10^{-5}\right)$ [168]. Hence, unless LHC attains a very high luminosity, this unique channel is hardly recognisable in spite of a negligible associated backgrounds. Theoretical $\not_{\mathrm{T}}$ prediction is zero for this signal. It is to be noted that in a scenario when $S_{i}^{0}, P_{i}^{0}$ are very pure singlets, $\operatorname{Br}\left(S_{i}^{0}, P_{i}^{0} \rightarrow 2 \gamma\right)$ can enhance significantly at the cost of the reduced tree-level couplings to fermions. In this scenario $\operatorname{Br}\left(S_{4}^{0} \rightarrow 4 \gamma\right)$ can rise by orders of magnitude [71].

Decaying to mixed final states: in the NMSSM, depending on the respective Brs and masses, a pair of light scalars/pseudoscalars can decay into two different modes. For example, one of them decays into $\tau^{+} \tau^{-}$while the other into $\mu^{+} \mu^{-} / b \bar{b}$. This way, depending on the mass of the mother particle, one can get mixed final states like $2 \mu 2 \tau, 2 \tau 2 b$, $2 \gamma 2 j$ (light jets) etc. Most of these novel signals are, however, suppressed due to $\mathrm{Br}$ multiplication. Being precise, a scalar/pseudoscalar with mass $\gtrsim 2 m_{b}$ typically has $\operatorname{Br}\left(S^{0} / P^{0} \rightarrow \tau^{+} \tau^{-}\right) \sim 0.1$ and $\operatorname{Br}\left(S^{0} / P^{0} \rightarrow b \bar{b}\right) \gtrsim 0.9$. Thus, the resultant $2 b 2 \tau$ state has an effective suppressed $\mathrm{Br} \sim 9 \%$. On the contrary, for the $\mu \nu \mathrm{SSM}$ a splitting within different $S_{i}^{0}, P_{i}^{0}$ is naturally possible [49]. Hence, with the proper mass scales when one of the $S_{i}^{0}, P_{i}^{0}$ decays to $b \bar{b}$, another one can easily decay to $\tau^{+} \tau^{-}$with $\operatorname{Br} \sim 1$ for both of the modes. This way the $\mu \nu \mathrm{SSM}$ can uniquely escape the problem of $\mathrm{Br}$ suppression as noted in ref. [49]. We note in passing that a similar situation is also affordable in the 
NMSSM with more than one singlet. However, this is a rather forceful construction while in the $\mu \nu \mathrm{SSM}$ the existence of three $\hat{\nu}^{c} \mathrm{~S}$ is well motivated by the SM family symmetry. The amount of $\ddot{H}_{\mathrm{T}}$ associated with these signatures can vary from zero to moderate values, depending on the decay modes.

Finally, to conclude the discussion with the light $S_{i}^{0}, P_{i}^{0}$ states, we describe possible leading backgrounds, without which these analyses would remain incomplete. For all the decay modes mentioned above, the dominant SM backgrounds arise from DrellYan (DY), electroweak di-boson $(W W, W Z, Z Z / \gamma), b \bar{b}$, di-leptonically decaying $t \bar{t}$ and $W / Z+$ jets. Some other sub-leading backgrounds can appear from electroweak tri-boson $(W W W, W W Z, Z Z Z / \gamma), t \bar{t} W / Z$, etc., which may yield sizable contributions with larger centre-of-mass energy $\left(\mathrm{E}_{\mathrm{CM}}\right)$ and higher integrated luminosity $(\mathcal{L})$. These backgrounds can somehow be ameliorated by studying di-jet/di-tau or di-lepton $\mathrm{M}_{\mathrm{T} 2}[169,170] /$ invariant

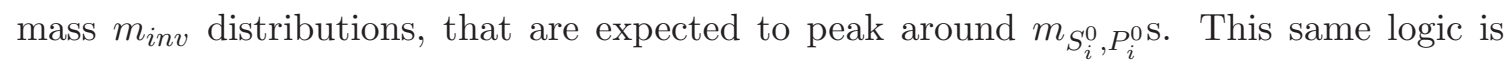
also applicable for the backgrounds arising from the MSSM, but fails for the NMSSM backgrounds. In the NMSSM, just like the $\mu \nu \mathrm{SSM}$, di-lepton or di-jet/di-tau $m_{i n v} / \mathrm{M}_{\mathrm{T} 2}$ distribution can peak around $m_{S_{i}^{0}, P_{i}^{0}}$ and thus, produces irreducible backgrounds to these class of signals. However, if several and non-degenerate singlets are favoured by the nature, then the $\mu \nu \mathrm{SSM}$ can give unique collider signals [49] in terms of the mixed final states. As an example, one can observe two different peaks in the $m_{i n v} / \mathrm{M}_{\mathrm{T} 2}$ distributions corresponding to two different $m_{S_{i}^{0}, P_{i}^{0}}$. A similar scenario is beyond the scope of the standard NMSSM with only one singlet. Note that a NMSSM theory with three $\hat{\nu}^{c} \mathrm{~S}$ [171] produces an irreducible impostor to all the signals of the $\mu \nu \mathrm{SSM}$ even with $R_{p}$ conserving vacua. However, in this case $\not_{\mathrm{T}}$ could be larger and the scenario is constrained from dark matter searches.

\subsection{Light neutralinos}

In this subsection we moved to the study of light neutralinos and their phenomenological consequences in $S_{4}^{0}$ decays. Considering only the on-shell $S_{4}^{0}$ decay, as stated earlier, one concludes $2 m_{\widetilde{\chi}^{0}} \lesssim m_{S_{4}^{0}}$. Clearly, from the lighter chargino mass bound [172] the possible leading composition for such light neutralinos is either bino- or singlino-like (i.e., righthanded neutrino-like) or a bino-singlino mixed state. The chargino mass bound also implies that the minimum of $\left(\mu, M_{2}\right)$ (the parameters that control $\widetilde{\chi}^{ \pm}$mass with $M_{2}$ as the $\mathrm{SU}(2)$ gaugino soft-mass) must be $\gtrsim 100 \mathrm{GeV}$. Further, a bino- [173, 174] or singlino-like [51] nature is also necessary for a light ${ }^{6} \widetilde{\chi}^{0}$ to survive the constraints of measured $Z$-decay width [172]. In this article we stick to a situation where $\widetilde{\chi}_{4,5,6}^{0}$ are singlino-like while $\widetilde{\chi}_{7}^{0}$ is bino-like. In addition, we choose $2 m_{\widetilde{\chi}_{7}^{0}} \gtrsim m_{S_{4}^{0}}$ (will be explained subsequently) and thus, concentrate on singlino-like light neutralinos with ${ }^{7} 2 m_{\widetilde{\chi}_{4,5,6}^{0}} \lesssim m_{S_{4}^{0}}$.

We begin our discussion with the novel aspect of the $\mu \nu \mathrm{SSM}$ to accommodate displaced and yet detectable leptons/taus/jets/photons at colliders [30, 49, 50]. The normal decay modes for the lightest neutralino, $\widetilde{\chi}_{4}^{0}$, is primarily through an electroweak SM gauge boson. However, when $m_{\widetilde{\chi}_{4}^{0}}<M_{W}$, the associated decay lengths are often beyond the charge

\footnotetext{
${ }^{6}$ It has been reported in ref. [175] that a very light neutralino can receive constraints from $B$-physics. However, ref. [176] has argued the absence of any such effects for the MSSM with minimal flavour violation.

${ }^{7}$ For simplicity, we consider singlinos that are quasi-degenerate in masses.
} 


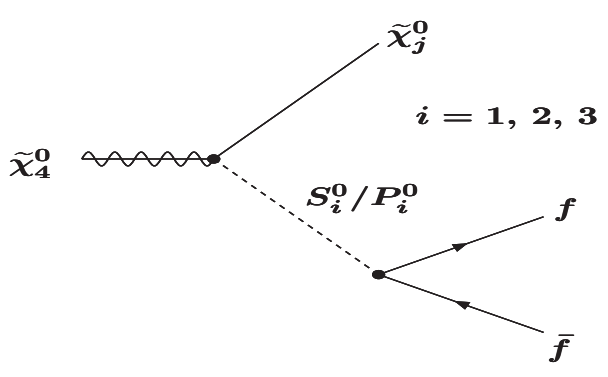

Figure 1. Figure showing $\widetilde{\chi}_{4}^{0} \rightarrow \widetilde{\chi}_{j}^{0}+S_{i}^{0} / P_{i}^{0}$ decay processes, followed by $S_{i}^{0} / P_{i}^{0} \rightarrow f \bar{f}$ decays. with $f$ denoting a possible final state particle, e.g. a lepton/tau/jet/photon etc.

tracker of the LHC, i.e. larger than $1 \mathrm{~m}$, due to the presence of an off-shell intermediate $W^{ \pm}, Z$. Particularly, for $m_{\widetilde{\chi}_{4}^{0}} \lesssim 30 \mathrm{GeV}$ the decays occur outside the detector coverage [30]. Hence, $S_{4}^{0} \rightarrow \widetilde{\chi}_{4}^{0} \widetilde{\chi}_{4}^{0}$ process yields a pure $\not_{\mathrm{T}}$ signal, just like the SUSY models with conserved $R_{p}$. In the $\mu \nu \mathrm{SSM}$ with extended field content one can, however, get lighter $S_{i}^{0}, P_{i}^{0}$ states below $m_{\widetilde{\chi}_{4}^{0}}$ for suitable parameter choices $[30,49]$. Hence, the presence of a new two-body $\widetilde{\chi}_{4}^{0}$ decay like $\widetilde{\chi}_{4}^{0} \rightarrow S_{i}^{0} / P_{i}^{0}+\widetilde{\chi}_{j}^{0}$ can reduce the $\widetilde{\chi}_{4}^{0}$ decay length drastically [30] even when it is very light $[49,50]$. These decay modes are shown in figure 1 . These decays dominate even when $S_{i}^{0} / P_{i}^{0}$ states are slightly heavier than $m_{\widetilde{\chi}_{4}^{0}}$ [50]. An example of this kind, when $S_{4}^{0} \rightarrow \widetilde{\chi}_{4}^{0} \widetilde{\chi}_{4}^{0}$ decay leads to the displaced but detectable multi- $\tau+\not_{\mathrm{T}}$ final state, has already been analysed in ref. [50]. A note of caution has to be emphasised here, i.e. reduction of the decay length in the absence of light $S_{i}^{0}, P_{i}^{0}$ states makes it rather hard for a light $\widetilde{\chi}_{4}^{0}$ in other $\mathbb{R}_{p}$ models to decay within the detector coverage. Nonetheless, for certain values of the concerned couplings, a very light $\widetilde{\chi}_{4}^{0}$ can decay in the range of $1 \mathrm{~cm}-3 \mathrm{~m}$ for MSSM with trilinear $\not R_{p}[150,177]$.

It is now important to address the composition of a light $\widetilde{\chi}_{4}^{0}$. Note that with a simple choice of quasi-degenerate, flavour diagonal $\kappa_{i j k}$, i.e. say $\kappa_{i}$ and universal $\nu^{c}$, one encounters two experimentally viable possibilities, (1) $\widetilde{\chi}_{4,5,6}^{0}$ are singlino-like while $\widetilde{\chi}_{7}^{0}$ is bino-like and (2) a bino-like $\widetilde{\chi}_{4}^{0}$ lies below singlino-like $\widetilde{\chi}_{5,6,7}^{0}$. The U(1) gaugino soft-mass $M_{1}$ is the key parameter to control the mass scale of a bino-like $\widetilde{\chi}^{0}$ and thus a light $\left(\lesssim m_{S_{4}^{0}} / 2\right)$ binolike $\widetilde{\chi}_{4}^{0}$ requires a $M_{1}$ lighter or around $60 \mathrm{GeV}$. Such a small $M_{1}$ value, when considered together with the experimentally hinted scale of gluino mass, i.e. $m_{\widetilde{g}} \gtrsim 1.2 \mathrm{TeV}[20,21]$, requires breaking of the gaugino universality relation. For a singlino, mass scale is determined by $\kappa$ and $\nu^{c}[22,23,29]$. The mass scales for $S_{i}^{0}, P_{i}^{0}$ (see section 5 for details) are mainly governed by $\kappa, \nu^{c}$ and $A_{\kappa}$ parameters [23, 29]. Thus, simultaneous presence of the lighter $S_{i}^{0}, P_{i}^{0}$ states are more feasible with a singlino-like $\widetilde{\chi}_{4}^{0}$ compared to a bino-like $\widetilde{\chi}_{4}^{0}$. Note that singlino-like quasi-degenerate $\widetilde{\chi}_{5,6}^{0}$ can also decay through $S_{i}^{0} / P_{i}^{0}$ as shown in figure 1 . With $\widetilde{\chi}_{4,5,6}^{0}$ closely spaced in masses, one also encounters 3-body decays like $\widetilde{\chi}_{5,6}^{0} \rightarrow \widetilde{\chi}_{4,5}^{0}+\mu^{+} \mu^{-} /$jet pair etc. These final state particles, coming through the off-shell $S_{i}^{0} / P_{i}^{0}$, normally remain experimentally undetected due to their soft-nature [50], although the final state particle multiplicity is rather large. It is possible to evade these soft final states by introducing large splittings among $\kappa_{i}$ s, however, at the cost of an enlarged set of parameters and normally reducing the predictivity of the model. 
Let us now try to justify our choice of $2 m_{\widetilde{\chi}_{7}^{0}} \gtrsim m_{S_{4}^{0}}$. First of all, as already stated, from theoretical prejudice a scenario like $2 m_{\widetilde{\chi}_{7}^{0}} \lesssim m_{S_{4}^{0}}$ for a bino-like $\widetilde{\chi}_{7}^{0}$ requires breaking of the gaugino universality condition at the high scale. Secondly a light $\widetilde{\chi}_{7}^{0}$ naturally enters into $S_{4}^{0}$ decay chains and yield a signal like $S_{4}^{0} \rightarrow 2 \widetilde{\chi}_{7}^{0} \rightarrow 2 \widetilde{\chi}_{4}^{0}+2 S_{i}^{0}, 2 P_{i}^{0} \rightarrow$ a combination of four leptons/taus/jets/photons $+\not_{\mathrm{T}}$. Here $\widetilde{\chi}_{4}^{0}$ decays according to figure 1 . Unfortunately, with a light mother particle like $S_{4}^{0}$, most of these jets/leptons are not well boosted as well as most likely not well isolated. Consequently, most of these novel multi-particle final states remain experimentally undetected. Thus, in this article we mainly discuss about singlet-like $\widetilde{\chi}_{4,5,6}^{0}$ with a bino-like $\widetilde{\chi}_{7}^{0}$ such that $2 m_{\widetilde{\chi}_{7}^{0}} \gtrsim m_{S_{4}^{0}}$. We note in passing that for the sake of completeness we do discuss the scenario with a bino-like $\widetilde{\chi}_{4}^{0}$ while discussing new two-body Higgs decays in section 6 .

Since we stick to $2 m_{\widetilde{\chi}_{4}^{0}} \lesssim m_{S_{4}^{0}}, \widetilde{\chi}_{4}^{0} \rightarrow S_{i}^{0} / P_{i}^{0}+\widetilde{\chi}_{j}^{0}$ remain the leading $\widetilde{\chi}_{4}^{0}$ decay modes, even when $S_{i}^{0}, P_{i}^{0}$ are slightly heavier than $\widetilde{\chi}_{4}^{0}$ [50]. Now it is apparent that the decay products for $\widetilde{\chi}_{4}^{0}$ will trail the same for $S_{i}^{0}, P_{i}^{0}$ as already addressed in the previous subsection. One should note that compared to the prompt decays, the amount of $\not_{\mathrm{T}}$ will be different with two extra neutrinos coming form a pair of $\widetilde{\chi}_{4}^{0}$ decay. A class of possible final states from $S_{4}^{0} \rightarrow 2 \widetilde{\chi}_{4}^{0}$ are $4 b+\not_{\mathrm{T}}, 2 b 2 \tau+\not_{\mathrm{T}}, 2 \mu 2 \tau+\not_{\mathrm{T}}, 2 \gamma 2 j+\not_{\mathrm{T}}$ etc. However, a $\widetilde{\chi}_{4}^{0}$ decay has an extra advantage over the same for $S_{i}^{0}, P_{i}^{0}$, which is the appearance of displaced vertices. In this way the $\mu \nu \mathrm{SSM}$ can produce potentially non-standard signals, e.g. displaced multi-photons at colliders. A displaced multi-photon signal is normally very suppressed for minimal $\not R_{p}$ models since $\widetilde{\chi}_{\mathrm{LSP}}^{0} \rightarrow \widetilde{\chi}_{i}^{0} \gamma$ appears through the one-loop processes $[43,178-180]$. The presence of displaced vertices are useful to reject the possible SM backgrounds efficaciously which are generically prompt. ${ }^{8}$ Prompt SUSY backgrounds are also differentiable in the same fashion. SUSY backgrounds with displaced objects can be separated by constructing the di-lepton/di-jet/di-tau invariant mass $/ \mathrm{M}_{\mathrm{T} 2}$ distribution that peaks around a scalar/pseudoscalar mass with a long tail from possible wrong combinatorics. A possible look-alike can appear from the NMSSM in a fine tuned corner of the parameter space [181, 182]. However, as argued in ref. [182], the appearance of a mesoscopic decay length $(1 \mathrm{~cm}-3 \mathrm{~m})$ is not possible in this scenario. Hence, these signatures remain rather unique to SUSY models with singlets with or without $R_{p}$, e.g. the $\mu \nu \mathrm{SSM}$ or the NMSSM with $3 \hat{\nu}^{c}$ for a range of $m_{\widetilde{\chi}_{4}^{0}}$, although the latter with $R_{p}$ conserving vacua produces larger $\not_{\mathrm{T}}$ and suffers additional constraints from dark matter searches.

To recapitulate, we have addressed the complete relevant phenomenological scenarios that can arise from the light scalars, pseudoscalars and neutralinos. We have also discussed their consequences in $S_{4}^{0}$ decay modes. We are now in the ideal state to identify the set of parameters which assure these light states. However, before that it will be useful to discuss the parameter space in the $\mu \nu \mathrm{SSM}$ that can accommodate a SM-like Higgs with a mass about $125 \mathrm{GeV}$. This is also rather necessary as we aim to explore various light states in the light of the $S_{4}^{0}$ decay that has a mass around $125 \mathrm{GeV}$. One should note that the presence of these light states can also lead to new signals at colliders for other heavier SM

\footnotetext{
${ }^{8}$ Normally this also includes displaced objects from B or D meson decays, unless the boost is very high or the associated $\widetilde{\chi}_{4}^{0}$ decay length is very small.
} 
particles. For example, consequences of the light scalars, pseudoscalars and neutralinos in the $\mu \nu \mathrm{SSM}$ in the decays of $W^{ \pm}$and $Z$ bosons have already been addressed in ref. [51]. We note in passing that, since we aim at covering all phenomenological consequences of the light scalars, pseudoscalars and neutralinos in the SM-like Higgs phenomenology, analyses with numerical examples are beyond the theme of the current work. We will address these issues in a set of forthcoming publications [183].

\section{The SM-like Higgs in the $\mu \nu \mathrm{SSM}$}

After the discovery of a new scalar boson $[1,2]$ with properties like the SM Higgs boson, the constraints on the parameter space and mass spectrum of the SUSY models are severely tightened. It is hence absolutely relevant to re-investigate the $\mu \nu \mathrm{SSM}$ parameter space [23] to accommodate this new scalar and to analyse its general phenomenological consequences respecting various experimental results.

We start with a note on the tree-level analysis of Higgs mass and discuss the effect and relevance of the loop corrections in succession. Further, we also highlight the possible differences of the concerned mass spectrum with that of the MSSM. We want to emphasis here that the analysis presented in this section has notable similarity with that of the NMSSM Higgs sector. However, $\mathbb{R}_{p}$ and an enhanced particle content offer a novel and unconventional phenomenology for the $\mu \nu \mathrm{SSM}[29,30,48-51]$ which deserves a systematic analysis.

At this juncture it is relevant to mention the value of $m_{S_{4}^{0}}$ that will be used to estimate some other relevant quantities in this section. The latest ATLAS result gives $m_{S_{4}^{0}}=$ $125.36 \pm 0.41 \mathrm{GeV}$, after combining the measured values from $S_{4}^{0} \rightarrow Z Z^{*} \rightarrow 4$ leptons and $S_{4}^{0} \rightarrow \gamma \gamma$ decay modes [15]. For the CMS the latest number, after combining the measurements over the same two decay modes, gives $m_{S_{4}^{0}}=125.03_{-0.31}^{+0.29} \mathrm{GeV}[17]$. In this article we choose to work with $m_{S_{4}^{0}}=125 \mathrm{GeV}$ which will be used henceforth. This value of $m_{S_{4}^{0}}$ is within the $1 \sigma$ range of the ATLAS and CMS observations.

In the $\mu \nu \mathrm{SSM}$, as already stated in section 2 , the doublet-like Higgses mix with the three families of the left- and the right-handed sneutrinos. Through the mixing with the right-handed sneutrinos, the lightest doublet-like Higgs mass at the tree-level receives an extra contribution ${ }^{9}$ in the $\mu \nu \mathrm{SSM}$ in such a way that the upper bound is now given by [23]

$$
\left(m_{h}^{\text {tree }}\right)^{2} \leq M_{Z}^{2}\left(\cos ^{2} 2 \beta+\frac{2 \lambda^{2} \cos ^{2} \theta_{W}}{g_{2}^{2}} \sin ^{2} 2 \beta\right) \approx M_{Z}^{2}\left(\cos ^{2} 2 \beta+3.62 \lambda^{2} \sin ^{2} 2 \beta\right),
$$

where $M_{Z}$ denotes the mass of $Z$ boson, $g_{2}$ is the $\mathrm{SU}(2)$ gauge coupling, $\tan \beta=\frac{v_{u}}{v_{d}}$ and $\theta_{W}$ is Weinberg mixing angle. The extra piece of contribution grows with small $\tan \beta$ and large $\boldsymbol{\lambda}\left(\equiv\left|\sqrt{\sum \lambda_{i}^{2}}\right|=\sqrt{3} \lambda\right.$ assuming universal $\lambda_{i}$, which will be used henceforth throughout the text). Equation (4.1) can be written in a more elucidate form as

$$
\left(m_{h}^{\text {tree }}\right)^{2} \leq \frac{M_{Z}^{2}}{\left(1+\tan ^{2} \beta\right)^{2}}\left[\left(1-\tan ^{2} \beta\right)^{2}+14.48 \lambda^{2} \tan ^{2} \beta\right] .
$$

\footnotetext{
${ }^{9}$ A similar feature exists for the NMSSM [184-188], however, with only one $\lambda$.
} 


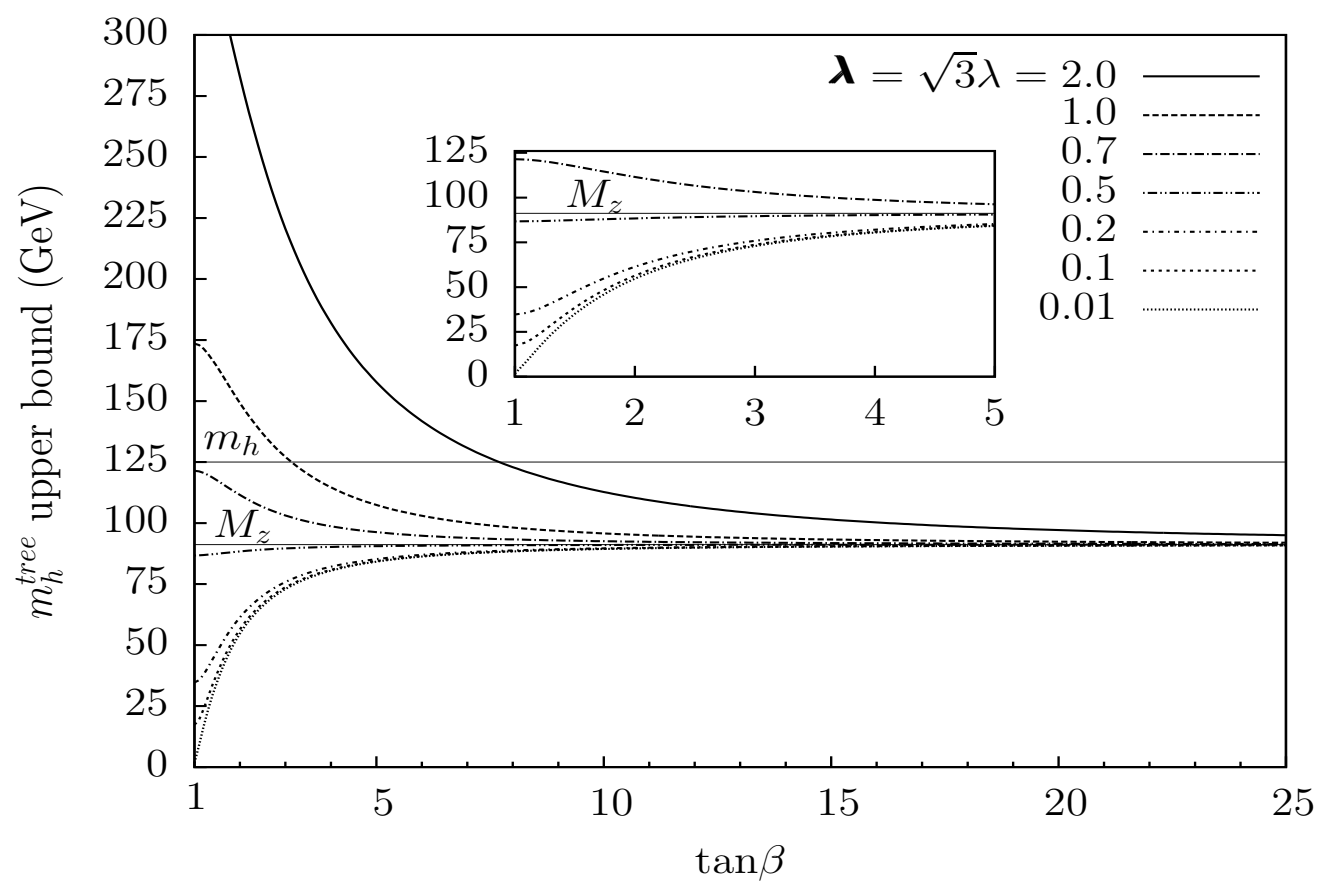

Figure 2. Plot showing the variation of $m_{h}^{\text {tree }}$ upper bound with $\tan \beta$ for different $\lambda(\equiv \sqrt{3} \lambda)$ values as calculated using eq. (4.2). Here $m_{h}$ and $M_{Z}$ represent the experimentally measured mass of the Higgs and $Z$ boson, i.e. $125 \mathrm{GeV}$ and $91.187 \mathrm{GeV}$, respectively. The region of $\tan \beta \leq 5$ has been zoomed for $\boldsymbol{\lambda} \leq 0.7$ values for the convenience of representation.

In the case of the MSSM the $2^{\text {nd }}$ term of eq. (4.2) is absent. Hence, the maximum possible tree-level mass is about $M_{Z}$ as $\tan \beta \gg 1$ and consequently a contribution as large as 0.38 times of the tree-level mass from other sources (for example through the loops) is essential to reach the target of $125 \mathrm{GeV}$. The necessity of a larger contribution over the tree-level mass to reach the target of $125 \mathrm{GeV}$ grows as $\tan \beta$ takes moderate to small values. For example, with $\tan \beta=2$, eq. (4.2) predicts the upper bound of $m_{h}^{\text {tree }}$ about $55 \mathrm{GeV}$. Hence, to reach $125 \mathrm{GeV}$ one needs a contribution which is at least $\approx 1.3$ times larger compared to the $m_{h}^{\text {tree }}$.

On the contrary, as has already been mentioned in ref. [23], in the $\mu \nu \mathrm{SSM}$ one can reach $125 \mathrm{GeV}$ solely with the tree-level contribution. One can observe from eq. (4.2) that $m_{h}^{\text {tree }}$ enhances with an increase in $\boldsymbol{\lambda}$. Thus, even at the limit $\tan \beta \rightarrow 1, m_{h}^{\text {tree }} \neq 0$. The variation of the tree-level mass, $m_{h}^{\text {tree }}$ as calculated using eq. (4.2), with a change in $\tan \beta$ values for the different fixed values of $\boldsymbol{\lambda}$ is shown in figure 2. Here $\tan \beta$ values greater than 25 have been intentionally truncated since $m_{h}^{\text {tree }}$ practically saturates around $M_{Z}$ for $\tan \beta>25$ with a mild exception for $\boldsymbol{\lambda}=2$. It is also worth noticing that a class of flavour observables (e.g., $B_{s}^{0} \rightarrow \mu^{+} \mu^{-}$), as already stated, depending on the other parameters posses high power sensitivity to $\tan \beta$ which in turn can put strong constraints on the large $\tan \beta$ values.

In order to discuss figure 2 , let us choose three regions in $\boldsymbol{\lambda}$ values, namely (a) small to moderate, i.e. $\boldsymbol{\lambda} \lesssim 0.01$ to $\boldsymbol{\lambda} \leq 0.1$, (b) moderate to large, i.e. $\boldsymbol{\lambda}>0.1$ to $\boldsymbol{\lambda} \leq 0.7$, which 
is the maximum possible value of $\boldsymbol{\lambda}$ maintaining its perturbative nature up to the scale of a grand unified theory (GUT) $\left(\sim 10^{16} \mathrm{GeV}\right)$, and finally (c) dominant, $\boldsymbol{\lambda}>0.7$. These ranges will also be useful later when we continue our discussion in section 5 and section 6 .

(a) Small to moderate $\lambda$ : in this range of $\boldsymbol{\lambda}$ values the quantity $\boldsymbol{\lambda}^{2}$ takes values from $\sim 10^{-4}$ to $10^{-2}$. Hence the maximum value of $m_{h}^{\text {tree }}$ using eq. (4.2) with $\boldsymbol{\lambda}=0.1$ goes as $\approx 56.5 \mathrm{GeV}$ for $\tan \beta=2$ which is $\approx 1 \mathrm{GeV}$ more compared to a similar situation in the MSSM. Keeping all the other parameters fixed, $m_{h}^{\text {tree }}$ is estimated as $\approx 55 \mathrm{GeV}$ for $\boldsymbol{\lambda}=0.01$ as well as for $\boldsymbol{\lambda}=0$. The real difference is only $\sim 0.02 \mathrm{GeV}$ when $\boldsymbol{\lambda}$ changes from 0 to 0.01 . Since this change is rather insignificant, we do not explicitly show $\boldsymbol{\lambda}=0$ (MSSM-like) scenario in figure 2. With larger $\tan \beta$ values (say 10 or more) this extra contribution diminishes and $m_{h}^{\text {tree }} \rightarrow M_{Z}$ as $\tan \beta \gg 1$. This feature is also apparent from figure 2. It is thus essential to have additional contributions to raise $m_{h}^{\text {tree }}$ up to $125 \mathrm{GeV}$, as has been measured experimentally.

A possible source of extra tree-level mass can also arise through the mixing of doubletlike states with other states like the left- and the right-handed sneutrinos. The mixing between the doublet-like states with the left-handed sneutrinos, however, has negligible effect on the tree-level Higgs mass as the concerned terms are suppressed through very small $Y_{\nu_{i j}}$ and $\nu_{i}[23,29]$. On the other hand, the mixing between the doublet-like and the right-handed sneutrino-like states appears through $\lambda_{i}$, which are usually several orders of magnitude larger compared to $Y_{\nu_{i j}}$. These mixing can raise the tree-level lightest doublet-like Higgs mass in the case when the right-handed sneutrino-like states are lighter compared to the lightest doublet-like Higgs. Note that the parameters $\kappa$ and $A_{\kappa}$ are the key ingredients to determine the mass scale of these right-handed sneutrino states [23, 29]. In this situation, the lightest doublet-like state feels a push away effect from the lighter singlet-like states which can contribute to push $m_{h}^{\text {tree }}$ (as estimated using eq. (4.2)) a bit further towards $125 \mathrm{GeV}$. Unfortunately, for this range of $\boldsymbol{\lambda}$ values the push-up effect is normally small owing to the small singlet-doublet mixing which is driven by $\boldsymbol{\lambda}[23,49]$.

One can also get heavy singlet-like states with the other choices of $\kappa, A_{\kappa}$. This scenario, however, has the opposite effect on the doublet-like lightest state, namely to lower the mass.

Necessity for an additional contribution is now apparent for this corner of the parameter space to accommodate a $125 \mathrm{GeV}$ doublet-like Higgs. This time the contribution is coming from a well known source, namely the loop effects [189-207]. For $\tan \beta \lesssim 5$, a loop contribution as large as the tree-level mass (e.g., $m_{h}^{\text {tree }} \approx 56 \mathrm{GeV}$ for $\tan \beta=2$ and $\boldsymbol{\lambda}=0.1$ ) is required. Thus, in this region of the parameter space the issue of accommodating a $125 \mathrm{GeV}$ Higgs is practically similar to that of the MSSM, where large masses for the third-generation squarks and/or large trilinear soft-SUSY breaking terms are essential [208-210], without which a $125 \mathrm{GeV}$ Higgs mass is hardly attainable. The smallest A-terms and the average squark masses can be (with $\tan \beta>20$ ) around $1000 \mathrm{GeV}$ and $500 \mathrm{GeV}$, respectively. A small A-terms is possible only by decoupling the scalars to at least $5 \mathrm{TeV}$ [210]. A light third generation squark, especially a stop, on the other hand, is natural in the so-called maximal mixing scenario [195]. These issues indicate that the novel signatures from the SUSY particles (e.g., from a light stop or sbottom) are less generic in 
this region of $\boldsymbol{\lambda}$. Nevertheless, novel differences are feasible for Higgs decay phenomenology, especially in the presence of singlet-like lighter states which has already been discussed in section 3 .

Let us finally note that the effects of loop contributions are normally negligible for the singlet states, however, when $\kappa \sim 0.1$ or larger, the singlet states can receive a large loop correction $\propto \kappa^{2}$. This happens when the singlet-like states are heavier compared to the lightest doublet-like state.

(b) Moderate to large $\lambda$ : for this range of $\boldsymbol{\lambda}$ values $(>0.1$ to $\leq 0.7)$ the maximum of $m_{h}^{\text {tree }}$ can go beyond $M_{Z}$, especially for $\tan \beta \lesssim 5$ and $\boldsymbol{\lambda} \sim 0.7$. This is also clear from figure 2 . In fact, depending on $\boldsymbol{\lambda}$, in this region the maximum of $m_{h}^{\text {tree }}$ can remain close to the $125 \mathrm{GeV}$ target. For example with $\boldsymbol{\lambda}=0.7, \tan \beta=2(5)$ gives $m_{h}^{\text {tree }} \sim 112(96) \mathrm{GeV}$ using eq. (4.2). This is $\approx 100 \%(14 \%)$ enhancement compared to the MSSM scenario with the same $\tan \beta$. Thus, one needs $\sim 12 \%$ (30\%) contribution from other sources to reach the $125 \mathrm{GeV}$ milestone. The necessity of larger (compared to the given numbers) additional contribution emerges as $\boldsymbol{\lambda}$ picks up smaller values, say 0.2 . In this case with $\tan \beta=2$ (5), $m_{h}^{\text {tree }}$ is estimated as $\sim 61(85) \mathrm{GeV}$ and one needs rather large, $\sim 100 \%(47 \%)$ contribution over the tree-level mass to achieve $125 \mathrm{GeV}$. With an intermediate value, say $\boldsymbol{\lambda}=0.5$, $\tan \beta=2(5)$ gives $m_{h}^{\text {tree }}$ as $\sim 88(90) \mathrm{GeV}$ and thus, $\sim 40 \%$ extra contribution over the $m_{h}^{\text {tree }}$ is needed. It is interesting to see from the last calculation and also from figure 2 that the $\boldsymbol{\lambda}=0.5$ line is almost overlapping to the $M_{Z}$ line and consequently magnitude of $m_{h}^{\text {tree }}$ or the amount of extra contribution to reach $125 \mathrm{GeV}$ remains practically the same for all $\tan \beta$ values. Being quantitative, as $\tan \beta$ changes from 2 to 10 , the requirement of an extra contribution over the $m_{h}^{\text {tree }}$ to reach the goal of $125 \mathrm{GeV}$ changes by an amount of $\sim 4 \%$. One should note that $\boldsymbol{\lambda}=0.2$ and 0.7 are translated as $\lambda \sim 0.12$ and $\sim 0.4$, assigning a universality for $\lambda_{i}$.

For this region of $\boldsymbol{\lambda}$, the singlet-doublet mixing is no longer negligible, particularly as $\boldsymbol{\lambda} \rightarrow$ 0.7. Thus, a state lighter than $125 \mathrm{GeV}$ with the leading singlet composition appears rather difficult without a certain degree of tuning of the other parameters, e.g. $\kappa, \nu^{c}, A_{\kappa}$, $A_{\lambda}$ etc. These issues will be addressed thoroughly later in section 5 . In this situation the extra contribution to $m_{h}^{\text {tree }}$ is favourable through a push-up action from the singlet states compared to small to moderate $\boldsymbol{\lambda}$ scenario. However, a sizable doublet impurity makes it rather hard for these states to escape the collider constraints. The situation is a bit ameliorated with smaller $\boldsymbol{\lambda}$, say around 0.2 or 0.3 .

Once again a contribution from the loops is needed to reach the $125 \mathrm{GeV}$ target. However, depending on the values of $\boldsymbol{\lambda}$ and $\tan \beta$ the requirement sometime is much softer compared to small to moderate $\boldsymbol{\lambda}$ scenario. Beyond $\tan \beta=10$, at least $\sim 35 \%$ of the treelevel contribution from the other sources is required to reach $125 \mathrm{GeV}$ even with $\boldsymbol{\lambda}=0.7$, which is $\sim 5 \%$ small compared to a similar scenario with $\boldsymbol{\lambda}=0.1$. Considering the same analysis for $\tan \beta=3$ one gets $\sim 48 \%$ difference between $\boldsymbol{\lambda}=0.7$ and $\boldsymbol{\lambda}=0.1$ scenarios. Hence, depending on $\tan \beta$ and $\boldsymbol{\lambda}$, the necessity of heavy third-generation squarks and/or large trilinear soft-SUSY breaking term may or may not appear essential for this region [208]. For example, for the scenario studied in ref. [50], where $\tan \beta=3.7$ and 
$\lambda_{i}=0.11$ (i.e., $\boldsymbol{\lambda} \approx 0.2$ ), one needs $A_{t}=2.4 \mathrm{TeV}$ and stop masses about $1 \mathrm{TeV}$. Moving towards $\boldsymbol{\lambda} \sim 0.7$, on the contrary, room for the third-generation squarks lighter than $1 \mathrm{TeV}$ is possible. For example, with $\tan \beta=2$ and $\boldsymbol{\lambda}=0.7$, stop masses and A-terms of about $300 \mathrm{GeV}$ are sufficient to raise the Higgs mass to $125 \mathrm{GeV}$ [208]. It is also worth noticing that the naturalness is therefore improved with respect to the MSSM or smaller values of $\boldsymbol{\lambda}$.

Lighter singlet states, as already stated, are also feasible here with some degree of parameter tuning. Although they lead to unusual signatures at the LHC, however, a sizable doublet component makes it hard for these states to escape a group of the experimental constraints, as mentioned in the introduction.

(c) Dominant $\lambda$ : if one relaxes the idea of perturbativity up to the GUT scale, large values (>0.7) for $\boldsymbol{\lambda}$ emerge naturally. ${ }^{10}$ Assuming a scale of new physics around $10^{11} \mathrm{GeV}$, the perturbative limit on $\boldsymbol{\lambda}$ gives $\boldsymbol{\lambda} \sim 1.0$ (i.e., $\lambda \sim 0.58$ ) [23]. Pushing the scale of new physics further below to $10 \mathrm{TeV}$, this limit gives $\boldsymbol{\lambda} \sim 2$ (i.e., $\lambda \sim 1.1$ ). In this region, as also shown in figure 2, the maximum of $m_{h}^{\text {tree }}$ as evaluated from eq. (4.2) can remain well above $125 \mathrm{GeV}$ even up to $\tan \beta \sim 8$ for $\boldsymbol{\lambda} \sim 2$. For $\boldsymbol{\lambda}=1$, a similar analysis gives $\tan \beta \sim 2$ as the upper limit. Here with $\boldsymbol{\lambda}=1$, the maximum of $m_{h}^{\text {tree }}$ for $\tan \beta=2,5$ and 10 is estimated as $\sim 150 \mathrm{GeV}, 108 \mathrm{GeV}$ and $\sim 96 \mathrm{GeV}$, respectively. With $\boldsymbol{\lambda}=2$ these numbers increase further, for example, $\sim 113 \mathrm{GeV}$ when $\tan \beta=10$. The requirement of an extra contribution to reach the target of $125 \mathrm{GeV}$ is thus, rather small and even negative in this corner of the parameter space unless $\tan \beta$ goes beyond 10 or 15 depending on the values of $\boldsymbol{\lambda}$.

A singlet-like state lighter than $125 \mathrm{GeV}$ is rather difficult in this corner of the parameter space due to the large singlet-doublet mixing. In fact even if one manages to get a scalar lighter than $125 \mathrm{GeV}$ with drastic parameter tuning, a push-up action can produce a sizable effect to push the mass of the lightest doublet-like state beyond $125 \mathrm{GeV}$, especially for $\tan \beta \lesssim 10$ taking $\boldsymbol{\lambda}=2$. Moreover, a huge doublet component makes these light states hardly experimentally acceptable. In this region of the parameter space a heavy singlet-like sector is more favourable which can push $m_{h}^{\text {tree }}$ down towards $125 \mathrm{GeV}$. A set of very heavy singlet-like states, even with non-negligible doublet composition is also experimentally less constrained.

It is needless to mention that the amount of the loop correction is much smaller in this region compared to the two previous scenarios. For example, with $\tan \beta=10$, one needs a loop effect $\sim 11 \%$ and $30 \%$ with $\boldsymbol{\lambda}=2$ and 1, respectively. One should compare this with the maximum value of $\boldsymbol{\lambda}$ keeping perturbative nature up to the GUT scale, i.e. 0.7, where one needs $\sim 35 \%$ contribution over the tree-level mass for $\tan \beta=10$. Following the above discussion for large values of $\boldsymbol{\lambda}$, this region of the parameter space also favours third-generation squarks lighter than $1 \mathrm{TeV}$, which can be produced with enhanced cross sections and can lead to novel signatures of the model at the LHC. Note that the light third generation of squarks is still allowed by the LHC results, see e.g. refs. [20, 21]. This feature can produce new signatures at colliders with $\not R_{p}$ for this region $\boldsymbol{\lambda}$ values, even when the singlet-like states remain heavier, as stated earlier. One should note that for such a

\footnotetext{
${ }^{10} \mathrm{~A}$ similar scenario in the context of the NMSSM has been popularised as $\lambda$-SUSY [211].
} 
large $\boldsymbol{\lambda}$ value, new loop effects from the right-handed sneutrinos with contributions $\propto \boldsymbol{\lambda}^{2}$ can generate an additional enhancement [212].

We end our discussion on the dominant $\boldsymbol{\lambda}$ scenario with a note of caution. It apparently seems that pushing the scale of perturbativity as low as possible is useful to yield larger and larger $\boldsymbol{\lambda}$ ( $>2$ for instance) values. However, $\boldsymbol{\lambda} \sim 3$ indicates the scale of new physics around $1 \mathrm{TeV}$ which appears to be an extinct possibility from the experimental observations since no definite excess over the SM predictions has been observed to date.

The discussion presented so far favours, in order to obtain the light singlet-like states, small to moderate $\boldsymbol{\lambda}$ region where the singlet-doublet mixing is small. Hence in this corner of the parameter space one can easily get the light singlet-like states with suitable choices of $\kappa, A_{\kappa}$ and $\nu^{c}[30,49,50]$. Although a large loop contribution is essential for this region of the parameter space to reach the $125 \mathrm{GeV}$ target, the associated lighter states have notable consequences in the collider phenomenology of the scalar sector, as already stated in section 3. It is now absolutely essential to investigate the behaviour of $S_{i}^{0}, P_{i}^{0}$ and $\widetilde{\chi}_{i+3}^{0}$ masses for these three regions of $\boldsymbol{\lambda}$ values, which is what we plan for the next section.

\section{Masses of the singlet-like states in the $\mu \nu \mathrm{SSM}$}

In this section we first aim to identify the relevant set of parameters which controls the mass scale of the singlet-like scalars, pseudoscalars and neutralinos in the $\mu \nu$ SSM. Subsequently, we present a set of general expressions for the mass terms of the singlet-like $S_{i}^{0}, P_{i}^{0}$ and $\widetilde{\chi}_{4,5,6}^{0}$ states. We further extend our analyses over the three regions of $\boldsymbol{\lambda}$ values, as of the last section, accompanied by a discussion regarding the scale of the other crucial parameters. In this section and from henceforth we use $\widetilde{\chi}_{i+3}^{0}, i=1,2,3$, to denote the three lightest neutralinos in lieu of $\widetilde{\chi}_{4,5,6}^{0}$.

In order to proceed systematically it is crucial to identify first the set of most relevant parameters which controls the tree-level masses and mixing of the electroweak scalar and fermion sectors in the $\mu \nu \mathrm{SSM}$. Considering universal $\nu_{i}^{c}\left(\equiv \nu^{c}\right)$, flavour-diagonal but quasidegenerate $\kappa_{i j k}\left(\equiv \kappa_{i}\right)$ together with the universal and flavour-diagonal $A_{\lambda}$ and $A_{\kappa}$, the parameters that control the electroweak fermions are

$$
M_{1}, M_{2}, \boldsymbol{\lambda}, \kappa_{i}, \nu^{c}, \tan \beta
$$

In the same spirit, the relevant parameters for the scalars (CP-even and odd) are

$$
\lambda, \kappa_{i}, \nu^{c}, \tan \beta, A_{\lambda}, A_{\kappa} .
$$

Note that with our choice of quasi-degenerate $\kappa_{i}$ and universal $A_{\kappa}$, each of the three $S_{i}^{0}, P_{i}^{0}$ and $\tilde{\chi}_{i+3}^{0}$ states are closely spaced in masses. Assumptions for $Y_{\nu_{i j}}$ (chosen to be flavour diagonal), $\nu_{i}$ and $A_{\nu}$ (chosen to be flavour diagonal and universal) are not explicitly mentioned in eqs. (5.1) and (5.2). The left-handed neutrinos and sneutrinos, as already stated, couple to the remaining states through $Y_{\nu_{i j}}$ or $\nu_{i}[22,23,29]$. Both of these $\left(Y_{\nu_{i j}}, \nu_{i}\right)$ are constrained to be small $\left(\mathcal{O}\left(10^{-6}-10^{-7}\right), \mathcal{O}\left(10^{-4}-10^{-5}\right)\right.$, respectively $\left.[22,23,29-32]\right)$, in order to accommodate the measured neutrino data [35-37] with a electroweak scale 
seesaw mechanism $[22,23,29,30,33,34]$. Hence, the admixture of these states does not produce any significant changes in the phenomenological analyses considered here and thus, are not shown in eqs. (5.1) and (5.2).

It has already been emphasised that we are looking for the hints of new physics with $S_{4}^{0} \rightarrow X X$ decay modes with $X$ as the light singlet-like $S_{i}^{0}, P_{i}^{0}, \widetilde{\chi}_{i+3}^{0}$. The mass scales of these states, as shown in refs. [23, 29] depend on the set of parameters shown in eqs. (5.1) and (5.2). We work in the region of low $\tan \beta$ to avoid a class of flavour physics constraints, e.g. $B_{s}^{0} \rightarrow \mu^{+} \mu^{-}$. Further, we assume a higgsino-like $\widetilde{\chi}_{4}^{ \pm}$and $\mu \gtrsim 100 \mathrm{GeV}$, consistent with the LEP lighter chargino mass bound [172]. One advantage of this choice is that one can push $M_{2}$ to proper values such that $m_{\widetilde{g}} \gtrsim 1.2 \mathrm{TeV}[20,21]$ appears naturally without spoiling the gaugino universality at the GUT scale. On the dark side, depending on the value of $\boldsymbol{\lambda}(\sqrt{3} \lambda)$, a singlino-like neutralino with mass $\lesssim M_{Z} / 2$ may posses sizable higgsino admixture (remember $5^{\text {th }}$ term of eq. (2.1)) and thereby gets severely constrained from the measured $Z$ decay width [172]. Of course, one can live with a light $(\sim \mathcal{O}(100 \mathrm{GeV}))$ gauginolike $\widetilde{\chi}_{4}^{ \pm}$without the gaugino universality relation for $M_{3}$ yet maintaining ${ }^{11} M_{2}=2 M_{1}$. In this case $\widetilde{\chi}_{7}^{0}$ is bino-like and can coexist with the measured $Z$ decay width [172] even being lighter than $M_{Z} / 2$, since a tree-level $Z$-bino-bino coupling does not exist. We, however, do not consider this possibility in order to work with a minimal number of the free parameters.

Summarising, the parameters relevant for this analysis are

$$
\lambda, \kappa_{i}, \nu^{c}, \tan \beta, M_{1}, A_{\lambda} \text { and } A_{\kappa} .
$$

It is clear from the mass matrices $[23,29]$ that $\kappa_{i}$ and $A_{\kappa}$ are the two crucial parameters to determine the masses of the singlet states, originating from the self-interactions. The remaining parameters $\boldsymbol{\lambda}$ (through $\lambda$ ) and $A_{\lambda}$ not only appear in the said interactions, but also control the mixing between the singlet and the doublet states and hence, contribute in determining the mass scale. In the limit of a vanishingly small $\boldsymbol{\lambda}$, the singlet states are completely decoupled from the doublets. ${ }^{12}$ It is thus apparent that $\boldsymbol{\lambda}$ is undoubtedly the most relevant parameter for this analysis. Another aspect of the parameter $\boldsymbol{\lambda}$, i.e. to yield additional contribution to the tree-level lightest doublet-like Higgs mass has already been discussed in the previous section.

In order to proceed further, we continue with the three regions of $\boldsymbol{\lambda}$ values as already introduced in the last section. Similar ranges of $\boldsymbol{\lambda}$ values, but in the context of SUSY signatures for the NMSSM has been mentioned in ref. [213]. For each of these three $\boldsymbol{\lambda}$ zones, we will address in section 6 the phenomenological signatures from the new $S_{4}^{0}$ decays, including effects coming from the variations of $\kappa_{i}, A_{\kappa}, \tan \beta$ and $A_{\lambda}$ parameters.

In order to give a better interpretation of these scenarios, we start with the approximate analytical formulae for $m_{S_{i}^{0}}^{2}, m_{P_{i}^{0}}^{2}$ and $m_{\widetilde{\chi}_{i+3}^{0}}$. A set of expressions for these masses

\footnotetext{
${ }^{11}$ If one considers a heavy gaugino-like $\widetilde{\chi}_{4}^{ \pm}$, for example with $M_{2} \sim 400 \mathrm{GeV}$, the gaugino universality appears naturally with $m_{\widetilde{g}} \gtrsim 1.2 \mathrm{TeV}$. The scenario with a heavy higgsino-like $\widetilde{\chi}_{4}^{ \pm}$is somewhat inconsistent with the idea of naturalness. The breaking of universality relation between $M_{1}, M_{2}$ will also increase the number of free parameters further.

${ }^{12}$ One should simultaneously consider a very large $\nu^{c}$ such that $\mu\left(\sqrt{3} \boldsymbol{\lambda} \nu^{c}\right)$ remains $\gtrsim 100 \mathrm{GeV}$, as required by the LEP lighter chargino mass bound.
} 
with three families of the right-handed neutrino superfields using a simplified parameter choice (see eqs. (5.1) and (5.2)), even in the region of small to moderate $\boldsymbol{\lambda}$, appears rather complicated due to the index structure of the parameters $\kappa_{i} \mathrm{~s}$. The expressions for the mass terms are relatively simpler for $P_{i}^{0}$ and $\chi_{i+3}^{0}$ in the limit of a complete degeneracy in all the relevant parameters, i.e. when eq. (5.3) is rewritten as

$$
\boldsymbol{\lambda}(\equiv \sqrt{3} \lambda), \kappa, \mu, \tan \beta, M_{1}, A_{\lambda}, A_{\kappa},
$$

where we have replaced the $\nu^{c}$ parameter of eq. (5.3) with the $\mu$ parameter $\equiv \sqrt{3} \lambda \nu^{c}$. Note that, even with the assumptions of eq. (5.4), the expressions for the squared mass terms remain rather complicated for the scalars $S_{i}^{0}$. In order to investigate the mass terms for $S_{i}^{0}, P_{i}^{0}, \widetilde{\chi}_{i+3}^{0}$ states in more detail in the light of the relevant parameters, as given by eq. (5.4), we start our discussion with $S_{i}^{0}$ and $P_{i}^{0}$ and later we continue with $\widetilde{\chi}_{i+3}^{0}$.

Being illustrative, in the $\mu \nu \mathrm{SSM}$ with the three families of $\hat{\nu}_{i}^{c}$, dimensions of the scalar, pseudoscalar and neutralino mass matrices are $8 \times 8,8 \times 8$ and $10 \times 10$, respectively [23, 29]. Now, as already stated in section 4 , the left-handed sneutrinos couple with the remaining states (i.e., doublet Higgses and the right-handed sneutrinos) through $Y_{\nu_{i j}}$ and $\nu_{i}$. Both of these are constrained to be tiny, as required by a electroweak-scale seesaw mechanism $[22$, 23, 29-31]. Hence, for all practical purposes, the effect of these mixing are negligible on the remaining $5 \times 5$ scalar and pseudoscalar mass matrices. Each of these $5 \times 5$ matrices contains a $2 \times 2$ MSSM-like block (top-left [23, 29]), a $3 \times 3$ block (bottom-right [23, 29]) with the right-handed sneutrino mass terms and finally two $2 \times 3,3 \times 2$ off-diagonal blocks that contain the mixing between the right-handed sneutrinos and the doublet Higgses. Note that the scalar, pseudoscalar and neutralino mass matrices in the $\mu \nu \mathrm{SSM}$ are symmetric $[23,29]$.

Concentrating on the $5 \times 5$ block, as mentioned above, the $3 \times 3$ right-handed sneutrino block, both for the scalar and the pseudoscalar mass matrices, in the light of eq. (5.4) symbolically can be written as $A I_{3 \times 3}+B(\mathcal{I}-I)_{3 \times 3}$. Here $I_{3 \times 3}$ is a $3 \times 3$ identity matrix while $\mathcal{I}_{3 \times 3}$ is a $3 \times 3$ matrix with 1 in all the nine places, $A$ and $B$ are functions of $\boldsymbol{\lambda}, \kappa, \tan \beta$, $A_{\lambda}, A_{\kappa}$ and $\nu^{c}$.

At this stage it is possible to apply a $3 \times 3$ rotation matrix, ${ }^{13}$ constructed with its eigenvectors, ${ }^{14}$ to obtain a $3 \times 3$ rotated right-handed sneutrino mass matrix with non-zero entries, $A-B, A-B$ and $A+2 B$ only in the diagonals. For the pseudoscalars, one also needs to apply a $2 \times 2$ rotation matrix ${ }^{15}$ constructed out of $\sin \beta, \cos \beta\left(\sin \beta=\frac{v_{u}}{v}, \cos \beta=\frac{v_{d}}{v}\right)$, to rotate away the would be Goldstone boson.

With this simple operation, two of the entries of the rotated right-handed sneutrino mass matrix, both for the scalars and the pseudoscalars, are exactly degenerate in masses and are completely separated from the rest of the mass matrix. In other words, after the aforementioned $3 \times 3$ rotation, two of the three eigenvalues of the right-handed sneutrino

\footnotetext{
${ }^{13}$ Note that the actual rotation matrix must be $5 \times 5$ in size, however, has a $2 \times 2$ identity matrix in the top-left $2 \times 2$ block and zeros in the off-diagonal $2 \times 3$ block.

${ }^{14}$ One needs to use Gram-Schmidt procedure to obtain a proper orthonormal set of eigenvectors since two of the eigenvalues of $A I_{3 \times 3}+B(\mathcal{I}-I)_{3 \times 3}$ matrix are identical.

${ }^{15}$ Again the actual one is $5 \times 5$ in size with a $I_{3 \times 3}$ for the right-handed sneutrino block and zeros in the $2 \times 3$ off-diagonal block.
} 
mass matrix get decoupled and remain as the pure singlet-like states without any doublet contamination. The third eigenvalue, namely the one which goes as $A+2 B$, however, mixes with the doublet-like states and eventually appears with a much complicated form.

In the case of the pseudoscalar, after rotating away the Goldstone boson, the remaining matrix is a simple $2 \times 2$ matrix and thus, it is possible to extract the exact modified (i.e., after mixing with the doublets) formula for that $A+2 B$ eigenvalue.

The absence of Goldstone mode for the scalars, on the other hand, leaves the resultant mass matrix $3 \times 3$ in size after separating out the two degenerate eigenvalues. Hence, it is rather difficult to obtain a simple analytical formula for the scalar right-handed sneutrino that mixes with the doublet Higgses. A naive attempt to extract this eigenvalue using the idea of $x_{l} \approx \operatorname{det}\left[\operatorname{Mat}_{n \times n}\right] / \operatorname{det}\left[\operatorname{Mat}_{(n-1) \times(n-1)}\right]\left(x_{l}\right.$ represents the lightest eigenvalue of a $n \times n$ matrix ' $M a t$ ') fails since the resultant expression contains terms up to $\lambda^{5}$ (the parameter which controls the singlet-doublet admixing, see eqs. (2.1) and (2.2)) with nonnegligible coefficients in front.

A note of caution must be emphasised here, i.e. with the choice of $\kappa_{i j k}=\kappa \delta_{i j} \delta_{j k}$, two of the eigenvalues of the scalar and the pseudoscalar squared mass matrices appear degenerate in masses with no doublet impurity. These states, when appear in the bottom of the mass spectrum, are highly stable in nature. ${ }^{16}$ This artificial stability can be broken by introducing mild splittings in $\kappa_{i}$ values $[49,50]$. Their composition can, nevertheless, still remain dominantly singlet-like depending on the values of the other parameters.

We have further verified that our approximate analytical formulae agree rather well with a full numerical evaluation. In the limit of mild non-degeneracy in $\kappa_{i} \mathrm{~s}$, all three singlet-like states adhere doublet impurity, however, the amount of doublet component is small for the aforesaid two degenerate states which are now mildly separated in masses [50].

Turning towards the neutralinos, one can think of a similar rotation to the $7 \times 7$ block that contains a $4 \times 4$ MSSM-like block (top-left [23, 29]), a $3 \times 3$ block (bottomright $[23,29])$ with the right-handed neutrino Majorana mass terms and two off-diagonal $4 \times 3$ and $3 \times 4$ blocks that contain the mixing terms between the MSSM-like neutralinos and the right-handed neutrinos. For this propose, we construct a set of the three new orthonormal eigenvectors using linear combination of the three existing trivial orthonormal eigenvectors, ${ }^{17}$ arising from the diagonal $3 \times 3$ right-handed neutrino mass matrix. This mathematical operation, just like the case of the $S_{i}^{0}$ and $P_{i}^{0}$, decouples out the mass terms for the two right-handed neutrinos from the rest of the mass matrix, while the third one mixes with the other MSSM-like neutralinos and has an intricate expression for the mass term.

\footnotetext{
${ }^{16}$ The stability is not absolute as we have neglected the tiny but non-vanishing contributions from the terms involving $Y_{\nu}$ or $\nu_{i}$. A similar construction of the NMSSM with multiple singlets will give absolute stability to the set of lightest degenerate states.

${ }^{17}$ The original eigenvectors are $(1,0,0),(0,1,0)$ and $(0,0,1)$ while the modified ones are $\frac{1}{\sqrt{3}}(1,1,1)$, $\frac{1}{\sqrt{2}}(1,0,-1)$ and $\frac{1}{\sqrt{6}}(1,-2,1)$. These new ones are also used for the rotation of the scalar and pseudoscalar mass matrices. From the structure of these eigenvectors it is clear that mathematically we are rotating the initial right-handed sneutrino/neutrino basis to a specific basis where one of the combinations is completely symmetric (eigenvector $\frac{1}{\sqrt{3}}(1,1,1)$ ) and mixes with the other states while the remaining two combinations are antisymmetric and remain decoupled from the other states.
} 
Now we are in a stage to write down the analytical expressions for the mass terms of the three singlet or right-handed neutrino, sneutrino-like $\widetilde{\chi}_{i+3}^{0}, P_{i}^{0}$ and $S_{i}^{0}$ states as

$$
\begin{aligned}
m_{\widetilde{\chi}_{1+3,2+3}^{0}} & \equiv m_{\widetilde{\chi}_{U_{1,2}^{0}}^{0}}=2 \kappa \nu^{c}, \\
m_{\widetilde{\chi}_{3+3}^{0}} & \equiv m_{\widetilde{\chi}_{M}^{0}}=2 \kappa \nu^{c}+\frac{1}{6} \frac{\lambda^{2} v^{2}}{\mu}\left(\frac{1}{f(T)}-\frac{4 \mathcal{M} \mu}{v^{2}}\right)\left(1-\frac{\mathcal{M} \mu}{v^{2} f(T)}\right)^{-1}, \\
m_{P_{1,2}^{0}}^{2} & \equiv m_{P_{U_{1,2}}^{0}}^{2}=-3 \kappa A_{k} \nu^{c}+\left(\frac{A_{\lambda}}{\mu}+\frac{4}{\sqrt{3}} \frac{\kappa}{\lambda}\right) f(T) \boldsymbol{\lambda}^{2} v^{2}-\lambda^{2} v^{2}, \\
m_{P_{3}^{0}}^{2} & \equiv m_{P_{M}^{0}}^{2}=-3 \kappa A_{k} \nu^{c}+\frac{A_{\lambda}}{A_{\lambda}+\kappa \nu^{c}} 3 \sqrt{3} f(T) \boldsymbol{\lambda} \kappa v^{2}, \\
m_{S_{1,2}^{0}}^{2} & \equiv m_{S_{U_{1,2}}^{0}}^{2}=\kappa A_{k} \nu^{c}+4 \kappa^{2} \nu^{c 2}+\frac{A_{\lambda}}{\mu} f(T) \boldsymbol{\lambda}^{2} v^{2}-\boldsymbol{\lambda}^{2} v^{2}, \\
m_{S_{3}^{0}}^{2} & \equiv m_{S_{M}^{0}}^{2} \\
& =\frac{a_{0}+a_{1} f(T) \boldsymbol{\lambda}+4 a_{2} f(T)^{2} \boldsymbol{\lambda}^{2}+24 a_{3} f(T) \boldsymbol{\lambda}^{3}-24 a_{4} \boldsymbol{\lambda}^{4}+864 a_{5} f(T)^{3} \boldsymbol{\lambda}^{5}}{b_{0}+4 b_{1} f(T)^{3} \boldsymbol{\lambda}+24 b_{2} f(T)^{2} \boldsymbol{\lambda}^{2}-24 b_{3} f(T)^{3} \boldsymbol{\lambda}^{3}}
\end{aligned}
$$

where $a_{0, \ldots, 5}$ and $b_{0, \ldots, 3}$, in the expression of $m_{S_{M}^{0}}^{2}$ are complicated functions of the model parameters and are given by

$$
\begin{aligned}
a_{0}= & G_{2} \kappa v^{4} \nu^{c 3}\left(A_{\lambda}+\kappa \nu^{c}\right)\left(A_{\kappa}+4 \kappa \nu^{c}\right) g(T)^{2}, \\
\sqrt{3} a_{1}= & -G_{2} v^{2} \nu^{c}\left\{4 A_{\lambda}^{2} f(T)^{2} v^{4}+A_{\lambda}\left(2 v_{d}^{2}+v^{2}\right)\left(v^{2}+2 v_{u}^{2}\right) \kappa \nu^{c}\right. \\
& \left.+4 \kappa \nu^{c} v^{4}\left(\kappa \nu^{c} g(T)^{2}-A_{\kappa} f(T)^{2}\right)\right\} f(T), \\
3 a_{2}= & v^{4}\left\{G_{2} A_{\lambda} v^{4} f(T)^{2}+6 A_{\lambda}\left(G_{2} v^{2}-A_{\lambda}^{2}\right) \nu^{c 2}+6 \kappa\left(A_{\lambda}\left(A_{\kappa}-5 A_{\lambda}\right)+2 G_{2} v^{2}\right) \nu^{c 3}\right. \\
& \left.+6 \kappa^{2}\left(A_{\kappa}-4 A_{\lambda}\right) \nu^{c 4}\right\} f(T)^{2}, \\
3 \sqrt{3} a_{3}= & v^{2} \nu^{c}\left\{-6 G_{2} v^{4} \nu^{c 2} f(T)^{2}+2 A_{\lambda}^{2}\left(v^{4} f(T)^{2}+3 v^{2} \nu^{c 2}\right)\right. \\
& \left.+\kappa \nu^{c}\left(\left(5 A_{\lambda}-A_{\kappa}\right) v^{4} f(T)^{2}+18 A_{\lambda} v^{2} \nu^{c 2}\right)+12 \kappa^{2} v^{2} \nu^{c 4}\right\} f(T), \\
9 a_{4}= & A_{\lambda} v^{4}\left(v^{2} f(T)^{2}+3 \nu^{c 2}\right)^{2}+3 \kappa v^{2} \nu^{c 3}\left(4 v^{4} f(T)^{2}+3 v^{2} \nu^{c 2}\right), 9 \sqrt{3} a_{5}=v^{6} \nu^{c 3} f(T)^{3}, \\
b_{0}= & G_{2} v^{4} \nu^{c 2}\left(A_{\lambda}+\kappa \nu^{c}\right) g(T)^{2}, \sqrt{3} b_{1}=G_{2} v^{6} \nu^{c} f(T)^{3}, \\
3 b_{2}= & v^{4} \nu^{c 2}\left(A_{\lambda}+\kappa \nu^{c}\right) f(T)^{2}, 3 \sqrt{3} b_{3}=v^{6} \nu^{c} f(T)^{3} .
\end{aligned}
$$

Here we have used $\sqrt{3} \lambda=\lambda, v=\frac{v_{u}}{\sin \beta}=\frac{v_{d}}{\cos \beta}=\sqrt{v_{u}^{2}+v_{d}^{2}}, G_{2}=g_{1}^{2}+g_{2}^{2}, T=\tan \beta$ and $\mathcal{M}=M_{1} M_{2} /\left(g_{1}^{2} M_{2}+g_{2}^{2} M_{1}\right)$ with $g_{1}\left(g_{2}\right)$ as the $\mathrm{U}(1)(\mathrm{SU}(2))$ gauge coupling. The functions $f(T)=\frac{T}{1+T^{2}}$ and $g(T)=\frac{1-T^{2}}{1+T^{2}}$ are derived using $v=\frac{v_{u}}{\sin \beta}=\frac{v_{d}}{\cos \beta}$, and finally we use $\mu=3 \lambda \nu^{c} \equiv \sqrt{3} \lambda \nu^{c}$. Subscripts ' $\mathrm{U}$ ' and ' $\mathrm{M}$ ' are used to interpret the nature of the concerned state, i.e. whether it remains an 'U'nmixed singlet-like without a doublet contamination or appears as a 'M'ixed one with non-vanishing doublet composition.

With these formulas ready we are now in a state to investigate the behaviour of $m_{\widetilde{\chi}_{i+3}^{0}}$, $m_{P_{i}^{0}}^{2}$ and $m_{S_{i}^{0}}^{2}$ for the three different ranges of $\boldsymbol{\lambda}$ values, as already introduced in the last section, along with the necessary discussion about the other crucial parameters. Before 
we proceed further, it will be useful to reevaluate eq. (5.5) in the limit of $\tan \beta \rightarrow \infty$ (i.e. $f(T) \rightarrow 0$ and $g(T)^{2} \rightarrow 1$ ) when the formulae take simpler forms as

$$
\begin{aligned}
& m_{\widetilde{\chi}_{U_{1,2}}^{0}}=2 \kappa \nu^{c}, m_{\widetilde{\chi}_{M}^{0}} \approx 2 \kappa \nu^{c}-\frac{1}{6} \frac{\lambda^{2} v^{4}}{\mathcal{M} \mu^{2}}, \\
& m_{P_{U_{1,2}}^{0}}^{2} \approx-3 \kappa A_{k} \nu^{c}-\lambda^{2} v^{2}, m_{P_{M}^{0}}^{2} \approx-3 \kappa A_{k} \nu^{c}, \\
& m_{S_{U_{1,2}}^{0}}^{2} \approx \kappa A_{k} \nu^{c}+4 \kappa^{2} \nu^{c 2}-\lambda^{2} v^{2}, m_{S_{M}^{0}}^{2} \approx \kappa A_{k} \nu^{c}+4 \kappa^{2} \nu^{c 2}-\frac{8 \mu^{2}}{g_{1}^{2}+g_{2}^{2}} \lambda^{2},
\end{aligned}
$$

where co-efficient of the $\lambda^{2}$ term in the expression of $m_{S_{M}^{0}}^{2}$ is estimated using $\mu=\sqrt{3} \lambda \nu^{c}$.

It is evident from eq. (5.7) that unless $\boldsymbol{\lambda}$ is small to moderate (i.e., $0.01 \lesssim \boldsymbol{\lambda} \leq 0.1$ ) it is in general hard to accommodate a complete non-tachyonic light spectrum (i.e. $\lesssim m_{S_{4}^{0}} / 2$ ) for both the scalars and pseudoscalars in the limit of large $\tan \beta$ without a parameter tuning. A non-tachyonic $\widetilde{\chi}_{M}^{0}$, on the other hand, is possible up to $\boldsymbol{\lambda} \sim 0.7$ unless $2 \kappa \nu^{c} \lesssim 10 \mathrm{GeV}$ or $\mathcal{M} \ll \mathcal{O}(v, \mu)$ in the limit of relaxing the gaugino universality condition at the high energy scale. ${ }^{18}$ The limit $\boldsymbol{\lambda} \rightarrow 0$ (with $\mu \gtrsim 100 \mathrm{GeV}$ as required from the lighter chargino mass bound) together with a proper choice of the other relevant parameters (i.e., $\kappa, A_{\kappa}, \nu^{c}$ ) assures the light singlet-like $\widetilde{\chi}_{i+3}^{0}, P_{i}^{0}, S_{i}^{0}$ states in the mass spectrum with a vanishingly small doublet composition. We emphasise here that although the expressions for $\widetilde{\chi}_{i+3}^{0}, S_{i}^{0}$ and $P_{i}^{0}$ mass terms as shown by eq. (5.7) are much simpler compared to the same as given by eq. (5.5), this region of the parameter space with $\tan \beta \gg 1$ is severely constrained from diverse experimental results. This is because the branching fractions for some low-energy processes (e.g. $B_{s}^{0} \rightarrow \mu^{+} \mu^{-}$), as discussed before in sections 3 and 4 , depending on the other relevant parameters are sensitive to the high powers of $\tan \beta$ and thus, can produce large branching ratios for these processes in an experimentally unacceptable way in the limit of $\tan \beta \gg 1$. For this reason, we will not explicitly address the behaviour of $m_{\widetilde{\chi}_{i+3}^{0}}, m_{P_{i}^{0}}, m_{S_{i}^{0}}$ for various ranges of $\boldsymbol{\lambda}$ values in this limit.

The other limit, i.e. small $\tan \beta$, on the contrary, is useful from the view point of raising the mass of the lightest doublet-like scalar (see eq. (4.2)) towards $125 \mathrm{GeV}$, especially for moderate to large $\boldsymbol{\lambda}$ values as already addressed in section 4 . However, as shown by eq. (5.5), not all the mass formulas for the light $\widetilde{\chi}_{i+3}^{0}, P_{i}^{0}, S_{i}^{0}$ are simple structured in this region.

In order to understand the behaviour of $m_{\widetilde{\chi}_{i+3}^{0}}, m_{P_{i}^{0}}, m_{S_{i}^{0}}$ in detail we start once again with the small to moderate $\boldsymbol{\lambda}$ scenario, as of the last section, and will address the remaining two scenarios successively.

\subsection{Regions of the parameter space with light scalars, pseudoscalars and neu- tralinos}

(a) Small to moderate $\lambda$ : for this range of $\boldsymbol{\lambda}$ values, as already discussed in the previous section, the extra contribution to the lightest doublet-like Higgs mass is small (see eq. (4.2)) even for small $\tan \beta$. For example with $\tan \beta=2$, the contribution varies between $\sim 0.03 \%$

\footnotetext{
${ }^{18}$ Note that the minimum of $M_{2}$ is $\approx 100 \mathrm{GeV}$ from the LEP lighter chargino mass bound [172].
} 
to $3 \%$ over that of the MSSM contribution as $\boldsymbol{\lambda}$ changes from 0.01 to 0.1 , respectively. Hence, a large stop mass and (or) a large $A$-term are much needed $[209,210]$ to produce a sizable loop correction to reach the target of $125 \mathrm{GeV}$, similar to the MSSM.

It is also worthy to note that for further small $\boldsymbol{\lambda}$ values (i.e. $\lesssim 0.01$ ) or in the limit of a vanishingly small $\boldsymbol{\lambda}$, eq. (5.5) coincides with the well-known NMSSM formulas of the same type [185] (although in the NMSSM one has only one singlet) and is given as

$$
m_{S_{i}^{0}}^{2} \approx 4 \kappa^{2} \nu^{c 2}+\kappa A_{k} \nu^{c}, \quad m_{P_{i}^{0}}^{2} \approx-3 \kappa A_{k} \nu^{c}, \quad m_{\widetilde{\chi}_{i+3}^{0}} \approx 2 \kappa \nu^{c} .
$$

So for this region of $\boldsymbol{\lambda}$ values, the mass scales for these states are solely determined by the parameters $\kappa, A_{\kappa}$ (parameter $\nu^{c}$ is estimated from $\mu=\sqrt{3} \boldsymbol{\lambda} \nu^{c}$ relation) and compositionwise they are completely free from any doublet contamination. These simple formulas can be utilised to estimate the concerned set of parameters. Note that from eq. (5.8) one can obtain the following relations between the masses:

$$
m_{S_{i}^{0}}^{2} \approx m_{\widetilde{\chi}_{i+3}^{0}}^{2}-m_{P_{i}^{0}}^{2} / 3, \quad m_{P_{i}^{0}}^{2} \approx-3 m_{\widetilde{\chi}_{i+3}^{0}} A_{\kappa} / 2 .
$$

Thus, the simultaneous presence of non-tachyonic $S_{i}^{0}$ and $P_{i}^{0}$ implies that $A_{\kappa}$ and $m_{\widetilde{\chi}_{i+3}^{0}}=$ $2 \kappa \nu^{c}$ must have the opposite signs, $m_{P_{i}^{0}}<\sqrt{3}\left|m_{\widetilde{\chi}_{i+3}^{0}}\right|$ and as a consequence $m_{S_{i}^{0}}<\left|m_{\widetilde{\chi}_{i+3}^{0}}\right|$. Hence, the light scalar/pseudoscalar states are assured when the light neutralinos are present. On the other hand, using the expression for $m_{P_{i}^{0}}^{2}$ in eq. (5.9) with the condition $m_{P_{i}^{0}}<\sqrt{3}\left|m_{\widetilde{\chi}_{i+3}^{0}}\right|$, one obtains that $\left|A_{\kappa}\right|<2\left|m_{\widetilde{\chi}_{i+3}^{0}}\right|$. Hence, for the light $\widetilde{\chi}_{i+3}^{0}$ (i.e., $\left.2\left|m_{\widetilde{\chi}_{i+3}^{0}}\right| \lesssim m_{S_{4}^{0}}\right)$, one can use this relation to estimate $\left|A_{\kappa}\right| \lesssim 125 \mathrm{GeV}$. If one demands $P_{i}^{0}$ states comparable/lighter than $\widetilde{\chi}_{i+3}^{0}$ states, then one gets $\left|A_{\kappa}\right| \lesssim 2\left|m_{\widetilde{\chi}_{i+3}^{0}}\right| / 3$. In this case $2\left|m_{\widetilde{\chi}_{i+3}^{0}}\right| \lesssim 125 \mathrm{GeV}$ predicts $\left|A_{\kappa}\right| \lesssim 42 \mathrm{GeV}$. It is thus apparent that the existence of scalar and/or pseudoscalar states lighter than $\widetilde{\chi}_{i+3}^{0}$ states requires small $A_{\kappa}$ values. The requirement is more stringent for lighter $P_{i}^{0}$ states.

Now before we start analysing the behaviours of $m_{\widetilde{\chi}_{i+3}^{0}}, m_{P_{i}^{0}}^{2}$ and $m_{P_{i}^{0}}^{2}$ in the light of eqs. ((5.5)-(5.8)), we want to emphasise that for the simplicity of the analysis: (1) we estimate the scale of $\nu^{c}$ using $\frac{\mu}{\sqrt{3} \lambda}$ relation with the minimum of $\mu \gtrsim 100 \mathrm{GeV}$, (2) we assume $A_{\lambda} \gg \kappa \nu^{c}$. The last assumption emerges from the fact that we need singlinos lighter than $m_{S_{4}^{0}}$ in order to affect the SM-like Higgs phenomenology through on-shell $S_{4}^{0} \rightarrow \widetilde{\chi}_{i+3}^{0} \widetilde{\chi}_{j+3}^{0}$ decay modes. The presence of the latter decay modes with $m_{\widetilde{\chi}_{i+3}^{0}}=2 \kappa \nu^{c}$ implies $\kappa \nu^{c} \lesssim 31.5 \mathrm{GeV}$. Hence together with $A_{\lambda}, \nu^{c}$ in the ballpark of a $\mathrm{TeV}$ (as preferred by the scale of soft-SUSY breaking masses), $A_{\lambda} \gg \kappa \nu^{c}$ is well justified. We work with $v=174 \mathrm{GeV}$.

1. We start with the neutralinos, where the expressions for $m_{\widetilde{\chi}_{U_{1,2}}^{0}}$ are free from $\boldsymbol{\lambda}$ parameter. They are also free from any doublet contamination. The mass scale for these neutralinos are determined by the parameters $\kappa$ and $\nu^{c}$. However, through the latter, $\lambda$-parameter dependency from $\mu=\sqrt{3} \lambda \nu^{c}$ relation implicitly enters in the evaluation of $m_{\widetilde{\chi}_{U_{1,2}}^{0}}$. One can, however, fixed the scale of $\nu^{c}$ to evade this implicit $\lambda$-dependence. The behaviour of their mass scale remains the same also when $\kappa_{i} \neq \kappa_{j}$ 
with $\kappa_{i}-\kappa_{j} \rightarrow 0$, although for this region of the parameter space they adhere a small to negligible doublet admixing. One should note that the relative position of $\widetilde{\chi}_{U_{1,2}}^{0}$ with respect to that of the $\widetilde{\chi}_{M}^{0}$ in the mass spectrum depends on the relative signs of the various parameters. For example, from eq. (5.7) with $\operatorname{sign}\left(\kappa \nu^{c}\right)=\operatorname{sign}\left(M_{1,2}\right)$, one gets $\left|m_{\widetilde{\chi}_{M}^{0}}\right| \lesssim\left|m_{\widetilde{\chi}_{U_{1,2}}^{0}}\right|$.

For the $\widetilde{\chi}_{M}^{0}$, from eq. (5.5) it is clear that the extra contribution goes from $\approx 50 \boldsymbol{\lambda}^{2} \times$ $\frac{(2.5-0.013 \mathcal{M})}{(1-0.008 \mathcal{M})}$ to $\approx 50 \boldsymbol{\lambda}^{2} \times \frac{(10.1-0.013 \mathcal{M})}{(1-0.033 \mathcal{M})}$ as $\tan \beta$ varies from 2 to 10 , taking the minimum of $\mu=100 \mathrm{GeV}$. Hence, with $\mathcal{M} \sim \mathcal{O}(100 \mathrm{GeV})$ and $\tan \beta=2$ one gets a contribution like $\approx 300 \lambda^{2} \mathrm{GeV}$ which yields a correction of around $3 \mathrm{GeV}$ with $\boldsymbol{\lambda}=0.1$. This contribution diminishes further with a larger values of $\mu, \tan \beta$ or $M_{1,2}$, i.e. larger $\mathcal{M}$. For example, reanalysis of the last step taking $\tan \beta=2$ and $\mathcal{M}=1 \mathrm{TeV}$ with everything else fixed gives a correction of $\approx 0.75 \mathrm{GeV}$. This correction reduces to $\approx$ $0.05 \mathrm{GeV}$ for $\tan \beta=10$. In other words, unless $2\left|\kappa \nu^{c}\right| \lesssim 10 \mathrm{GeV}$, for a novel region of the parameter space, the mass correction and the amount of doublet admixing remain negligible for $\widetilde{\chi}_{M}^{0}$. Thus, for most of the time $\left|m_{\widetilde{\chi}_{M}^{0}}\right| \approx\left|m_{\widetilde{\chi}_{U_{1,2}}^{0}}\right|$.

2. Turning towards $P_{i}^{0}$, the presence of multiple terms in the expressions of $m_{P_{U_{1,2}}^{0}}^{2}$ with the same coefficient $\boldsymbol{\lambda}^{2} v^{2}$ provides an option to remove the $\boldsymbol{\lambda}$ dependence from the mass terms for some specific set of the parameter choice. For small to moderate $\tan \beta$, the $\lambda$-dependent terms are given by $\delta m_{P_{U_{1,2}}^{0}}^{2}=\left[\left(\frac{A_{\lambda}}{\mu}+\frac{4 \kappa}{\sqrt{3} \lambda}\right) f(T)-1\right] \lambda^{2} v^{2}$. Now as we mentioned before, with $A_{\lambda}, \nu^{c} \sim \mathcal{O}(1 \mathrm{TeV}), \mu \sim \mathcal{O}(100 \mathrm{GeV})$ one gets $\frac{A_{\lambda}}{\mu} \approx 10$. At the same time, the light $\widetilde{\chi}_{U_{1,2}}^{0}$ in the upper limit (i.e., $2 m_{\widetilde{\chi}_{U_{1,2}}^{0}} \approx m_{S_{4}^{0}}$ ) together with $\nu^{c} \sim 1 \mathrm{TeV}$ predict $0.7 \leq \frac{4 \kappa}{\sqrt{3} \lambda} \lesssim 7$ for $0.1 \geq \lambda \gtrsim 0.01$. It is thus apparent that one needs at least $\kappa \lesssim 10^{-2}$ to use $\frac{A_{\lambda}}{\mu}+\frac{4 \kappa}{\sqrt{3} \lambda} \sim \frac{A_{\lambda}}{\mu}$. In this limit one effectively gets $\delta m_{P_{U_{1,2}}^{0}}^{2} \approx[10 f(T)-1] \lambda^{2} v^{2}$ assuming the relevant signs for the different parameters. The magnitude of this contribution is at most $\sim 0.1 \lambda^{2} v^{2}$ for $9 \leq \tan \beta \leq 11$ and vanishes ${ }^{19}$ around $\tan \beta \sim 9.9$. So in this corner of the parameter space the light $P_{U_{1,2}}^{0}$ are guaranteed with the proper choice of $\kappa, \nu^{c}$ and $A_{\kappa}$. Outside of this region, the lightness of $P_{U_{1,2}}^{0}$ are possible at the cost of a mutual cancellation between the different components in the expressions of $m_{P_{U_{1,2}}^{0}}^{2}$ (see eq. (5.5)). Note that with $\mu \gtrsim 100 \mathrm{GeV}, \frac{A_{\lambda}}{\mu} \sim 10 \Rightarrow A_{\lambda} \approx \nu^{c}$ when $\boldsymbol{\lambda} \sim \mathcal{O}(0.1)$ while $10 A_{\lambda} \approx \nu^{c}$ for $\lambda \sim \mathcal{O}(0.01)$.

With $A_{\lambda} \gg \kappa \nu^{c}$, the extra piece of contribution to $m_{P_{M}^{0}}^{2}$ as shown in eq. (5.5) goes as $\delta m_{P_{M}^{0}}^{2} \approx 3 \sqrt{3} f(T) \boldsymbol{\lambda} \kappa v^{2} \approx 1.58 \times 10^{5} f(T) \boldsymbol{\lambda} \kappa \mathrm{GeV}^{2}$. Now the scale for $\kappa$ can be estimated with $2\left|\kappa \nu^{c}\right| \lesssim m_{S_{4}^{0}} / 2$ and $\nu^{c} \approx \mathcal{O}(1 \mathrm{TeV})$, as $\lesssim \mathcal{O}\left(10^{-2}\right)$. On the other hand, $f(T)$ changes from 0.4 to $\sim 0.1$ as $\tan \beta$ varies from 2 to 10 , respectively. Thus, for $\tan \beta=2, \delta m_{P_{M}^{0}}^{2}$ goes as $\sim 632 \lambda \mathrm{GeV}^{2}$ which is about $63 \mathrm{GeV}^{2}$ for $\boldsymbol{\lambda}=0.1$.

\footnotetext{
${ }^{19}$ Since $\frac{A_{\lambda}}{\mu} f(T)-1=0$ is a quadratic equation in $\tan \beta$, one should expect another solution for $\tan \beta$, however, we do not consider any such solution when $\tan \beta<1$.
} 
This indicates that $\boldsymbol{\lambda}$-dependent contribution and hence the doublet admixing is nonnegligible for $P_{M}^{0}$. The lightness is, however, still possible using a possible cancellation between the two different terms in the expression of $m_{P_{M}^{0}}^{2}$ (see eq. (5.5)). One can of course consider $\tan \beta \gtrsim 10$ and/or a smaller $\kappa$ value to reduce $\delta m_{P_{M}^{0}}^{2}$ further.

3. Concerning the scalars, it is clear from eqs. (5.5) and (5.7) that it is in general rather hard to estimate the correction in $S_{M}^{0}$ from $\boldsymbol{\lambda}$ dependent terms, in the limit of small to moderate $\tan \beta$ (see eq. (5.5)). In general, one naively expects a non-negligible doublet impurity in $S_{M}^{0}$ for this range of $\boldsymbol{\lambda}$ values while the lightness of $S_{M}^{0}$, in an experimentally viable manner, is still possible with a fine cancellation among various components in the expression of $m_{S_{M}^{0}}^{2}$ (see eq. (5.5)).

For $S_{U_{1,2}}^{0}$, as shown in eq. (5.5), $\lambda$-dependent contributions are given by $\delta m_{S_{U_{1,2}}^{0}}^{2}=$ $\left[\frac{A_{\lambda}}{\mu} f(T)-1\right] \lambda^{2} v^{2}$, which are similar to the $\delta m_{P_{U_{1,2}}^{0}}^{2}$ assuming $\frac{A_{\lambda}}{\mu} \gg \frac{4 k}{\sqrt{3} \lambda}$. Hence, the analysis remains similar. We note in passing that another tool to reduce the contribution from the first term of $\delta m_{S_{U_{1,2}}^{0}}^{2}$ is to consider $A_{\lambda} \ll \mu$ while keeping $A_{\lambda} \gg \kappa \nu^{c}$ at the limit of a very small $\kappa$. In this case, for both of $S_{U_{1,2}}^{0}$ and $P_{U_{1,2}}^{0}$, the $\boldsymbol{\lambda}$-dependent term is given by $\boldsymbol{\lambda}^{2} v^{2}$ since $4 \kappa$ is also $\ll \sqrt{3} \boldsymbol{\lambda}$ for this region of the parameter space.

Combining all the facts, the lightness for $\widetilde{\chi}_{i+3}^{0}, P_{i}^{0}$ and $S_{U}^{0}$ states are rather assured in this region of $\boldsymbol{\lambda}$ values with a negligible to small tuning of the other parameters. Concerning the $S_{M}^{0}$, especially for $\boldsymbol{\lambda} \sim 0.1$, a low mass is rather hard to accommodate without a fine cancellation between the different contributors. A similar conclusion also holds true for the amount of doublet impurity in $S_{M}^{0}$. The amount of the doublet admixing in the $P_{M}^{0}$ and $\widetilde{\chi}_{M}^{0}$, on the other hand, are rather easily controlled with a proper but not very fine tuned choice of the other model parameters.

(b) Moderate to large $\lambda$ : moving towards moderate to large $\boldsymbol{\lambda}$ region, as mentioned in section 4 , the additional contribution to the tree-level lightest doublet-like scalar mass over the same from the MSSM can vary from $12 \%$ to $\sim 100 \%$ when $\boldsymbol{\lambda}$ goes from 0.2 to 0.7 with $\tan \beta=2$. With increasing $\tan \beta$, once again this extra contribution goes down, for example $\sim 4 \%$ for $\boldsymbol{\lambda}=0.7$ with $\tan \beta=10$. Necessity of a large stop mass and/or a large $A$-term are somewhat ameliorated for this scenario in the region of $\tan \beta \lesssim 10$. Further, in this corner of the parameter space an enhanced branching ratio is possible for $S_{4}^{0} \rightarrow \gamma \gamma$ compared to the SM, especially as $\boldsymbol{\lambda}$ tends to 0.7 with a suitable choice of the other parameters. This enhancement is supported by both the ATLAS [19] and CMS collaborations [17] to date.

In this region of the parameter space, the lightness of $S_{U_{1,2}}^{0}$ and $P_{U_{1,2}}^{0}$ are not assured without a moderate tuning of the relevant parameters (e.g., $\boldsymbol{\lambda},|\kappa|,\left|A_{\kappa}\right|$ etc.). Their purities, however, remain unaffected by the virtue of the construction.

Before beginning the discussion of $\widetilde{\chi}_{i+3}^{0}, P_{i}^{0}$ and $S_{i}^{0}$ masses, note that for this range of $\boldsymbol{\lambda}$ values, the estimation of $\nu^{c}$ from $\mu / \sqrt{3} \boldsymbol{\lambda}$ relation with $\mu$ around $100 \mathrm{GeV}$ is somewhat inconsistent with the $\mathrm{TeV}$-scale soft masses. For example, with this assumption one 
would get $\nu^{c} \approx 80 \mathrm{GeV}$ for $\boldsymbol{\lambda}=0.7$ and so we fixed $\nu^{c}$ at $1 \mathrm{TeV}$ for this region of the parameter space.

1. Discussion for $m_{\widetilde{\chi}_{U_{1,2}}^{0}}$ remain the same as before while interpretation for the $m_{\widetilde{\chi}_{M}^{0}}$ is more involved than the one for the small to moderate $\boldsymbol{\lambda}$ region with $\nu^{c}$ fixed at $1 \mathrm{TeV}$.

(i) In the region of the parameter space with large $\mu$ or $\mathcal{M}$ (such that $\frac{\mathcal{M} \mu}{v^{2}} \gg \tan \beta$ ), the extra term, $\delta m_{\widetilde{\chi}_{M}^{0}}$ is written as $\frac{2 \boldsymbol{\lambda} v^{2}}{3 \sqrt{3} \nu^{c}} \times \frac{T}{\left(1+T^{2}\right)}$. This term changes from $\sim 1 \mathrm{GeV}$ to $\sim 3 \mathrm{GeV}$ when $\boldsymbol{\lambda}$ changes from 0.2 to 0.7 for $\nu^{c}=1 \mathrm{TeV}$ and $\tan \beta=2$. Thus, unless $2 \kappa \nu^{c}$ is very small $(\lesssim 10 \mathrm{GeV})$, effect from this new term in the lightness of $m_{\widetilde{\chi}_{M}^{0}}$ is small to negligible and its effect in spoiling the singlet purity of $\widetilde{\chi}_{M}^{0}$ is also moderate to suppressed. A Larger value of $\tan \beta$ is another way to reduce this extra contribution.

(ii) Now we investigate another corner of the parameter space with $\mathcal{M} \approx \mu \approx v$ where the new contribution, $\delta m_{\widetilde{\chi}_{M}^{0}}$ goes as $\approx-\frac{\boldsymbol{\lambda} v^{2}}{6 \sqrt{3} \nu^{c}} \frac{\left(1+T^{2}-4 T\right)}{\left(1+T^{2}-T\right)}$, or numerically $\sim 3 \boldsymbol{\lambda}$ with $\tan \beta=2$ and $\nu^{c}=1 \mathrm{TeV}$. Thus, as $\boldsymbol{\lambda}$ changes from 0.2 to 0.7 , this varies from $\sim 0.6$ to $\sim 2 \mathrm{GeV}$ and decreases further for larger $\tan \beta$ values. Consequently its contribution to the $\widetilde{\chi}_{M}^{0}$ mass term as well to the composition from the doublet-like states remains negligible unless $\left|2 \kappa \nu^{c}\right| \lesssim 10 \mathrm{GeV}$. Note that the sign of this contribution changes for $\tan \beta \geq 4$ when it appears as a negative one.

2. Concerning the $P_{i}^{0}$, the situation remains flexible in order to secure light the $P_{i}^{0}$ states or to minimise the doublet composition in $P_{M}^{0}$.

In this range of $\boldsymbol{\lambda}$ values $4 \kappa / \sqrt{3} \boldsymbol{\lambda}$ decreases further compared to the last region (i.e., about $0.1 \leq \frac{4 \kappa}{\sqrt{3} \lambda} \lesssim 0.4$ for $0.7 \geq \lambda \gtrsim 0.2$ ) and hence, just like the small to moderate $\boldsymbol{\lambda}$ scenario, the $\boldsymbol{\lambda}$-dependent contribution are given by $\delta m_{P_{U_{1,2}}^{0}}^{2} \approx\left[\frac{A_{\lambda}}{\mu} f(T)-1\right] \boldsymbol{\lambda}^{2} v^{2}$. However, now with $A_{\lambda}, \nu^{c} \approx \mathcal{O}(1 \mathrm{TeV})$, one gets $350 \mathrm{GeV} \lesssim \mu \lesssim 1200 \mathrm{GeV}$ as $\boldsymbol{\lambda}$ moves from 0.2 to 0.7 . With our choice of $A_{\lambda}, \nu^{c}$, the quantity $A_{\lambda} / \mu$ varies between $\sim 0.8$ to 2.8 and hence depending on the value of $\tan \beta$ this $\boldsymbol{\lambda}$-dependent contribution may appear negligible. For example, with $\boldsymbol{\lambda}=0.2$, magnitude of this extra contribution is about $0.1 \times \boldsymbol{\lambda}^{2} v^{2}$ for $2 \lesssim \tan \beta \lesssim 2.9$ and vanishes around $\tan \beta \approx 2.38$. With $\boldsymbol{\lambda}=0.7$, keeping $\nu^{c}$ fixed at $1 \mathrm{TeV}$, a similar phenomenon remains missing for any real values of $\tan \beta$. Nevertheless, depending on the relative signs of the different terms with the proper choice of parameters, e.g. $\kappa, \nu^{c}$ and $A_{\kappa}$, the light $P_{U_{1,2}}^{0}$ are well affordable in this corner of the parameter space, at the cost of a partial cancellation between the different components.

Moving towards $P_{M}^{0}$, with $A_{\lambda} / \nu^{c} \approx 1$, the $\lambda$ dependent correction, $\delta m_{P_{M}^{0}}^{2}$ is $\approx$ $3 \sqrt{3} f(T) \times \boldsymbol{\lambda} \kappa v^{2} \approx 6.3 \times 10^{4} \boldsymbol{\lambda} \kappa \mathrm{GeV}^{2}$ with $\tan \beta=2$. Larger $\tan \beta$ values of course provide an additional tool to reduce this contribution. The quantity $\delta m_{P_{M}^{0}}^{2}$ varies from $\sim 1.3 \times 10^{4} \kappa \mathrm{GeV}^{2}$ to $\sim 4.4 \times 10^{4} \kappa \mathrm{GeV}^{2}$ as $\boldsymbol{\lambda}$ goes from 0.2 to 0.7 . It is clear now that if we stick to $\kappa \sim \mathcal{O}\left(10^{-2}\right)$ (as guided by $\left|2 \kappa \nu^{c}\right| \lesssim m_{S_{4}^{0}} / 2$ ), these corrections 
are significant, e.g. about $130 \mathrm{GeV}^{2}$ for $\boldsymbol{\lambda}=0.2$. So we need to move to the region of $\kappa \lesssim 10^{-3}$ to reduce this extra contribution so that the lightness and the singlet purity for $P_{M}^{0}$ remain assured for this region of the parameter space with a proper choice of the other relevant parameters (e.g., $A_{\kappa}$ ). The choice of $\kappa \sim \mathcal{O}\left(10^{-3}\right.$ ) also makes the assumption $\frac{A_{\lambda}}{\mu} f(T)+\frac{4 \kappa}{\sqrt{3} \lambda} \approx \frac{A_{\lambda}}{\mu}$ more reliable. One would, however, need a higher $\nu^{c}$ value to get $2 \kappa \nu^{c} \gtrsim 10 \mathrm{GeV}$.

We note in passing that it is still possible to accommodate a light $P_{M}^{0}$ with $\kappa \sim 10^{-2}$ at the cost of a cancellation between the different parts in the expression of $m_{P_{M}^{0}}^{2}$.

3. Once again like the earlier $\boldsymbol{\lambda}$ scenario, an interpretation about the singlet purity and the lightness for $S_{M}^{0}$ is highly complicated. The situation gets worse as one moves to higher $\boldsymbol{\lambda}$ values. The only possibility to assure a light $S_{M}^{0}$ for this corner of the parameter space appears through a cancellation among the various terms in the expression of $m_{S_{M}^{0}}^{2}$, at the cost of a large fine tuning of the different relevant parameters.

Moving towards $S_{U_{1,2}}^{0}$, the $\boldsymbol{\lambda}$-dependent contributions are the same as that of the pair of $P_{U_{1,2}}^{0}$ with the assumption $\frac{A_{\mu}}{\mu} \gg \frac{4 \kappa}{\sqrt{3} \lambda}$. Hence, the discussion remains the same as that of the $P_{U_{1,2}}^{0}$.

Summarising the discussion, we conclude that the light singlet-like $\widetilde{\chi}_{i+3}^{0}$ states are well feasible in this range of $\boldsymbol{\lambda}$ values without a large parameter tuning or a strange cancellation. A light singlet-like $P_{M}^{0}$ appears with a bit of parameter tuning, especially for $|\kappa|$, however, more easily compared to the light $P_{U_{1,2}}^{0}$ or $S_{U_{1,2}}^{0}$. The presence of the light $P_{U_{1,2}}^{0}$ and $S_{U_{1,2}}^{0}$ even for $\boldsymbol{\lambda}=0.2$, requires certain degree of parameter tuning which grows with increasing $\lambda$. A singlet-like $S_{M}^{0}$ is rather hard in this region without a high amount of parameter tuning. A similar argument holds true for the lightness of $m_{S_{M}^{0}}$. Note that in this region of the $\boldsymbol{\lambda}$ values or higher, the existence of a light $S_{M}^{0}$ for $\boldsymbol{\lambda}>0.1$ and large $\tan \beta$ excludes the possibility of having the light $P_{i}^{0}, \widetilde{\chi}_{M}^{0}$ in the mass spectrum. In a similar fashion, for the same corner of the parameter space, the presence of the light $P_{i}^{0}, \widetilde{\chi}_{i+3}^{0}$ states discards the existence of a light $S_{M}^{0}$.

(c) Dominant $\lambda$ : in this region of the parameter space, as already addressed in section 4, one can really accommodate a $125 \mathrm{GeV}$ Higgs boson even with only tree-level contribution, at the cost of relaxing perturbativity of $\boldsymbol{\lambda}$ up to the GUT scale. Hence, a small $A$-term and(or) small stop masses are well affordable in this scenario. Assuming perturbativity up to $10 \mathrm{TeV}, \boldsymbol{\lambda}$ as large as 1.7 is possible, as can be interpreted from figure 2 . With this scenario an enhancement over the MSSM tree-level Higgs mass as large as $\approx 7 \%$ is possible with $\tan \beta=10$ and $\boldsymbol{\lambda}=1$. At lower $\tan \beta$, say 5 , this contribution gives $\approx 28 \%$ enhancement over the MSSM limit. A larger contribution for a higher $\boldsymbol{\lambda}$ values is possible with the larger $\tan \beta$ values as shown in figure 2 , although constraints from flavour observables can restrict this scenario.

Similar to the moderate to large $\boldsymbol{\lambda}$ region, we do not determine the scale of $\nu^{c}$ using $\mu=\sqrt{3} \lambda \nu^{c}$ relation for this corner of the parameter space as then $\left.\mu\right|_{\min } \sim 100 \mathrm{GeV}$ would 
predict $\left|\nu^{c}\right| \lesssim 60 \mathrm{GeV}$ for $\boldsymbol{\lambda} \gtrsim 1$. Note that, however, here keeping $\left|\nu^{c}\right|$ fixed at $1 \mathrm{TeV}$ would give $\mu \approx 2 \mathrm{TeV}$ for $\boldsymbol{\lambda}=1.2$. Hence, here we keep $\mu$ fixed at $1 \mathrm{TeV}$.

1. Concentrating on the neutralinos, behaviour of the two $m_{\widetilde{\chi}_{U_{1,2}}^{0}}$ remain the same as of the last two $\boldsymbol{\lambda}$ regions. For $m_{\widetilde{\chi}_{M}^{0}}$ the discussion is also similar to the last $\boldsymbol{\lambda}$ region, although the amount of $\boldsymbol{\lambda}$-dependent correction increases in the magnitude due to the larger $\boldsymbol{\lambda}$ values.

(i) In the corner of the parameter space with the large $\mu$ or $\mathcal{M}$ (such that $\frac{\mathcal{M} \mu}{v^{2}} \gg$ $\tan \beta$ ), this extra term, i.e. $\frac{2 \boldsymbol{\lambda}^{2} v^{2}}{3 \mu} \times \frac{T}{\left(1+T^{2}\right)}$ for $\tan \beta=2,\left|\nu^{c}\right|=1 \mathrm{TeV}$ and $\boldsymbol{\lambda}=1$ is estimated as $\sim 8 \mathrm{GeV}$. Hence, unless $\left|2 \kappa \nu^{c}\right| \lesssim 10 \mathrm{GeV}$, effect from this new term in the lightness of $m_{\widetilde{\chi}_{M}^{0}}$ and also in determining the amount of the doublet impurity in $\tilde{\chi}_{M}^{0}$ remains moderate to small. Larger values for $\tan \beta$ is another tool to reduce the doublet contamination in $\widetilde{\chi}_{M}^{0}$ apart from ensuring its lightness with the suitable $|\kappa|$ and $\left|\nu^{c}\right|$ values.

(ii) In the region of the parameter space with $\mathcal{M} \approx \mu \approx v$ the new contribution, $-\frac{\boldsymbol{\lambda}^{2} v^{2}}{6 \mu} \times \frac{\left(1+T^{2}-4 T\right)}{\left(1+T^{2}-T\right)}$ goes as $\sim 5 \mathrm{GeV}$ with $\boldsymbol{\lambda}=1, \tan \beta=2$ and $\nu^{c}=1 \mathrm{TeV}$. This is again a small contribution unless $\left|2 \kappa \nu^{c}\right| \lesssim 10 \mathrm{GeV}$. So the effect of this term in the lightness of $\widetilde{\chi}_{M}^{0}$ and in the doublet composition of $\widetilde{\chi}_{M}^{0}$ remains moderate to small, especially for larger $\tan \beta$ values.

2. The discussion with $P_{i}^{0}$ states are very similar to that of the last $\boldsymbol{\lambda}$ region. With $|\kappa| \sim \mathcal{O}\left(10^{-3}\right)$ and $\boldsymbol{\lambda} \gtrsim 1, \frac{4 \kappa}{\sqrt{3} \boldsymbol{\lambda}} \lesssim 0.0023$. Thus, with $A_{\lambda}, \mu \approx \mathcal{O}(1 \mathrm{TeV}), \frac{A_{\lambda}}{\mu} \gg \frac{4 \kappa}{\sqrt{3} \boldsymbol{\lambda}}$ is well justified and we end up with $\delta m_{P_{U_{1,2}}^{0}}^{2} \approx[f(T)-1] \lambda^{2} v^{2}=3[f(T)-1] \times 10^{4} \mathrm{GeV}^{2}$ for $\boldsymbol{\lambda}=1$. This contribution does not vanish for any real values of $\tan \beta$, however, $\lesssim \lambda^{2} v^{2}$ for small $\tan \beta$ values. So the lightness of $P_{U_{1,2}}^{0}$ are difficult for this corner of the parameter space without a large cancellation between the different components in the expressions of $m_{P_{U_{1,2}}^{0}}^{2}$ (see eq. (5.5)) with a proper choice of the other relevant parameters.

Regarding $P_{M}^{0}$, with $A_{\lambda} /\left(A_{\lambda}+\kappa \nu^{c}\right) \approx 1$, the $\lambda$-dependent contribution in $\delta m_{P_{M}^{0}}^{2}$ is given by $\approx 3 \sqrt{3} f(T) \boldsymbol{\lambda} \kappa v^{2} \approx 1.57 \times 10^{2} f(T) \mathrm{GeV}^{2}$ with $\boldsymbol{\lambda}=1$ and $\kappa \sim 10^{-3}$. This is clearly a non-negligible correction unless one moves to large $\tan \beta$ values $(\gtrsim 10)$. Hence, the lightness and the singlet purity are not generic to $P_{M}^{0}$ for this region of $\boldsymbol{\lambda}$ values. However, with a suitable sign choice of the relevant parameters it remains possible to yield a light $P_{M}^{0}$ using a moderate to large cancellation between the different components in the expression of $m_{P_{M}^{0}}^{2}$.

3. Concerning $S_{i}^{0}$, it is in general hard to accommodate a light $S_{M}^{0}$ for this region of the parameter space, especially for small to moderate $\tan \beta$, without a severe cancellation among the various components in the expression of $m_{S_{M}^{0}}^{2}$ (see eq. (5.5)). This large cancellation also indicates a very high doublet contamination in $S_{M}^{0}$. 
Regarding the two $S_{U_{1,2}}^{0}$ the analysis is the same as that of the two $P_{U_{1,2}}^{0}$ with the valid assumption $\frac{A_{\lambda}}{\mu} \gg \frac{4 \kappa}{\sqrt{3} \lambda}$. The lightness of $m_{S_{U_{1,2}}^{0}}^{2}$ for this region of $\boldsymbol{\lambda}$ values, appears mainly with a large possible cancellation between the different terms in the expressions of $m_{S_{1,2}^{0}}^{2}$.

In summary, the simultaneous presence of light $\widetilde{\chi}_{i+3}^{0}, S_{i}^{0}$ and $P_{i}^{0}$ states are hardly possible in the dominant $\boldsymbol{\lambda}$ region. Concerning the lightness of all the states and singlet purity of the mixed states, the neutralinos appear as the most favoured ones in terms of the amount of fine tuning of the parameters. The pseudoscalars $P_{i}^{0}$ as well as $S_{U_{1,2}}^{0}$ are second on the list with a moderate to large fine tuning, for small to moderate $\tan \beta$ values. A pure $S_{M}^{0}$ is hardly possible for this range of $\boldsymbol{\lambda}$ values although the lightness can be achieved with a large to severe tuning of the relevant parameters.

In a nutshell, so far we have given a complete overview of the relevant parameters, not only to accommodate a $125 \mathrm{GeV}$ SM Higgs-like scalar boson, but at the same time to investigate the possibility of having the light singlet-like scalars, pseudoscalars and neutralinos in the mass spectrum. Thus, it remains to address the only remaining part of our analysis, namely the effects of the aforesaid light states in the decay phenomenology of the SM Higgs-like $S_{4}^{0}$. We aim to address these issues in the next section, once again giving special emphasis on the three different $\boldsymbol{\lambda}$ regions.

\section{New decays of the SM-like Higgs in the $\mu \nu \mathrm{SSM}$}

In this section we present analytical estimates of the decays of the SM Higgs-like $S_{4}^{0}$ into a pair of $S_{i}^{0}, P_{i}^{0}$ and $\widetilde{\chi}_{i+3}^{0}$ states. Note that we consider only new two-body decays of $S_{4}^{0}$ and thus, more complex or longer decay cascades like the ones addressed in ref. [49] will be skipped. It will be useful to compute first the complete expressions of the decay widths for these processes as:

$$
\begin{aligned}
\Gamma_{S_{4}^{0} \rightarrow S_{i}^{0} S_{j}^{0}}= & \left|\widetilde{g} O_{4 i j}^{S S S}\right|^{2} \times \frac{\mathcal{F}\left(m_{S_{4}^{0}}^{2}, m_{S_{i}^{0}}^{2}, m_{S_{j}^{0}}^{2}\right)}{16 \pi m_{S_{4}^{0}}\left(1+\delta_{i j}\right)}, \\
\Gamma_{S_{4}^{0} \rightarrow P_{i}^{0} P_{j}^{0}}= & \left|\widetilde{g} O_{4 i j}^{S P P}\right|^{2} \times \frac{\mathcal{F}\left(m_{S_{4}^{0}}^{2}, m_{P_{i}^{0}}^{2}, m_{P_{j}^{0}}^{2}\right)}{16 \pi m_{S_{4}^{0}}\left(1+\delta_{i j}\right)}, \\
\Gamma_{S_{4}^{0} \rightarrow \widetilde{\chi}_{i+3}^{0} \widetilde{\chi}_{j+3}^{0}=} & {\left[\left(\frac{1}{2}\left|\widetilde{g} O_{L(i+3)(j+3) 4}^{n n h}\right|^{2}+\frac{1}{2}\left|\widetilde{g} O_{R(i+3)(j+3) 4}^{n n h}\right|^{2}\right)\left(m_{S_{4}^{0}}^{2}-m_{\widetilde{\chi}_{i+3}^{0}}^{2}-m_{\widetilde{\chi}_{j+3}^{0}}^{2}\right)\right.} \\
& \left.-2 \Re\left(|\widetilde{g}|^{2} O_{L(i+3)(j+3) 4}^{n n h} O_{R(i+3)(j+3) 4}^{n n h^{*}}\right) m_{\widetilde{\chi}_{i+3}^{0}} m_{\widetilde{\chi}_{j+3}^{0}}\right] \\
& \times \frac{\mathcal{F}\left(m_{S_{4}^{0}}^{2}, m_{\widetilde{\chi}_{i+3}^{0}}^{2}, m_{\widetilde{\chi}_{j+3}^{0}}^{2}\right)}{16 \pi m_{S_{4}^{0}}\left(1+\delta_{i j}\right)} .
\end{aligned}
$$

Here $m_{S_{4}^{0}}^{2} \mathcal{F}\left(m_{S_{4}^{0}}^{2}, m_{X_{i}^{0}}^{2}, m_{X_{j}^{0}}^{2}\right)=\sqrt{\left(m_{S_{4}^{0}}^{2}-m_{X_{i}^{0}}^{2}-m_{X_{j}^{0}}^{2}\right)^{2}-4 m_{X_{i}^{0}}^{2} m_{X_{j}^{0}}^{2}}$ with $X_{i}^{0}=S_{i}^{0}, P_{i}^{0}$ and $\widetilde{\chi}_{i+3}^{0}$, the couplings $\widetilde{g} O_{m n p}^{S S S}$ and $\widetilde{g} O_{m n p}^{S P P}$ are complicated functions which are given in 

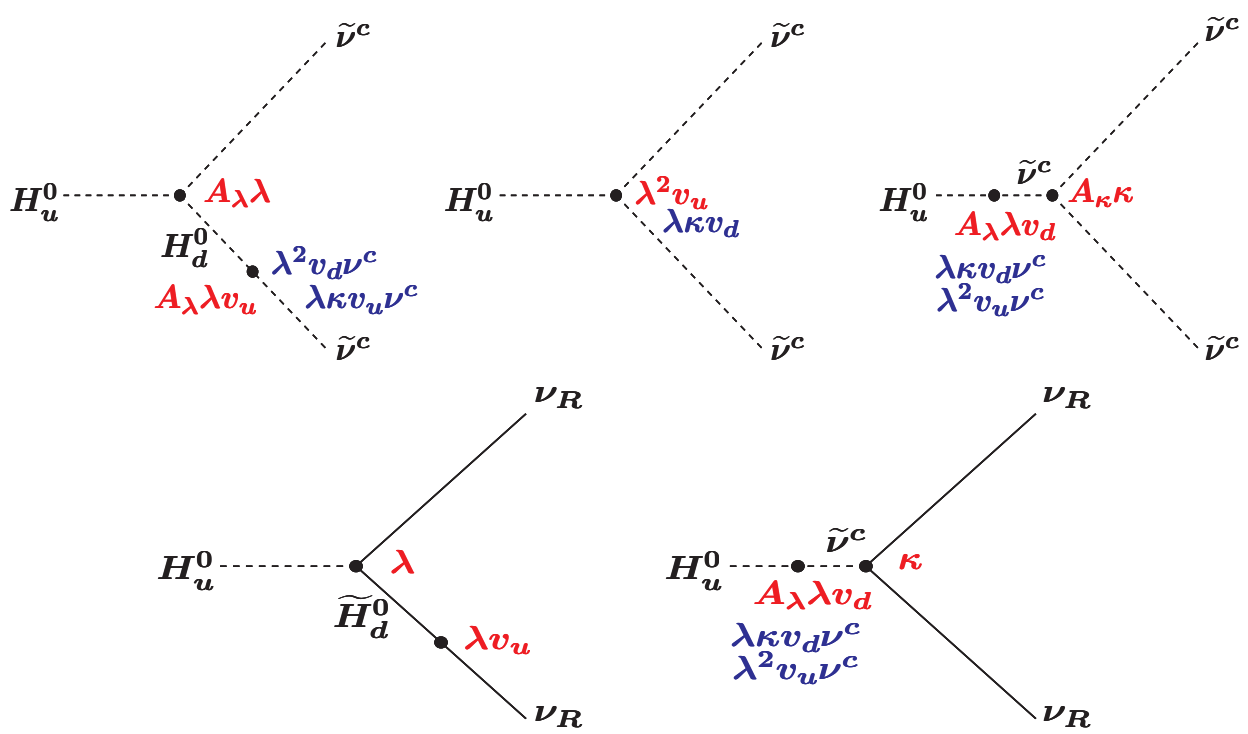

Figure 3. Diagrams showing SM-like $S_{4}^{0}$ decays into a pair of singlet-like CP-even scalars, CP-odd scalars and neutralinos in the flavour basis with the leading contributions. Symbol $\nu_{R}$ has been used to represent a right-handed neutrino. Red (blue) colour has been used to represent couplings of certain (alternate) kind. An extra factor that appears when a complex scalar field $\boldsymbol{\Phi}$ is decomposed as $\boldsymbol{\Phi}=v_{\Phi}+\frac{\Re \boldsymbol{\Phi}+i \Im \Phi}{\sqrt{2}}$ with $v_{\Phi}$ as the acquired $\mathrm{VEV}$, is not explicitly shown here. Diagrams with $Y_{\nu_{i j}}$ or $\nu_{i}$ in the couplings are not shown since they give rise to negligible contributions.

the appendix B of ref. [49], with a notation $h_{\delta} h_{\epsilon} h_{\eta} \equiv \widetilde{g} O_{\delta \epsilon \eta}^{S S S}$ and $h_{\delta} P_{\epsilon} P_{\eta} \equiv \widetilde{g} O_{\delta \epsilon \eta}^{S P P}$, and the couplings $\widetilde{g} O_{L i j k}^{n n h}$ and $\widetilde{g} O_{R i j k}^{n n h}$ are given in the appendix E of ref. [32]. Note that the kinematic factor $\mathcal{F}\left(m_{S_{4}^{0}}^{2}, m_{X_{i}^{0}}^{2}, m_{X_{j}^{0}}^{2}\right) \approx 1$ for $m_{S_{i}^{0}}, m_{P_{i}^{0}}, m_{\widetilde{\chi}_{i+3}^{0}} \ll m_{S_{4}^{0}}$. This holds roughly true for higher values of $m_{X_{i}^{0}}$ also, e.g. $\mathcal{F}\left(m_{S_{4}^{0}}^{2}, m_{X_{i}^{0}}^{2}, m_{X_{j}^{0}}^{2}\right) \sim 0.83$ for $m_{X_{i}^{0}}=35 \mathrm{GeV}$.

At this point we want to stress that since our goal is to describe a complete picture of the possible new two-body $S_{4}^{0}$ decay phenomenology with the $\mu \nu \mathrm{SSM}$, our analyses are confined up to the level of analytical estimates. Note that a full numerical analysis using eq. (6.1), as anticipated in a set of forthcoming publications [183], should satisfy a class of existing experimental observations [6, 8-12, 14, 17, 19-21, 106, 108, 128-130, 133, 147, 214-217].

Assuming $S_{i}^{0}, P_{i}^{0}, \widetilde{\chi}_{i+3}^{0}$ with a leading ${ }^{20}$ singlet composition and the SM-like $S_{4}^{0}$, we present diagrams giving leading contributions to $S_{4}^{0} \rightarrow S_{i}^{0} S_{j}^{0}, P_{i}^{0} P_{j}^{0}, \widetilde{\chi}_{i+3}^{0} \widetilde{\chi}_{j+3}^{0}$ processes in figure 3. We adopt the flavour basis for the convenience of analysis. We emphasise here that these simple analytical analyses are purely qualitative although agreed rather well with the full numerical results. However, when the amount of doublet impurity is high in $S_{i}^{0}, P_{i}^{0}, \widetilde{\chi}_{i+3}^{0}$ (e.g., for larger $\boldsymbol{\lambda}$ values), these estimations differ significantly.

Figure 3 in the mass or physical basis represents $S_{4}^{0} \rightarrow S_{i}^{0} S_{j}^{0}, P_{i}^{0} P_{j}^{0}$ and $\widetilde{\chi}_{i+3}^{0} \widetilde{\chi}_{j+3}^{0}$ processes. Following our discussion of section 5, especially for the chosen set of parameters (see

\footnotetext{
${ }^{20}$ In figure 3, we label a state as X-like when the composition of X in that state dominates ( $\gtrsim 90 \%$ ) over the others. For example, a SM-like $S_{4}^{0}$ requires leading $H_{u}^{0}$ composition although certain amount of $H_{d}^{0}$ component is essential so that it can couple to the down-type fermions, e.g. $b \bar{b}, \tau^{+} \tau^{-}$, etc.
} 
eq. (5.4)), it is clear that two of these $S_{i}^{0}, P_{i}^{0}, \widetilde{\chi}_{i+3}^{0}$ states are $S_{U}^{0}, P_{U}^{0}$ and $\widetilde{\chi}_{U}^{0}$, respectively, while the remaining $S_{i}^{0}, P_{i}^{0}, \widetilde{\chi}_{i+3}^{0}$ states represent $S_{M}^{0}, P_{M}^{0}, \widetilde{\chi}_{M}^{0}$. It is thus, important to emphasise here that all of these states do not couple to $S_{4}^{0}$ with identical strengths. To start with, it is convenient first to write down all the relevant terms used to draw figure 3. Following ref. [23] they are: ${ }^{21}$

$$
\begin{aligned}
\mathcal{L}= & {\left[-\left(A_{\lambda} \lambda\right)_{i} \widetilde{\nu}_{i}^{c} H_{u}^{0} H_{d}^{0}+\frac{1}{3}\left(A_{\kappa} \kappa\right)_{i j k} \widetilde{\nu}_{i}^{c} \widetilde{\nu}_{j}^{c} \widetilde{\nu}_{k}^{c}+\kappa_{i j k} \lambda_{j}^{*} H_{u}^{0^{*}} H_{d}^{0^{*}} \widetilde{\nu}_{i}^{c} \widetilde{\nu}_{k}^{c}+\text { H.c. }\right] } \\
& +\lambda_{i} \lambda_{j}^{*}\left(H_{u}^{0} H_{u}^{0^{*}}+H_{d}^{0} H_{d}^{0^{*}}\right) \widetilde{\nu}_{i}^{c} \widetilde{\nu}_{j}^{c^{*}}+\kappa_{i j k} \kappa_{l j m} \widetilde{\nu}_{i}^{c} \widetilde{\nu}_{l}^{c^{*}} \widetilde{\nu}_{k}^{c} \widetilde{\nu}_{m}^{c^{*}} \\
& +\frac{1}{2}\left[\lambda_{i} H_{u}^{0} \widetilde{H}_{d}^{0} \nu_{R_{i}}-2 \kappa_{i j k} \widetilde{\nu}_{i}^{c} \nu_{R_{j}} \nu_{R_{k}}+\text { H.c. }\right] .
\end{aligned}
$$

Assuming real parameters, eq. (6.2) in the light of eq. (5.4) can be rewritten as

$$
\begin{aligned}
\mathcal{L}= & {\left[-\frac{A_{\lambda} \boldsymbol{\lambda}}{\sqrt{3}} H_{u}^{0} H_{d}^{0} \sum_{i=1}^{3} \widetilde{\nu}_{i}^{c}+\frac{1}{3} A_{\kappa} \kappa \sum_{i=1}^{3} \widetilde{\nu}_{i}^{c^{3}}+\frac{\kappa \boldsymbol{\lambda}}{\sqrt{3}} H_{u}^{0^{*}} H_{d}^{0^{*}} \sum_{i=1}^{3} \widetilde{\nu}_{i}^{c^{2}}+\text { H.c. }\right] } \\
& +\frac{\lambda^{2}}{3}\left(H_{u}^{0} H_{u}^{0^{*}}+H_{d}^{0} H_{d}^{0^{*}}\right) \sum_{i=1}^{3} \widetilde{\nu}_{i}^{c} \sum_{j=1}^{3} \widetilde{\nu}_{j}^{c^{*}}+\kappa^{2} \sum_{i=1}^{3}\left|\widetilde{\nu}_{i}^{c}\right|^{4} \\
& +\frac{1}{2}\left[\frac{\lambda}{\sqrt{3}} H_{u}^{0} \widetilde{H}_{d}^{0} \sum_{i=1}^{3} \nu_{R_{i}}-2 \kappa \sum_{i=1}^{3} \widetilde{\nu}_{i}^{c} \nu_{R_{i}}^{2}+\text { H.c. }\right] .
\end{aligned}
$$

Following the footnote 17, it is possible to relate $\widetilde{\nu}_{i}^{c}$ and $\nu_{R_{i}}$ states with $S_{M}^{0}, S_{U_{1,2}}^{0}, P_{M}^{0}, P_{U_{1,2}}^{0}$ and $\widetilde{\chi}_{M}^{0}, \widetilde{\chi}_{U_{1,2}}^{0}$ states, respectively, as:

$$
\Re \widetilde{\nu}^{c}=\mathcal{U} S^{0^{\prime}}, \quad \Im \widetilde{\nu}^{c}=\mathcal{U} P^{0^{\prime}}, \quad \nu_{R}=\mathcal{U} \widetilde{\chi}^{0^{\prime}},
$$

where $\Re \widetilde{\nu}^{c}=\left(\Re \widetilde{\nu}_{1}^{c}, \Re \widetilde{\nu}_{2}^{c}, \Re \widetilde{\nu}_{3}^{c}\right), \Im \widetilde{\nu}^{c}=\left(\Im \widetilde{\nu}_{1}^{c}, \Im \widetilde{\nu}_{2}^{c}, \Im \widetilde{\nu}_{3}^{c}\right), \nu_{R}=\left(\nu_{R_{1}}, \nu_{R_{2}}, \nu_{R_{3}}\right), S^{0^{\prime}}=$ $\left(S_{M}^{0}, S_{U_{1}}^{0}, S_{U_{2}}^{0}\right), P^{0^{\prime}}=\left(P_{M}^{0}, P_{U_{1}}^{0}, P_{U_{2}}^{0}\right), \widetilde{\chi}^{0^{\prime}}=\left(\widetilde{\chi}_{M}^{0}, \widetilde{\chi}_{U_{1}}^{0}, \widetilde{\chi}_{U_{2}}^{0}\right)$ are all $3 \times 1$ matrices. The $3 \times 3$ matrix $\mathcal{U}$, following the footnote 17 , is given by

$$
\mathcal{U}=\left(\begin{array}{ccc}
\frac{1}{\sqrt{3}} & \frac{1}{\sqrt{2}} & \frac{1}{\sqrt{6}} \\
\frac{1}{\sqrt{3}} & 0 & -\sqrt{\frac{2}{3}} \\
\frac{1}{\sqrt{3}} & -\frac{1}{\sqrt{2}} & \frac{1}{\sqrt{6}}
\end{array}\right)
$$

Note that these transformations give $\sum \widetilde{\nu}_{i}^{c}=\sum \nu_{i}^{c}+\left(\Re \widetilde{\nu}_{i}^{c}+i \Im \widetilde{\nu}_{i}^{c}\right) / \sqrt{2}=3 \nu^{c}+\sqrt{3}\left(S_{M}^{0}+\right.$ $\left.i P_{M}^{0}\right) / \sqrt{2}$. Now using eqs. (6.4), (6.5) and the field decomposition for $\widetilde{\nu}_{i}^{c}$ (mentioned in figure 3 ), it is possible to extract the relevant (concerning figure 3 ) parts of eq. (6.3) as:

$$
\begin{gathered}
\mathcal{L}^{\prime}=\left[-A_{\lambda} \lambda H_{u}^{0} H_{d}^{0} \frac{\left(S_{M}^{0}+i P_{M}^{0}\right)}{\sqrt{2}}+\frac{A_{\kappa} \kappa}{6 \sqrt{2}} \sum_{i=1}^{3}\left\{\left(\mathcal{U}_{i 1} S_{M}^{0}+\mathcal{U}_{i 2} S_{U_{1}}^{0}+\mathcal{U}_{i 3} S_{U_{2}}^{0}\right)^{3}\right.\right. \\
-i\left(\mathcal{U}_{i 1} P_{M}^{0}+\mathcal{U}_{i 2} P_{U_{1}}^{0}+\mathcal{U}_{i 3} P_{U_{2}}^{0}\right)^{3}
\end{gathered}
$$

\footnotetext{
${ }^{21} \operatorname{Our}\left(\widetilde{H}_{d}^{0}\right) \equiv\left(\widetilde{H}_{d}\right)$ of ref. [23].
} 


$$
\begin{gathered}
+3 i\left(\mathcal{U}_{i 1} S_{M}^{0}+\mathcal{U}_{i 2} S_{U_{1}}^{0}+\mathcal{U}_{i 3} S_{U_{2}}^{0}\right)^{2}\left(\mathcal{U}_{i 1} P_{M}^{0}+\mathcal{U}_{i 2} P_{U_{1}}^{0}+\mathcal{U}_{i 3} P_{U_{2}}^{0}\right) \\
\left.-3\left(\mathcal{U}_{i 1} S_{M}^{0}+\mathcal{U}_{i 2} S_{U_{1}}^{0}+\mathcal{U}_{i 3} S_{U_{2}}^{0}\right)\left(\mathcal{U}_{i 1} P_{M}^{0}+\mathcal{U}_{i 2} P_{U_{1}}^{0}+\mathcal{U}_{i 3} P_{U_{2}}^{0}\right)^{2}\right\} \\
\left.+\frac{\kappa \boldsymbol{\lambda}}{\sqrt{3}} H_{u}^{0^{*}} H_{d}^{0^{*}}\left(\sqrt{6} \nu^{c}\left(S_{M}^{0}+i P_{M}^{0}\right)+\frac{1}{2}\left\{S_{M}^{0^{2}}+S_{U_{1}}^{0^{2}}+S_{U_{2}}^{0^{2}}-P_{M}^{0^{2}}-P_{U_{1}}^{0^{2}}-P_{U_{2}}^{0^{2}}\right\}\right)+\text { H.c. }\right] \\
+\lambda^{2}\left(\left|H_{u}^{0}\right|^{2}+\left|H_{d}^{0}\right|^{2}\right)\left(\sqrt{6} \nu^{c} S_{M}^{0}+\frac{S_{M}^{0^{2}}+P_{M}^{0^{2}}}{2}\right)+\sqrt{2} \kappa^{2} \nu^{c}\left(\mathcal{U}_{i 1} S_{M}^{0}+\mathcal{U}_{i 2} S_{U_{1}}^{0}+\mathcal{U}_{i 3} S_{U_{2}}^{0}\right)^{3} \\
+\sqrt{2} \kappa^{2} \nu^{c}\left(\mathcal{U}_{i 1} S_{M}^{0}+\mathcal{U}_{i 2} S_{U_{1}}^{0}+\mathcal{U}_{i 3} S_{U_{2}}^{0}\right)\left(\mathcal{U}_{i 1} P_{M}^{0}+\mathcal{U}_{i 2} P_{U_{1}}^{0}+\mathcal{U}_{i 3} P_{U_{2}}^{0}\right)^{2} \\
-\frac{1}{2}\left[\sqrt{2} \kappa \nu^{c}\left(\mathcal{U}_{i 1} S_{M}^{0}+\mathcal{U}_{i 2} S_{U_{1}}^{0}+\mathcal{U}_{i 3} S_{U_{2}}^{0}\right)\left(\mathcal{U}_{i 1} \widetilde{\chi}_{M}^{0}+\mathcal{U}_{i 2} \widetilde{\chi}_{U_{1}}^{0}+\mathcal{U}_{i 3} \widetilde{\chi}_{U_{2}}^{0}\right)^{2}+\text { H.c. }\right] \\
-\frac{1}{2}\left[\sqrt{2} i \kappa \nu^{c}\left(\mathcal{U}_{i 1} P_{M}^{0}+\mathcal{U}_{i 2} P_{U_{1}}^{0}+\mathcal{U}_{i 3} P_{U_{2}}^{0}\right)\left(\mathcal{U}_{i 1} \widetilde{\chi}_{M}^{0}+\mathcal{U}_{i 2} \widetilde{\chi}_{U_{1}}^{0}+\mathcal{U}_{i 3} \widetilde{\chi}_{U_{2}}^{0}\right)^{2}+\text { H.c. }\right] \\
+\frac{1}{2}\left[\lambda H_{u}^{0} \widetilde{H}_{d}^{0} \widetilde{\chi}_{M}^{0}+\text { H.c. }\right] .
\end{gathered}
$$

Here $\mathcal{U}_{i j}$ represent the elements of the matrix $\mathcal{U}$, shown in eq. (6.5). It is apparent ${ }^{22}$ from eq. (6.6) that processes like $S_{4}^{0} \rightarrow S_{M}^{0} S_{U_{1,2}}^{0}, P_{M}^{0} P_{U_{1,2}}^{0}, \widetilde{\chi}_{M}^{0} \widetilde{\chi}_{U_{1,2}}^{0}$ or $S_{4}^{0} \rightarrow S_{U_{1}}^{0} S_{U_{1}}^{0}$, $S_{4}^{0} \rightarrow \widetilde{\chi}_{U_{2}}^{0} \widetilde{\chi}_{U_{2}}^{0}$, etc., are suppressed compared to $S_{4}^{0} \rightarrow S_{M}^{0} S_{M}^{0}, P_{M}^{0} P_{M}^{0}$ and $S_{4}^{0} \rightarrow \widetilde{\chi}_{M}^{0} \widetilde{\chi}_{M}^{0}$ processes. This is due to the presence of smaller couplings for the former, e.g. powers of $\kappa$, that are estimated to be around $\mathcal{O}\left(10^{-2}\right)$ or smaller in the last section. This conclusion weakens in the limit of $\boldsymbol{\lambda} \gtrsim 0.7$, when a coupling like $\boldsymbol{\lambda} \kappa$ appears to be $\mathcal{O}\left(10^{-1}\right)$. Thus, for small to moderate $\boldsymbol{\lambda}$ values as well as for some regions of moderate to large $\boldsymbol{\lambda}$ values, one can use $\sum \operatorname{Br}\left(S_{4}^{0} \rightarrow S_{i}^{0} S_{j}^{0}\right) \approx \operatorname{Br}\left(S_{4}^{0} \rightarrow S_{M}^{0} S_{M}^{0}\right)$. This statement holds true for $P^{0}$ and $\widetilde{\chi}^{0}$ also and has been verified numerically. Further, we have checked numerically that our observations remain valid even when small splittings exist within $\kappa_{i}$ (see eq. (5.3)) values. Furthermore, the relative sign difference for $S^{0}$ and $P^{0}$ normally predicts $\operatorname{Br}\left(S_{4}^{0} \rightarrow\right.$ $\left.S_{M}^{0} S_{M}^{0}\right)>\operatorname{Br}\left(S_{4}^{0} \rightarrow P_{M}^{0} P_{M}^{0}\right)$.

Now, looking at figure 3 , one can estimate the effective couplings that control the decays of the SM Higgs-like $S_{4}^{0}$ into $\widetilde{\nu}^{c}$-like $S_{i}^{0}, P_{i}^{0}$ states, which are given as: $\frac{A_{\lambda}^{2} \lambda^{2} v_{u}}{3 m_{s}^{2}}$, $\frac{A_{\lambda} \boldsymbol{\lambda} \kappa v_{u} \mu}{3 \sqrt{3} m_{s}^{2}}, \frac{A_{\lambda} \boldsymbol{\lambda}^{2} v_{d} \mu}{9 m_{s}^{2}}, \frac{\boldsymbol{\lambda}^{2} v_{u}}{3}, \frac{\boldsymbol{\lambda} \kappa v_{d}}{\sqrt{3}}, \frac{A_{\lambda} A_{\kappa} \boldsymbol{\lambda} \kappa v_{d}}{\sqrt{3} m_{s}^{2}}, \frac{A_{\kappa} \kappa^{2} \mu v_{d}}{3 m_{s}^{2}}$ and $\frac{A_{\kappa} \boldsymbol{\lambda} \kappa \mu v_{u}}{3 \sqrt{3} m_{s}^{2}}$. Here $m_{s}^{2}$ represents the scale of $H_{u, d}^{0}$ and $\widetilde{\nu}^{c}$ soft squared masses, and we have used $\mu=\sqrt{3} \lambda \nu^{c}$ with $\lambda_{i}=\lambda=\frac{\lambda}{\sqrt{3}}$. Thus, following our discussion of the last section, if one considers (1) $A_{\lambda} \approx \mu \sim 1 \mathrm{TeV},{ }^{23}$ (2) $\kappa \sim 10^{-2}$, (3) $v_{u}>v_{d}$ for $\tan \beta>1$, (4) maximum of $A_{\kappa} \sim 125 \mathrm{GeV}$ (see eq. (5.9)) and (5) fixed $m_{s}$ at $1 \mathrm{TeV}$, then the leading coupling goes as $\frac{\boldsymbol{\lambda}^{2} v_{u}}{3} \mathrm{GeV}$. In the same fashion, out of $\frac{\boldsymbol{\lambda}^{2} v_{u}}{3 \mu}, \frac{A_{\lambda} \boldsymbol{\lambda} \kappa v_{d}}{\sqrt{3} m_{s}^{2}}, \frac{\kappa^{2} \mu v_{d}}{3 m_{s}^{2}}$ and $\frac{\boldsymbol{\lambda} \kappa \mu v_{u}}{3 \sqrt{3} m_{s}^{2}}$, the leading coupling that controls the SM Higgs-like $S_{4}^{0}$ decays into right-handed neutrino-like $\widetilde{\chi}_{i+3}^{0} \widetilde{\chi}_{j+3}^{0}$ is given by $\frac{\lambda^{2} v_{u}}{3 \mu}$. Here the parameter $\mu$ has been used to represent the $\widetilde{H}_{d}^{0}$ mass scale.

\footnotetext{
${ }^{22}$ In the last line of eq. (6.6) we have kept things at the level of two-component spinors.

${ }^{23}$ Note that $\mu \approx 100 \mathrm{GeV}$, that holds true for small to moderate $\boldsymbol{\lambda}$ region, reduces the $2^{\text {nd }}$ and $3^{\text {rd }}$ terms further.
} 
With these couplings, assuming $\mathcal{F}\left(m_{S_{4}^{0}}^{2}, m_{X_{i}^{0}}^{2}, m_{X_{j}^{0}}^{2}\right) \approx 1$ in eq. (6.1), the maximum ${ }^{24}$ approximate leading decay widths for $S_{4}^{0} \rightarrow S_{i}^{0} S_{j}^{0}$ and $P_{i}^{0} P_{j}^{0}, \widetilde{\chi}_{i+3}^{0} \widetilde{\chi}_{j+3}^{0}$ processes are then given as

$$
\begin{aligned}
\Gamma_{S_{4}^{0} \rightarrow S_{i}^{0} S_{j}^{0}, P_{i}^{0} P_{j}^{0}} & \approx \frac{\lambda^{4} v^{2} \tan ^{2} \beta}{9\left(1+\tan ^{2} \beta\right)} \times \frac{1}{16 \pi m_{S_{4}^{0}}\left(1+\delta_{i j}\right)} \approx \frac{0.5 \boldsymbol{\lambda}^{4} \tan ^{2} \beta}{\left(1+\tan ^{2} \beta\right)\left(1+\delta_{i j}\right)} \mathrm{GeV} \\
\Gamma_{S_{4}^{0} \rightarrow \widetilde{\chi}_{i+3}^{0} \widetilde{\chi}_{j+3}^{0}} & \approx \frac{\lambda^{4} v^{2} \tan ^{2} \beta}{9 \mu^{2}\left(1+\tan ^{2} \beta\right)}\left[m_{S_{4}^{0}}^{2}-\left(m_{\widetilde{\chi}_{i+3}^{0}}+m_{\widetilde{\chi}_{j+3}^{0}}\right)^{2}\right] \times \frac{1}{16 \pi m_{S_{4}^{0}}\left(1+\delta_{i j}\right)} \\
& \approx \frac{8366 \lambda^{4} \tan ^{2} \beta}{\left(1+\tan ^{2} \beta\right)\left(1+\delta_{i j}\right)}\left[1-\left(\frac{m_{\widetilde{\chi}_{i+3}^{0}}}{m_{S_{4}^{0}}}+\frac{m_{\widetilde{\chi}_{j+3}^{0}}}{m_{S_{4}^{0}}}\right)^{2}\right] \frac{\mathrm{GeV}^{3}}{\mu^{2}}
\end{aligned}
$$

Here we have used $v=174 \mathrm{GeV}$ and $m_{S_{4}^{0}}=125 \mathrm{GeV}$. From eq. (6.7) note that at the limit of $\tan \beta \gg 1, S_{4}^{0} \rightarrow S_{i}^{0} S_{j}^{0}, P_{i}^{0} P_{j}^{0}, \widetilde{\chi}_{i+3}^{0} \widetilde{\chi}_{j+3}^{0}$ decays become independent of $\tan \beta$.

With the formulae as shown in eq. (6.7), one can estimate the relative importance of the new decays, namely $S_{4}^{0} \rightarrow S_{i}^{0} S_{j}^{0}, P_{i}^{0} P_{j}^{0}, \widetilde{\chi}_{i+3}^{0} \widetilde{\chi}_{j+3}^{0}$ with respect to the known and five wellmeasured SM decay modes, namely $S_{4}^{0} \rightarrow b \bar{b}, \tau^{+} \tau^{-}, \gamma \gamma, W^{ \pm} W^{\mp *}$ and $Z Z^{*}[12,17,19,217]$. The branching ratios into these modes and the total decay widths for the SM Higgs boson, from theoretical analyses, are given in refs. $[218,219]$ assuming a huge variation in Higgs mass, $80 \mathrm{GeV}-1000 \mathrm{GeV}$. The total decay width for the SM Higgs boson with a mass of $125 \mathrm{GeV}$ is $\Gamma_{\text {tot }}^{\mathrm{SM}}=4.07_{-0.160}^{+0.162} \mathrm{MeV}[218]$.

It is now essential to discuss the various measured experimental constraints on the SM Higgs-like $S_{4}^{0}$ that are relevant for the discussion of this section. The stringent set of constraints are coming from the measured reduced signal strengths over the five aforesaid SM decay modes. The reduced signal strength, when an on-shell $S_{4}^{0}$ decays into a pair of $X$ particles, $\mu_{X X}\left(S_{4}^{0}\right)$ is given by

$$
\begin{aligned}
\mu_{X X}\left(S_{4}^{0}\right) & =\frac{\sigma_{\text {prod }}\left(S_{4}^{0}\right) \times B r\left(S_{4}^{0} \rightarrow X X\right)}{\sigma_{\text {prod }}\left(h_{\mathrm{SM}}^{0}\right) \times B r\left(h_{\mathrm{SM}}^{0} \rightarrow X X\right)} \\
& =\frac{\sigma_{\text {prod }}\left(S_{4}^{0}\right)}{\sigma_{\text {prod }}\left(h_{\mathrm{SM}}^{0}\right)} \times \frac{\Gamma_{S_{4}^{0} \rightarrow X X}}{\Gamma_{h_{\mathrm{SM}}^{0} \rightarrow X X}} \times \frac{\Gamma_{\mathrm{tot}}^{\mathrm{SM}}}{\Gamma_{\mathrm{tot}}^{\mathrm{SM}^{\prime}}+\Gamma_{\mathrm{tot}}^{\mathrm{NP}}} .
\end{aligned}
$$

Here $h_{\mathrm{SM}}^{0}$ denotes the SM Higgs boson, $\sigma_{\text {prod }}\left(S_{4}^{0}\right)$ and $\sigma_{\text {prod }}\left(h_{\mathrm{SM}}^{0}\right)$ represent the production cross-section of the $S_{4}^{0}$ and $h_{\mathrm{SM}}^{0}$, respectively. We use $\Gamma_{S_{4}^{0} \rightarrow X X}$ and $\Gamma_{h_{\mathrm{SM}}^{0} \rightarrow X X}$ to represent Higgs $\rightarrow X X$ decay width in the new physics (NP) theory (in this case the $\mu \nu \mathrm{SSM}$ ) and in the SM, respectively. The total decay width for the NP is written as a sum of the pure NP decay width $\left(\Gamma_{\mathrm{tot}}^{\mathrm{NP}}\right)$ and that of the SM modes in NP theory $\left(\Gamma_{\mathrm{tot}}^{\mathrm{SM}^{\prime}}\right)$. The quantity $\Gamma_{\mathrm{tot}}^{\mathrm{NP}}$, following eq. (6.7) is written as

$$
\Gamma_{\mathrm{tot}}^{\mathrm{NP}}=\sum_{i, j=1}^{3} \frac{1+\delta_{i j}}{2}\left(\Gamma_{S_{4}^{0} \rightarrow S_{i}^{0} S_{j}^{0}}+\Gamma_{S_{4}^{0} \rightarrow P_{i}^{0} P_{j}^{0}}+\Gamma_{S_{4}^{0} \rightarrow \widetilde{\chi}_{i+3}^{0} \widetilde{\chi}_{j+3}^{0}}\right) .
$$

The latest measured $\mu_{X X}\left(S_{4}^{0}\right)$ values for $X=b, \tau, \gamma, W^{ \pm}$and $Z$ are given in table 1 .

\footnotetext{
${ }^{24}$ With heavier $S_{i}^{0}, P_{i}^{0}$ and $\widetilde{\chi}_{i+3}^{0}$ states, these $\mathcal{F}$ functions reduce further and thereby justify the maximum estimate of these decay widths as shown in eq. (6.7).
} 


\begin{tabular}{|c|c|c|c|}
\cline { 3 - 4 } \multicolumn{1}{c|}{} & Measured value & $m_{S_{4}^{0}}(\mathrm{GeV})$ \\
\hline \multirow{5}{*}{ ATLAS } & $\mu_{b \bar{b}}\left(S_{4}^{0}\right)$ & $0.2_{-0.6}^{+0.7}[12]$ & 125.5 \\
\cline { 2 - 4 } & $\mu_{\tau^{+} \tau^{-}}\left(S_{4}^{0}\right)$ & $1.4_{-0.4}^{+0.5}[12]$ & 125.5 \\
\cline { 2 - 4 } & $\mu_{\gamma \gamma}\left(S_{4}^{0}\right)$ & $1.17 \pm 0.27[19]$ & 125.4 \\
\cline { 2 - 4 } & $\mu_{W W^{*}}\left(S_{4}^{0}\right)$ & $1.0_{-0.29}^{+0.32}[12]$ & 125.5 \\
\cline { 2 - 4 } & $\mu_{Z Z^{*}}\left(S_{4}^{0}\right)$ & $1.44_{-0.33}^{+0.40}[217]$ & 125.36 \\
\cline { 2 - 4 } & Combined & $1.30_{-0.17}^{+0.18}[12]$ & 125.5 \\
\hline \multirow{5}{*}{ CMS } & $\mu_{b \bar{b}}\left(S_{4}^{0}\right)$ & $0.93 \pm 0.49[17]$ & 125 \\
\cline { 2 - 4 } & $\mu_{\tau^{+} \tau^{-}}\left(S_{4}^{0}\right)$ & $0.91 \pm 0.27[17]$ & 125 \\
\cline { 2 - 4 } & $\mu_{\gamma \gamma}\left(S_{4}^{0}\right)$ & $1.13 \pm 0.24[17]$ & 125 \\
\cline { 2 - 4 } & $\mu_{W W^{*}}\left(S_{4}^{0}\right)$ & $0.83 \pm 0.21[17]$ & 125 \\
\cline { 2 - 4 } & $\mu_{Z Z^{*}}\left(S_{4}^{0}\right)$ & $1.00 \pm 0.29[17]$ & 125 \\
\cline { 2 - 4 } & Combined & $1.00 \pm 0.13[17]$ & 125 \\
\hline
\end{tabular}

Table 1. The measured signal strengths up to the $1 \sigma$ errors from the ATLAS and CMS measurements, with the concerned values of Higgs mass. For the ATLAS measurements, $\mu_{b \bar{b}}\left(S_{4}^{0}\right), \mu_{W W^{*}}\left(S_{4}^{0}\right)$ correspond to a combined data set of $\mathrm{E}_{\mathrm{CM}}=7 \mathrm{TeV}$ with $\mathcal{L}=4.6-4.8 \mathrm{fb}^{-1}$ and $\mathrm{E}_{\mathrm{CM}}=8 \mathrm{TeV}$ with $\mathcal{L}=20.3 \mathrm{fb}^{-1}$ [12]. Only $8 \mathrm{TeV}$ data set has been used [12] to evaluate $\mu_{\tau^{+} \tau^{-}}\left(S_{4}^{0}\right)$. In the measurements of $\mu_{\gamma \gamma}\left(S_{4}^{0}\right), \mu_{Z Z^{*}}\left(S_{4}^{0}\right)$, the ATLAS collaboration has used a data set corresponds to $\mathrm{E}_{\mathrm{CM}}=7$ with $\mathcal{L}=4.5 \mathrm{fb}^{-1}$ combined with $\mathrm{E}_{\mathrm{CM}}=8 \mathrm{TeV}$ with $\mathcal{L}=20.3 \mathrm{fb}^{-1}[19,217]$. A data set corresponds to $\mathrm{E}_{\mathrm{CM}}=7$ with $\mathcal{L}=5.1 \mathrm{fb}^{-1}$ and $\mathrm{E}_{\mathrm{CM}}=8 \mathrm{TeV}$ with $\mathcal{L}=19.7 \mathrm{fb}^{-1}$ has been used for the CMS analyses [17].

Additional constraints can appear from the other measurements, e.g. the total decay width $\Gamma_{\text {tot }}=\Gamma_{\text {tot }}^{\mathrm{NP}}+\Gamma_{\text {tot }}^{\mathrm{SM}^{\prime}}$, room for the invisible/non-standard branching fractions, etc. For the former, the concerned CMS limit is $\Gamma_{\text {tot }}<22 \mathrm{MeV}[128,130]$ assuming $m_{S_{4}^{0}}=125.6 \mathrm{GeV}$. The other constraint, i.e. the experimentally allowed window for the invisible/non-standard $S_{4}^{0}$ decay branching fraction at $95 \%$ C. L. is $<0.41$ from the ATLAS [12] while $<0.58$ from the CMS [147] observation.

At the LHC, $g g \rightarrow S_{4}^{0}$ is the leading source of Higgs production. Assuming stops above $1 \mathrm{TeV}, g g \rightarrow S_{4}^{0}$ process in the NP occurs mainly through the top loop, just like the SM. The only difference appears from the concerned coupling, through which a SM-like $S_{4}^{0}$ couples to $t \bar{t}$ in NP. In one line, the ratio of the decay widths for a Higgs-like scalar decaying into $X X$ final state, in the NP and in the SM, is proportional to the ratio of the respective squared couplings. Hence, one gets

$$
\frac{\Gamma_{S_{4}^{0} \rightarrow X X}}{\Gamma_{h_{\mathrm{SM}}^{0} \rightarrow X X}}=\frac{\mathcal{G}_{S_{4}^{0} X X}^{2}}{\mathcal{G}_{h_{\mathrm{SM}}^{0} X X}^{2}},
$$

where $\mathcal{G}$ represents the relevant coupling. The ratios of the relevant squared couplings are 


\begin{tabular}{|c|c|c|c|c|c|c|c|c|c|c|}
\hline$h_{\mathrm{SM}}^{0} \rightarrow m m$ & $\gamma \gamma$ & $Z \gamma$ & $g g$ & $c \bar{c}$ & $b \bar{b}$ & $s \bar{s}$ & $\tau^{+} \tau^{-}$ & $\mu^{+} \mu^{-}$ & $W W^{*}$ & $Z Z^{*}$ \\
\hline $\begin{array}{c}\Gamma_{h_{\mathrm{SM}}^{0} \rightarrow m m} \\
(\mathrm{MeV})\end{array}$ & 0.009 & 0.006 & 0.349 & 0.118 & 2.348 & 0.001 & 0.257 & 0.001 & 0.875 & 0.107 \\
\hline
\end{tabular}

Table 2. Theoretical decay widths for a $125 \mathrm{GeV} h_{\mathrm{SM}}^{0}$ with $\Gamma_{\mathrm{tot}}^{\mathrm{SM}}=4.07 \mathrm{MeV}$, as given in ref. [218]. The corresponding errors are not shown.

given as

$$
\begin{aligned}
\frac{\mathcal{G}_{S_{4}^{0} t \bar{t}}^{2}}{\mathcal{G}_{h_{\mathrm{SM}}^{0} t \bar{t}}^{2}} & =\frac{\left(1+\tan ^{2} \beta\right)}{\tan ^{2} \beta}\left|R_{42}^{S^{0}}\right|^{2}, \quad \frac{\mathcal{G}_{S_{4}^{0} b \bar{b}}^{2}}{\mathcal{G}_{h_{\mathrm{SM}}^{0} b \bar{b}}^{2}}=\frac{\mathcal{G}_{S_{4}^{0} \tau^{+} \tau^{-}}^{2}}{\mathcal{G}_{h_{\mathrm{SM}}^{0} \tau^{+} \tau^{-}}^{2}}=\left(1+\tan ^{2} \beta\right)\left|R_{41}^{S^{0}}\right|^{2}, \\
\frac{\mathcal{G}_{S_{4}^{0} W W}^{2}}{\mathcal{G}_{h_{\mathrm{SM}}^{0} W W}^{2}} & =\frac{\mathcal{G}_{S_{4}^{0} Z Z}^{2}}{\mathcal{G}_{h_{\mathrm{SM}}^{0} Z Z}^{2} Z}=\left|\cos \beta R_{41}^{S^{0}}+\sin \beta R_{42}^{S^{0}}+\frac{\nu_{i}}{v} R_{4, i+5}^{S^{0}}\right|^{2} \approx \frac{\tan ^{2} \beta}{\left(1+\tan ^{2} \beta\right)}\left|\frac{R_{41}^{S^{0}}}{\tan \beta}+R_{42}^{S^{0}}\right|^{2}, \\
\frac{\mathcal{G}_{S_{4}^{0} \gamma \gamma}^{2}}{\mathcal{G}_{h_{\mathrm{SM}}^{0} \gamma \gamma}^{2}} & \approx \frac{\left(1+\tan ^{2} \beta\right)}{\tan ^{2} \beta}\left|R_{42}^{S^{0}}\right|^{2} .
\end{aligned}
$$

Here we have used $v_{u}=v \sin \beta, v_{d}=v \cos \beta$ and $\nu_{i} / v \sim \mathcal{O}\left(10^{-6}\right) \ll 1$. The couplings $R_{41}^{S^{0}}, R_{42}^{S^{0}}, R_{4, i+5}^{S^{0}}$, following ref. [23], are given in ref. [32]. These are related to the composition of $H_{d}^{0}, H_{u}^{0}$ and left-handed sneutrinos in $S_{4}^{0}$ with the maximum possible squared value equal to 1 . Thus, neglecting $\left(\nu_{i} / v\right) R_{4, i+5}^{S^{0}}$ in the last line of eq. (6.11) is well justified. In the derivation of $\mathcal{G}_{S_{4}^{0} \gamma \gamma}^{2} / \mathcal{G}_{h_{\mathrm{SM}}^{0} \gamma \gamma}^{2}$, we have assumed that the primary contribution to the SM-like $S_{4}^{0} \rightarrow \gamma \gamma$ emerges through the top loop, similar to the SM. The latter is well motivated in the absence of light charged SUSY particles.

It is now possible to use eqs. (6.10) and (6.11) to write $\Gamma_{\text {tot }}^{\mathrm{SM}^{\prime}}$ as

$$
\begin{aligned}
\Gamma_{\mathrm{tot}}^{\mathrm{SM}^{\prime}}= & \sum_{m}\left(\frac{\mathcal{G}_{S_{4}^{0} m m}^{2}}{\mathcal{G}_{h_{\mathrm{SM}}^{0} m m}^{2}}\right) \times \Gamma_{h_{\mathrm{SM}}^{0} \rightarrow m m} \\
\approx & \frac{\left(1+\tan ^{2} \beta\right)}{\tan ^{2} \beta}\left|R_{42}^{S^{0}}\right|^{2}\left(\Gamma_{h_{\mathrm{SM}}^{0} \rightarrow \gamma \gamma}+\Gamma_{h_{\mathrm{SM}}^{0} \rightarrow Z \gamma}+\Gamma_{h_{\mathrm{SM}}^{0} \rightarrow g g}+\Gamma_{h_{\mathrm{SM}}^{0} \rightarrow c \bar{c}}\right) \\
& +\left(1+\tan ^{2} \beta\right)\left|R_{41}^{S^{0}}\right|^{2}\left(\Gamma_{h_{\mathrm{SM}}^{0} \rightarrow b \bar{b}}+\Gamma_{h_{\mathrm{SM}}^{0} \rightarrow s \bar{s}}+\Gamma_{h_{\mathrm{SM}}^{0} \rightarrow \tau^{+} \tau^{-}}+\Gamma_{h_{\mathrm{SM}}^{0} \rightarrow \mu^{+} \mu^{-}}\right) \\
& +\frac{\tan ^{2} \beta}{\left(1+\tan ^{2} \beta\right)}\left|\frac{R_{41}^{S^{0}}}{\tan \beta}+R_{42}^{S^{0}}\right|^{2}\left(\Gamma_{h_{\mathrm{SM}}^{0} \rightarrow W W^{*}}+\Gamma_{h_{\mathrm{SM}}^{0} \rightarrow Z Z^{*}}\right)
\end{aligned}
$$

where the sum exists over all the known SM modes. Here we have used the fact that $c \bar{c}$ and $s \bar{s}, \mu^{+} \mu^{-}$couples to the $S_{4}^{0}$ like $t \bar{t}$ and $b \bar{b}$, respectively. We also assume that the leading source of $S_{4}^{0} \rightarrow Z \gamma$ process is the top loop. One can rewrite eq. (6.12) using the decay widths for a $125 \mathrm{GeV} h_{\mathrm{SM}}^{0}$ into different modes as given in table 2 . The result is given by

$$
\begin{aligned}
\Gamma_{\text {tot }}^{\mathrm{SM}^{\prime}}(\mathrm{MeV}) \approx & \frac{\left(1+\tan ^{2} \beta\right)}{\tan ^{2} \beta}\left|R_{42}^{S^{0}}\right|^{2} \times 0.48+\left(1+\tan ^{2} \beta\right)\left|R_{41}^{S^{0}}\right|^{2} \times 2.61 \\
& +\frac{\tan ^{2} \beta}{\left(1+\tan ^{2} \beta\right)}\left|\frac{R_{41}^{S^{0}}}{\tan \beta}+R_{42}^{S^{0}}\right|^{2} \times 0.98
\end{aligned}
$$


In the light of these discussions, together with eqs. (6.10) and (6.11), one can reinterpret eq. (6.8) as

$$
\mu_{X X}\left(S_{4}^{0}\right) \approx \frac{\mathcal{G}_{S_{4}^{0} t \bar{t}}^{2}}{\mathcal{G}_{h_{\mathrm{SM}}^{0} t \bar{t}}^{2}} \times \frac{\mathcal{G}_{S_{4}^{0} X X}^{2}}{\mathcal{G}_{h_{\mathrm{SM}}^{0}}^{2} X X} \times \frac{\Gamma_{\mathrm{tot}}^{\mathrm{SM}}}{\left(\Gamma_{\mathrm{tot}}^{\mathrm{SM}}+\Gamma_{\mathrm{tot}}^{\mathrm{NP}}\right)},
$$

and consequently,

$$
\begin{gathered}
\mu_{\gamma \gamma}\left(S_{4}^{0}\right) \approx \frac{\left(1+\tan ^{2} \beta\right)^{2}}{\tan ^{4} \beta}\left|R_{42}^{S^{0}}\right|^{4} \times \frac{4.07 \mathrm{MeV}}{\left(\Gamma_{\mathrm{tot}}^{\mathrm{SM}}+\Gamma_{\mathrm{tot}}^{\mathrm{NP}}\right)}, \\
\mu_{b \bar{b}}\left(S_{4}^{0}\right), \mu_{\tau^{+} \tau^{-}}\left(S_{4}^{0}\right) \approx \frac{\left(1+\tan ^{2} \beta\right)^{2}}{\tan ^{2} \beta}\left|R_{41}^{S^{0}}\right|^{2}\left|R_{42}^{S^{0}}\right|^{2} \times \frac{4.07 \mathrm{MeV}}{\left(\Gamma_{\mathrm{tot}}^{\mathrm{SM}^{\prime}}+\Gamma_{\mathrm{tot}}^{\mathrm{NP}}\right)}, \\
\mu_{W W^{*}}\left(S_{4}^{0}\right), \mu_{Z Z^{*}}\left(S_{4}^{0}\right) \approx\left|\frac{R_{41}^{S^{0}} R_{42}^{S^{0}}}{\tan \beta}+R_{42}^{S^{02}}\right|^{2} \times \frac{4.07 \mathrm{MeV}}{\left(\Gamma_{\mathrm{tot}}^{\mathrm{SM}}+\Gamma_{\mathrm{tot}}^{\mathrm{NP}}\right)} .
\end{gathered}
$$

Here we have used $\Gamma_{\text {tot }}^{\mathrm{SM}}=4.07 \mathrm{MeV}$ for $m_{h_{\mathrm{SM}}^{0}}=125 \mathrm{GeV}$ and the units of $\Gamma_{\text {tot }}^{\mathrm{SM}^{\prime}}$ and $\Gamma_{\mathrm{tot}}^{\mathrm{NP}}$ are given in MeV. Expressions for $\Gamma_{\text {tot }}^{\mathrm{NP}}$ and $\Gamma_{\text {tot }}^{\mathrm{SM}^{\prime}}$ are given in eqs. (6.7), (6.9) and eq. (6.13), respectively.

Let us consider now, as an example, ${ }^{25}\left|R_{42}^{S^{0}}\right|^{2} \cong 0.9,\left|R_{41}^{S^{0}}\right|^{2} \cong 0.1$ and negligible $\widetilde{\nu}^{c}$ composition in $S_{4}^{0}$. These numbers will be used henceforth. Note that $\left(1+\tan ^{2} \beta\right) / \tan ^{2} \beta$ varies from 1.25 to 1 as $\tan \beta$ changes from 2 to very large values. On the other hand, $(1+$ $\tan ^{2} \beta$ ) grows very fast with $\tan \beta$. Hence, a small $\left|R_{41}^{S^{0}}\right|^{2}$ is essential to accommodate $\Gamma_{\text {tot }}^{\mathrm{SM}^{\prime}}$ as well as $\mu_{b \bar{b}}\left(S_{4}^{0}\right), \mu_{\tau^{+} \tau^{-}}\left(S_{4}^{0}\right)$ in an experimentally allowed way $[12,17,19,128,130,217]$. An alternate way to reduce $\left|R_{41}^{S^{0}}\right|^{2}$ and hence $\Gamma_{\text {tot }}^{S M^{\prime}}$, and consequently $\mu_{b \bar{b}}\left(S_{4}^{0}\right), \mu_{\tau^{+} \tau^{-}}\left(S_{4}^{0}\right)$, with respect to the $\mathrm{SM}$, is to introduce more singlet component in $S_{4}^{0}$. This procedure, however, is valid for small $\tan \beta$ values, unless one considers $\left|R_{41}^{S^{0}}\right|^{2} \rightarrow 0$. In this way one can also increase $\mu_{\gamma \gamma}\left(S_{4}^{0}\right)[220,221]$. For the latter, the existence of light charged SUSY particles (e.g., stau, chargino) in the spectrum [222-225] is another possibility.

The choice of $\left|R_{41}^{S^{0}}\right|^{2}=0.1$ and $^{26} \tan \beta \lesssim 3$, using eq. (6.13) implies $\Gamma_{\text {tot }}^{\mathrm{SM}^{\prime}} \lesssim \Gamma_{\text {tot }}^{\mathrm{SM}}=$ $4.07 \mathrm{MeV}$, and hence $\Gamma_{\text {tot }}^{\mathrm{SM}} /\left(\Gamma_{\text {tot }}^{\mathrm{SM}^{\prime}}+\Gamma_{\mathrm{tot}}^{\mathrm{NP}}\right) \gtrsim 1$, given that $\Gamma_{\text {tot }}^{\mathrm{NP}} \ll \Gamma_{\text {tot }}^{\mathrm{SM}^{\prime}}$, as expected for small to moderate $\boldsymbol{\lambda}$ values (see eqs. (6.7) and (6.9)). This behaviour is well expected since with small $\left|R_{41}^{S^{0}}\right|^{2}$ and small $\tan \beta$ (see eq. (6.13)), the decay widths for all the down-type fermions reduce below their SM values. This reduction, especially for $b \bar{b}$ which is the leading decay mode for the SM-like $S_{4}^{0}$, diminishes $\Gamma_{\text {tot }}^{\mathrm{SM}^{\prime}}$ mainly through a reduction in $\Gamma_{S_{4}^{0} \rightarrow b \bar{b}}$. Using $\left|R_{42}^{S^{0}}\right|^{2}=0.9$ and $\left|R_{41}^{S^{0}}\right|^{2}=0.1$, in the limit of a negligible $\Gamma_{\text {tot }}^{\mathrm{NP}}$, one evaluates from eq. (6.15) that $\mu_{\gamma \gamma}\left(S_{4}^{0}\right), \mu_{W W^{*}}\left(S_{4}^{0}\right), \mu_{Z Z^{*}}\left(S_{4}^{0}\right) \gtrsim 1$ for $\tan \beta \lesssim 3$ while $\mu_{b \bar{b}}\left(S_{4}^{0}\right), \mu_{\tau^{+} \tau^{-}}\left(S_{4}^{0}\right)<1$. This observation has an important consequence, i.e. departure of all the five reduced signal

\footnotetext{
${ }^{25}$ The set of numbers used here, i.e. $\left|R_{41}^{S^{0}}\right|^{2} \cong 0.1$ and $\left|R_{42}^{S^{0}}\right|^{2} \cong 0.9$, is valid mainly for small to moderate $\boldsymbol{\lambda}$ region. For larger $\boldsymbol{\lambda}$ values (e.g., $\boldsymbol{\lambda} \gtrsim 0.3$ ), singlet-doublet mixing is enhanced and consequently a nonnegligible singlet composition $\sum\left|R_{4, i+3}^{S^{0}}\right|^{2}$ appears in $S_{4}^{0}$. The concerned formulas $((6.13)$ and $(6.15))$, however, remain still valid but with a smaller values of $\left|R_{41}^{S^{0}}\right|^{2}$ and $\left|R_{42}^{S^{0}}\right|^{2}$.

${ }^{26}$ Note that the limit on $\tan \beta$ attains smaller values with increasing $\left|R_{41}^{S^{0}}\right|^{2}$, e.g. $\tan \beta \lesssim 1.9$ for $\left|R_{41}^{S^{0}}\right|^{2}=0.2$.
} 
strengths from the value 1 remains possible even when $\Gamma_{\text {tot }}^{\mathrm{NP}}=0$, i.e. when no additional decay modes exist for $S_{4}^{0}$. This phenomenon, in the context of the $\mu \nu \mathrm{SSM}$ can occur when $m_{S_{i}^{0}, P_{i}^{0}}$ and $m_{\widetilde{\chi}_{i+3}^{0}}$ are larger or comparable to $m_{S_{4}^{0}}$.

Following now the trend of our past analyses, as of the last two sections, we again start with the discussion of the $0.01 \lesssim \boldsymbol{\lambda} \leq 0.1$ scenario.

(a) Small to moderate $\lambda$ : in this region of the parameter space, the maximum value of $\boldsymbol{\lambda}$ is 0.1 . It is thus evident from eq. (6.7) that $S_{4}^{0} \rightarrow S_{i}^{0} S_{j}^{0}, S_{4}^{0} \rightarrow P_{i}^{0} P_{j}^{0}, S_{4}^{0} \rightarrow \widetilde{\chi}_{i+3}^{0} \widetilde{\chi}_{j+3}^{0}$ decays are naturally suppressed in this corner of the parameter space due to the smallness of the $\boldsymbol{\lambda}$ parameter. Following the discussion of the last paragraph, one gets for example with $\tan \beta=2$ and $\mu=100 \mathrm{GeV}, \Gamma_{\text {tot }}^{\mathrm{NP}} \approx 0.036 \mathrm{MeV}$ while $\Gamma_{\text {tot }}^{\mathrm{SM}^{\prime}} \approx 2.81 \mathrm{MeV}$, as evaluated from eqs. (6.9) and (6.13). Hence, numerically one gets $\mu_{\gamma \gamma}\left(S_{4}^{0}\right) \approx 1.81, \mu_{b \bar{b}}\left(S_{4}^{0}\right), \mu_{\tau^{+} \tau^{-}}\left(S_{4}^{0}\right) \approx$ 0.81 and $\mu_{W W^{*}}\left(S_{4}^{0}\right), \mu_{Z Z^{*}}\left(S_{4}^{0}\right) \approx 1.58$. Clearly, from table 1 , for $\gamma \gamma$ one needs to consider at least $3 \sigma$ variation to accommodate this scenario experimentally. The conclusion is also very similar for $W W^{*}$ and $Z Z^{*}$ while for $b \bar{b}$ the number is within the $1 \sigma$ range of the experimental measurements. For $\tau^{+} \tau^{-}$, the number falls within the $1 \sigma$ and $3 \sigma$ range of the CMS and the ATLAS measurements, respectively. For the evaluation of $\Gamma_{\mathrm{tot}}^{\mathrm{NP}}$, we have considered the proper numerical factors as mentioned in the caption of figure 3 , took $i=j$ and $m_{\widetilde{\chi}_{i+3}^{0}}=10 \mathrm{GeV}$. Furthermore, we have used the fact, as already stated, that for this corner of the parameter space $\Gamma_{\mathrm{tot}}^{\mathrm{NP}} \approx \Gamma_{S_{4}^{0} \rightarrow S_{M}^{0} S_{M}^{0}}+\Gamma_{S_{4}^{0} \rightarrow P_{M}^{0} P_{M}^{0}}+\Gamma_{S_{4}^{0} \rightarrow \widetilde{\chi}_{M}^{0} \widetilde{\chi}_{M}^{0}}$.

Taking $\tan \beta=3$ and keeping everything else the same, one gets $\Gamma_{\text {tot }}^{\mathrm{NP}} \approx 0.041 \mathrm{MeV}$, $\Gamma_{\mathrm{tot}}^{\mathrm{SM}^{\prime}} \approx 4.07 \mathrm{MeV}, \mu_{\gamma \gamma}\left(S_{4}^{0}\right), \mu_{b \bar{b}}\left(S_{4}^{0}\right), \mu_{\tau^{+} \tau^{-}}\left(S_{4}^{0}\right), \mu_{W W^{*}}\left(S_{4}^{0}\right), \mu_{Z Z^{*}}\left(S_{4}^{0}\right) \approx 0.99$. These numbers, as evident from table 1 , are within the $1 \sigma$ ranges of the CMS measurements while for some of the cases fall within the respective $2 \sigma$ ranges of the ATLAS measurements. It is thus important to note that even in the presence of a non-vanishing new physics effect, all the five reduced signal strengths can remain very close to 1 , the expected SM value. With larger $\tan \beta, \mu_{b \bar{b}}\left(S_{4}^{0}\right), \mu_{\tau^{+} \tau^{-}}\left(S_{4}^{0}\right)$ enhance while $\mu_{\gamma \gamma}\left(S_{4}^{0}\right), \mu_{W W^{*}}\left(S_{4}^{0}\right), \mu_{Z Z^{*}}\left(S_{4}^{0}\right)$ decrease further. The $\mu_{\gamma \gamma}\left(S_{4}^{0}\right)$ goes beyond the respective $3 \sigma$ range around $\tan \beta \gtrsim 5$. In this corner of the parameter space, using the chosen values of $\left|R_{42}^{S^{0}}\right|^{2}=0.9$ and $\left|R_{41}^{S^{0}}\right|^{2}=0.1$, all of the three $\mu_{\gamma \gamma}\left(S_{4}^{0}\right), \mu_{W W^{*}}\left(S_{4}^{0}\right), \mu_{Z Z^{*}}\left(S_{4}^{0}\right) \mathrm{s}$ remain larger than 1 , but within the respective $2 \sigma$ CMS ranges, for $2.5 \lesssim \tan \beta \lesssim 2.95$. For $\tan \beta>2.98, \mu_{\gamma \gamma}\left(S_{4}^{0}\right), \mu_{W W^{*}}\left(S_{4}^{0}\right)$ and $\mu_{Z Z^{*}}\left(S_{4}^{0}\right)$ reduce below 1. In this region of the parameter space, concerning the CMS measurements (see table 1), all the five reduced signal strengths remain within their respective $2 \sigma$ ranges for $2.5 \lesssim \tan \beta \lesssim 3.9$. With a different choice of $\left|R_{42}^{S^{0}}\right|^{2}$ and $\left|R_{41}^{S^{0}}\right|^{2}$, one gets a shift in the range of $\tan \beta$ towards smaller values. For example, with $\left|R_{42}^{S^{0}}\right|^{2}=0.75$ and $\left|R_{41}^{S^{0}}\right|^{2}=0.25$, one evaluates $1.45 \lesssim \tan \beta \lesssim 2.15$ as the preferred range of $\tan \beta$, where all the five reduced signal strengths lie within their respective CMS $2 \sigma$ ranges (see table 1 ).

In this connection, note that a bino-like lightest neutralino (defined as $\widetilde{\chi}_{b}^{0}$ ) of the same mass, i.e. $m_{\widetilde{\chi}_{i+3}^{0}} \approx m_{\widetilde{\chi}_{b}^{0}}$, can also contribute to the $S_{4}^{0}$ decay phenomenology. In this case the coupling goes as $\frac{g_{1} v_{u}}{\mu}$. Hence, one needs to multiply $\Gamma_{S_{4}^{0} \rightarrow \widetilde{\chi}_{i+3}^{0} \widetilde{\chi}_{i+3}^{0}}$ with $9 g_{1}^{4} / \boldsymbol{\lambda}^{4}$ to get $\Gamma_{S_{4}^{0} \rightarrow \widetilde{\chi}_{b}^{0} \widetilde{\chi}_{b}^{0}}$. With $g_{1} \approx 0.352$ one evaluates $\Gamma_{S_{4}^{0} \rightarrow \widetilde{\chi}_{b}^{0} \widetilde{\chi}_{b}^{0}} \approx 25.34 \mathrm{MeV}$ for $\boldsymbol{\lambda}=0.1$ and $\tan \beta=3$, with the proper numerical factor as mentioned in figure 3 . This gives $\mu_{\gamma \gamma}\left(S_{4}^{0}\right), \mu_{W W^{*}}\left(S_{4}^{0}\right), \mu_{Z Z^{*}}\left(S_{4}^{0}\right) \approx 0.14$ which are beyond/at the boundary of the respective 
$3 \sigma$ ranges as measured from the CMS (see table 1). Further, $\Gamma_{S_{4}^{0} \rightarrow \widetilde{\chi}_{b}^{0} \widetilde{\chi}_{b}^{0}}>22 \mathrm{MeV}$ is also excluded by the CMS decay width measurement $[128,130]$. It is nonetheless possible to accommodate a light $\widetilde{\chi}_{b}^{0}$ with larger $\mu$ values, e.g. $\mu=500 \mathrm{GeV}$ (with $\boldsymbol{\lambda}=0.1$ and $\tan \beta=3)$, that gives $\Gamma_{\mathrm{tot}}^{\mathrm{NP}}=\Gamma_{S_{4}^{0} \rightarrow \widetilde{\chi}_{b}^{0} \widetilde{\chi}_{b}^{0}} \approx 1.01 \mathrm{MeV}, \mu_{\gamma \gamma}\left(S_{4}^{0}\right), \mu_{b \bar{b}}\left(S_{4}^{0}\right), \mu_{\tau^{+} \tau^{-}}\left(S_{4}^{0}\right)$ and $\mu_{W W^{*}}\left(S_{4}^{0}\right), \mu_{Z Z^{*}}\left(S_{4}^{0}\right) \approx 0.80$. All these numbers ${ }^{27}$ are within the $2 \sigma$ measured values as shown in table 1 .

To summarise, depending on $\tan \beta$ values, experimentally allowed new decay channels for the SM Higgs-like $S_{4}^{0}$ into scalars, pseudoscalars and neutralinos are possible with respect to known SM decay modes. Taking into account the results of section 3, we note that: prompt leptons/taus/jets/photons from a singlet-like scalar/pseudoscalar are favourable in the small to moderate $\boldsymbol{\lambda}$ scenario. A similar conclusion holds for the displaced objects from a singlino-like neutralino. On the contrary, with a bino-like lightest neutralino displaced leptons/taus/jets/photons are difficult in this region of the parameter space unless one considers a large $\mu$-value. The associated decay length for a $\widetilde{\chi}_{b}^{0}$, with a mass smaller than $M_{W}$, is normally larger compared to a right-handed neutrino-like $\widetilde{\chi}_{i+3}^{0}$ of the same mass. The reason, as already mentioned in subsection 3.2, is related to the natural feasibility of having lighter right-handed sneutrino-like $S_{i}^{0}, P_{i}^{0}$ states for the latter.

(b) Moderate to large $\lambda$ : in this region of the parameter space we consider two limiting representative scenarios: (1) $\boldsymbol{\lambda}=0.2$ and $\mu=350 \mathrm{GeV}$, and (2) $\boldsymbol{\lambda}=0.7$ and $\mu=1200 \mathrm{GeV}$.

For scenario $1, \mu_{\gamma \gamma}\left(S_{4}^{0}\right), \mu_{W W^{*}}\left(S_{4}^{0}\right), \mu_{W W^{*}}\left(S_{4}^{0}\right)$ remain larger than 1 for $\tan \beta \lesssim 2.75$. The quantities $\mu_{b \bar{b}}\left(S_{4}^{0}\right)$ and $\mu_{\tau^{+} \tau^{-}}\left(S_{4}^{0}\right)$ go above 1 from $\tan \beta \gtrsim 3.60$ when $\mu_{\gamma \gamma}\left(S_{4}^{0}\right)$, $\mu_{W W^{*}}\left(S_{4}^{0}\right), \mu_{Z Z^{*}}\left(S_{4}^{0}\right)$ diminish to $\approx 0.70,0.72$ and 0.72 , respectively. In this region of $\tan \beta$ values, $\Gamma_{\text {tot }}^{\mathrm{SM}^{\prime}}$ wins over $\Gamma_{\mathrm{tot}}^{\mathrm{SM}}$. Concerning the measured values from the CMS (the ATLAS limits are more flexible), as given in table $1, \mu_{\gamma \gamma}\left(S_{4}^{0}\right)$ goes beyond respective $2 \sigma$ range from $\tan \beta \gtrsim 3.75$ while $\mu_{W W^{*}}\left(S_{4}^{0}\right), \mu_{Z Z^{*}}\left(S_{4}^{0}\right)$ attain the same from $\tan \beta \gtrsim 5.15$. The reduced signal strengths for all the five measured modes, with our choice of $\left|R_{42}^{S^{0}}\right|^{2}=0.9$ and $\left|R_{41}^{S^{0}}\right|^{2}=0.1$, remain within their respective $2 \sigma$ CMS ranges for $2.3 \lesssim \tan \beta \lesssim 3.75$. The total decay width $\Gamma_{\text {tot }}^{\mathrm{SM}^{\prime}}+\Gamma_{\text {tot }}^{\mathrm{NP}}$ remains below the CMS limit of $22 \mathrm{MeV}[128,130]$ unless $\tan \beta \gtrsim 8.8$. This region of $\tan \beta$ value, however, is also excluded from $\mu_{\gamma \gamma}\left(S_{4}^{0}\right), \mu_{W W^{*}}\left(S_{4}^{0}\right)$ and $\mu_{Z Z^{*}}\left(S_{4}^{0}\right)$ measurements. In this region, especially as $\boldsymbol{\lambda} \rightarrow 0.7$, additional constraints can appear through the possible $Z \rightarrow S_{i}^{0} P_{j}^{0}, \widetilde{\chi}_{i+3}^{0} \widetilde{\chi}_{j+3}^{0}$ decay modes [51].

Scenario 2, on the contrary, even with $\tan \beta \rightarrow 1$ predicts $\mu_{\gamma \gamma}\left(S_{4}^{0}\right), \mu_{W W^{*}}\left(S_{4}^{0}\right)$, $\mu_{W W^{*}}\left(S_{4}^{0}\right)$ and $\mu_{\tau^{+} \tau^{-}}\left(S_{4}^{0}\right)$ beyond the respective $3 \sigma$ ranges (see table 1 ). Moreover, in this corner of the parameter space, even with $\Gamma_{\text {tot }}^{\mathrm{NP}} \approx \Gamma_{S_{4}^{0} \rightarrow S_{M}^{0} S_{M}^{0}}+\Gamma_{S_{4}^{0} \rightarrow P_{M}^{0} P_{M}^{0}}+\Gamma_{S_{4}^{0} \rightarrow \widetilde{\chi}_{M}^{0} \widetilde{\chi}_{M}^{0}}$, one estimates $\Gamma_{\text {tot }}^{\mathrm{NP}}>22 \mathrm{MeV}$, which is excluded by the CMS measurement $[128,130]$. Such a large $\Gamma_{\text {tot }}^{\mathrm{NP}}$, for $\boldsymbol{\lambda}=0.7$, is capable of giving a $\mu_{b \bar{b}}\left(S_{4}^{0}\right)$ value beyond the $2 \sigma$ range, in spite of the huge associated errors (see table 1). The large contribution appears mainly through $S_{4}^{0} \rightarrow S_{i}^{0} S_{j}^{0}, P_{i}^{0} P_{j}^{0}$ modes, e.g. about $30 \mathrm{MeV}$ for $S_{4}^{0} \rightarrow S_{M}^{0} S_{M}^{0}+P_{M}^{0} P_{M}^{0}$ process. Contribution from $\widetilde{\chi}_{i+3}^{0} \widetilde{\chi}_{j+3}^{0}$ modes remain much suppressed compared to the former, e.g. with $\tan \beta=2$ one gets $\Gamma_{S_{4}^{0} \rightarrow \widetilde{\chi}_{M}^{0} \widetilde{\chi}_{M}^{0}} \approx 0.3 \mathrm{MeV}$ while $S_{4}^{0} \rightarrow S_{M}^{0} S_{M}^{0}$ is $\approx 24 \mathrm{MeV}$. Thus, existence of new $S_{4}^{0}$ decays, especially $S_{4}^{0} \rightarrow S_{i}^{0} S_{j}^{0}, P_{i}^{0} P_{j}^{0}$, are hardly possible in this region.

\footnotetext{
${ }^{27}$ For ATLAS $\tau^{+} \tau^{-}$, one needs to consider a variation in the $3 \sigma$ range.
} 
Further, as already emphasised in section 5, larger singlet-doublet admixture in $S_{i}^{0}, P_{i}^{0}$ and $\widetilde{\chi}_{i+3}^{0}$ as $\boldsymbol{\lambda} \rightarrow 0.7$ makes it harder to accommodate these states in an experimentally allowed way. Nonetheless, for this region of the $\boldsymbol{\lambda}$ value, $S_{i}^{0}, P_{i}^{0}$ and $\widetilde{\chi}_{i+3}^{0}$ states comparable or heavier than $S_{4}^{0}$ remain an allowed possibility. This scenario, as stated earlier in the last paragraph before the discussion of small to moderate $\boldsymbol{\lambda}$ region, can still predict $\mu_{\gamma \gamma}\left(S_{4}^{0}\right), \mu_{W W^{*}}\left(S_{4}^{0}\right), \mu_{Z Z^{*}}\left(S_{4}^{0}\right)>1$ depending on the scale of $\tan \beta$. Note that as $\boldsymbol{\lambda} \rightarrow 0.7$, the severe constraint on the presence of light $S_{i}^{0}, P_{i}^{0}$ states predicts larger decay length for a light $\widetilde{\chi}_{i+3}^{0}$, as already addressed in subsection 3.2.

A $\widetilde{\chi}_{b}^{0}$ of the same mass remains experimentally difficult for scenario 1 for $\tan \beta \gtrsim 3.2$. For example, with $\tan \beta=3.2$ scenario 1 gives $\mu_{\gamma \gamma}\left(S_{4}^{0}\right) \approx 0.61$. All the five measured reduced signal strengths remain within the $2 \sigma$ range for $1.6 \lesssim \tan \beta \lesssim 3.2$. Here we consider only the CMS limits as given by table 1 . On the other hand, a $\widetilde{\chi}_{b}^{0}$ of the same mass remains well possible for scenario 2 , e.g. one gets $\mu_{\gamma \gamma}\left(S_{4}^{0}\right), \mu_{W W^{*}}\left(S_{4}^{0}\right), \mu_{Z Z^{*}}\left(S_{4}^{0}\right) \gtrsim 1$ for $\tan \beta \lesssim 2.9$. Concentrating on the CMS results, one observes that all of the five $\mu_{X X}\left(S_{4}^{0}\right)$ remain within their respective $2 \sigma$ ranges for $2.45 \lesssim \tan \beta \lesssim 3.85$, with the chosen values of $\left|R_{42}^{S^{0}}\right|^{2}$ and $\left|R_{41}^{S^{0}}\right|^{2}$. Clearly, the existence of a light $\widetilde{\chi}_{b}^{0}$ is more feasible for $\boldsymbol{\lambda}=0.7$ and normally $\Gamma_{S_{4}^{0} \rightarrow \widetilde{\chi}_{b}^{0} \widetilde{\chi}_{b}^{0}}>\Gamma_{S_{4}^{0} \rightarrow \widetilde{\chi}_{i+3}^{0} \widetilde{\chi}_{j+3}^{0}}$ up to $\boldsymbol{\lambda} \approx 0.6$. For $0.6 \lesssim \boldsymbol{\lambda} \leq 0.7$, on the contrary, $\Gamma_{S_{4}^{0} \rightarrow \widetilde{\chi}_{i+3}^{0} \widetilde{\chi}_{j+3}^{0}}$

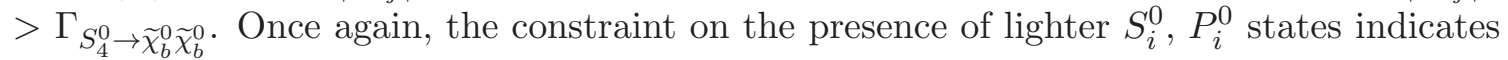
larger decay length as $\boldsymbol{\lambda} \rightarrow 0.7$.

In a nutshell, in this region of the parameter space, depending on the value of $\boldsymbol{\lambda}$ and $\tan \beta$, both prompt and displaced objects are possible from the decays of a singlet-like $S_{i}^{0}, P_{i}^{0}$ and $\widetilde{\chi}_{i+3}^{0}$. Unlike the scalars/pseudoscalars, the neutralino decays, in the absence of a lighter $S_{i}^{0} / P_{i}^{0}$ state, remain possible for the entire span of $\boldsymbol{\lambda}$ values. A $\widetilde{\chi}_{b}^{0}$ is also feasible for larger $\boldsymbol{\lambda}$ values. Enhanced branching fractions, mainly for $S_{4}^{0} \rightarrow S_{i} S_{j}^{0}, P_{i}^{0} P_{j}^{0}$ decay modes, normally put severe constraint on this corner of the parameter space, especially as $\lambda \rightarrow 0.7$. This restriction in turn implies larger decay length for light neutralinos, this time both for singlino-like $\widetilde{\chi}_{i+3}^{0}$ and $\widetilde{\chi}_{b}^{0}$. A detail analysis of $S_{4}^{0} \rightarrow \widetilde{\chi}_{4}^{0} \widetilde{\chi}_{4}^{0}$ decay for this region of the parameter space with $\boldsymbol{\lambda} \approx 0.2$ in the context of the displaced but detectable multi- $\tau$ final state has already been addressed in ref. [50].

(c) Dominant $\lambda$ : the presence of large singlet-doublet mixing, as already stated in section 5, makes it rather hard for the light $S_{i}^{0}, P_{i}^{0}$ states to evade a class of constraints from colliders. Further, large $\boldsymbol{\lambda}$ values normally predict very large decay widths for $S_{4}^{0} \rightarrow S_{i}^{0} S_{j}^{0}, P_{i}^{0} P_{j}^{0}$ processes, that are excluded experimentally. As an example, with $\boldsymbol{\lambda}=1, \mu=1000 \mathrm{GeV}$ one gets $\mu_{\gamma \gamma}\left(S_{4}^{0}\right) \approx 0.1, \mu_{b \bar{b}}\left(S_{4}^{0}\right), \mu_{\tau^{+} \tau^{-}}\left(S_{4}^{0}\right) \approx 0.01$, $\mu_{W W^{*}}\left(S_{4}^{0}\right), \mu_{Z Z^{*}}\left(S_{4}^{0}\right) \approx 0.05$ and $\Gamma_{\text {tot }}^{\mathrm{NP}} \approx 126 \mathrm{MeV}$ even when $\tan \beta \rightarrow 1$. Here, we have assumed that $\Gamma_{\text {tot }}^{\mathrm{NP}} \approx \Gamma_{S_{4}^{0} \rightarrow S_{M}^{0} S_{M}^{0}}+\Gamma_{S_{4}^{0} \rightarrow P_{M}^{0} P_{M}^{0}}+\Gamma_{S_{4}^{0} \rightarrow \widetilde{\chi}_{M}^{0} \widetilde{\chi}_{M}^{0}}$, which however, as already mentioned, gives suppressed contribution compared to the true value for $\boldsymbol{\lambda} \gtrsim 0.7$. Nonetheless, even with this suppressed approximation we observe that all the reduced signal strengths, excluding $\mu_{b \bar{b}}\left(S_{4}^{0}\right)$, are beyond their respective $3 \sigma$ ranges, as shown in table 1. Moreover, the estimated decay width is $\gg 22 \mathrm{MeV}$ and hence is excluded by the CMS result $[128,130]$. For $\widetilde{\chi}_{i+3}^{0}$, on the contrary, the corresponding decay widths remain experimentally viable, provided that the lighter $S_{i}^{0}, P_{i}^{0}$ states are absent. For example, 
$\boldsymbol{\lambda}=1, \mu=1 \mathrm{TeV}$ and $\tan \beta \rightarrow 1$ give $\Gamma_{S_{4}^{0} \rightarrow \widetilde{\chi}_{M}^{0} \widetilde{\chi}_{M}^{0}} \approx 1 \mathrm{MeV}$ while $\Gamma_{S_{4}^{0} \rightarrow S_{M}^{0} S_{M}^{0}, P_{M}^{0} P_{M}^{0}}$ is about $62.5 \mathrm{MeV}$. One must note that in this corner of the parameter space, similar to moderate to large $\boldsymbol{\lambda}$ region with $\boldsymbol{\lambda} \rightarrow 0.7$, one also needs to consider the other non-negligible contributions like $S_{4}^{0} \rightarrow \widetilde{\chi}_{U_{1}}^{0} \widetilde{\chi}_{U_{1}}^{0}, \widetilde{\chi}_{U_{2}}^{0} \widetilde{\chi}_{U_{2}}^{0}, S_{U_{1}}^{0} S_{U_{1}}^{0}, P_{U_{2}}^{0} P_{U_{2}}^{0}$ etc. Combining all these modes for $\widetilde{\chi}_{i+3}^{0}$ and assuming a similar contribution from all of them, the reduced signal strengths, apart from $\mu_{b \bar{b}}\left(S_{4}^{0}\right)$ and $\mu_{\tau^{+} \tau^{-}}\left(S_{4}^{0}\right)$, remain beyond their measured $2 \sigma$ variations for $\tan \beta \gtrsim 1.3$ and, moves to lower values for larger $\boldsymbol{\lambda}$ values. It is thus apparent that the presence of $S_{i}^{0}, P_{i}^{0}$ and $\widetilde{\chi}_{i+3}^{0}$ states, lighter than $m_{S_{4}^{0}} / 2$, is experimentally unrealistic in this region of the parameter space.

Repeating the same exercise with $\mu=1 \mathrm{TeV}$ for a $\widetilde{\chi}_{b}^{0}$ of the same mass, in the absence of lighter $S_{i}^{0}, P_{i}^{0}$ states, one gets all the reduced signal strengths within their respective $2 \sigma$ CMS ranges for $2.4 \lesssim \tan \beta \lesssim 3.8$. For $\mu_{\gamma \gamma}\left(S_{4}^{0}\right), \mu_{W W^{*}}\left(S_{4}^{0}\right), \mu_{Z Z^{*}}\left(S_{4}^{0}\right)$, it remain possible to get $>1$ values, within the respective $2 \sigma$ ranges, in the span of $2.4 \lesssim \tan \beta \lesssim 2.85$. For larger $\mu$ values, that are anticipated with larger $\boldsymbol{\lambda}$ values keeping $\nu^{c}$ fixed at $1 \mathrm{TeV}$, one observes a slight shifting of the aforementioned $\tan \beta$ window towards larger values. $\Gamma_{S_{4} \rightarrow \widetilde{\chi}_{b}^{0} \widetilde{x}_{b}^{0}}$ remains « compared to the CMS upper bound of $22 \mathrm{MeV}$, unless one considers $\tan \beta \gg 1$ which is already excluded from the measured reduced signal strengths.

In summary, a large amount of doublet contamination and very high $\Gamma_{S_{4}^{0} \rightarrow S_{i}^{0} S_{j}^{0}}, \Gamma_{S_{4}^{0} \rightarrow P_{i}^{0} P_{j}^{0}}$ make it hard for the light $S_{i}^{0}, P_{i}^{0}$ states to survive a class of collider constraints for this region of $\boldsymbol{\lambda}$ values. Consequently, the chance of getting an enhancement in the number of prompt final states, from $S_{4}^{0}$ decays, is hardly possible for this corner of the parameter space. The situation is identical for light $\widetilde{\chi}_{i+3}^{0}$, however, in this case the severe constraints appear through the measured reduced signal strengths and not from the decay width $\Gamma_{S_{4}^{0} \rightarrow \widetilde{\chi}_{i+3}^{0} \widetilde{\chi}_{j+3}^{0}}$. A light $\widetilde{\chi}_{b}^{0}$, on the contrary, depending on $\tan \beta$ remains well possible in this span of $\boldsymbol{\lambda}$ values, given that no lighter $S_{i}^{0}, P_{i}^{0}$ state exists in the spectrum. Thus, the associated decay length would appear longer, especially for $m_{\widetilde{\chi}_{b}^{0}}<40 \mathrm{GeV}$ [30], as already mentioned in subsection 3.2. We note in passing, as also stated in the context of moderate to large $\boldsymbol{\lambda}$ values as $\boldsymbol{\lambda} \rightarrow 0.7$, that the absence of light states in the spectrum, e.g. $S_{i}^{0}, P_{i}^{0}$ or $\tilde{\chi}_{i+3}^{0}$, does not exclude the possibility of getting $\mu_{\gamma \gamma}\left(S_{4}^{0}\right), \mu_{W W^{*}}\left(S_{4}^{0}\right), \mu_{Z Z^{*}}\left(S_{4}^{0}\right)>1$ depending on the scale of $\tan \beta$ and the values of $\left|R_{41}^{S^{0}}\right|^{2}$ and $\left|R_{42}^{S^{0}}\right|^{2}$.

\section{Conclusions}

In this work, in the context of the $\mu \nu \mathrm{SSM}$, we have performed an analytical estimate of all the new two-body decays for the SM-like Higgs boson $\left(S_{4}^{0}\right)$, with a mass about $125 \mathrm{GeV}$, in the presence of light singlet-like scalars $\left(S_{i}^{0}\right)$, pseudoscalars $\left(P_{i}^{0}\right)$ and neutralinos $\left(\widetilde{\chi}_{i+3}^{0}\right), i=$ $1,2,3$. We further explored the relative importance of the different parameters not only in the context of new $S_{4}^{0}$ decay modes, but also to accommodate a $125 \mathrm{GeV}$ doublet-like scalar with properties similar to that of the SM-like Higgs boson. At the same time we have identified the singlet-doublet mixing parameters, namely $\lambda_{i}\left(\epsilon_{a b} \lambda_{i} \hat{\nu}_{i}^{c} \hat{H}_{d}^{a} \hat{H}_{u}^{b}\right.$ term in eq. (2.1)), as the key parameters for this analysis since they are very crucial in determining the relative size of those new $S_{4}^{0}$ decay branching fractions compared to that of the SM decay modes. The $\lambda_{i}$-parameters, as explored in section 4 , also produce an extra contribution 
(eq. (4.2)) for the tree-level lightest doublet-like scalar mass which is prominent for low $\tan \beta$. Our discussion is illustrated over the three different regions of the $\lambda_{i}(\equiv \boldsymbol{\lambda} / \sqrt{3})$ values, namely (a) small to moderate $(0.01 \lesssim \boldsymbol{\lambda} \leq 0.1)$, (b) moderate to large $(0.1<\boldsymbol{\lambda} \leq 0.7)$ and (c) dominant (i.e., $\boldsymbol{\lambda}>0.7$ ).

In section 5 we have presented for the first-time a set of formulas for the singlet-like scalars, pseudoscalars and neutralinos mass terms (eq. (5.5)) assuming a simplified index structure (eq. (5.4)) of the relevant model parameters. We however, did not consider any assumption regarding the scale of the parameters. Hence, these formulae are rather generic and can also be applied for the NMSSM, with or without multiple singlets along with necessary changes (e.g., with one singlet $\mu=\lambda \nu^{c}$ etc.). We explored these mass terms for the same three different regions of $\lambda_{i}$ values. The scale of the other relevant parameters (e.g. $\kappa, \mu$, etc.) have also been estimated during our investigation.

Finally, in section 6 we have discussed the new two-body decays of $S_{4}^{0}$ into a pair of $S_{i}^{0}, P_{i}^{0}, \widetilde{\chi}_{i+3}^{0}$ states, presented the expressions of the decay widths (eq. (6.1)), and estimated the five well measured reduced signal strengths, namely $\mu_{\gamma \gamma}\left(S_{4}^{0}\right), \mu_{b \bar{b}}\left(S_{4}^{0}\right), \mu_{\tau^{+} \tau^{-}}\left(S_{4}^{0}\right)$, $\mu_{W W^{*}}\left(S_{4}^{0}\right)$ and $\mu_{Z Z^{*}}\left(S_{4}^{0}\right)$ (see eq. (6.15)), as well as the total decay width for the SM-like $S_{4}^{0}$ (eqs. (6.9) and (6.13)). Our discussion is furthermore extended to address the feasibility of getting prompt and/or displaced leptons/taus/jets/photons at colliders. These signatures appear through the decays of these new states, following the analyses presented in section 3, where issues of the possible leading backgrounds are also discussed. The final states considered in this article are the different combination of four prompt/displaced leptons/taus/jets/photons (at least two of the each type), accompanied by some $\not_{\mathrm{T}}$ originating from the light neutrinos $\left(\widetilde{\chi}_{i}^{0}\right)$ and/or possible mis-measurements. Our analysis also addressed the different possible natures (e.g., bino-like, right-handed neutrino-like or binosinglino mixed) of the lightest neutralino $\widetilde{\chi}_{4}^{0}$ and consequently the effect on the length of the associated displaced vertex.

In this course of analysis we have observed that in terms of the new $S_{4}^{0}$ decays, consistent with experimental observations, e.g. reduced signal strengths, the upper limit of total $S_{4}^{0}$ decay width, etc., small to moderate $\lambda_{i}$ values (i.e., $0.01 \lesssim \boldsymbol{\lambda} \leq 0.1$ ) are the most favoured one, given that one works in the range of $2.5 \lesssim \tan \beta \lesssim 3.9$. This range in $\tan \beta$ is estimated with our choice of $\left|R_{41}^{S^{0}}\right|^{2}=0.1$ and $\left|R_{42}^{S^{0}}\right|^{2}=0.9$. We have also shown the variation in the range of $\tan \beta$ with a different choice of $\left|R_{41}^{S^{0}}\right|^{2}$ and $\left|R_{42}^{S^{0}}\right|^{2}$, e.g. $1.45 \lesssim \tan \beta \lesssim 2.15$ with $\left|R_{42}^{S^{0}}\right|^{2}=0.75$ and $\left|R_{41}^{S^{0}}\right|^{2}=0.25$. Further, we have observed that the pure singlet-like $S_{i}^{0}, P_{i}^{0}$ and $\widetilde{\chi}_{i+3}^{0}$ states appear rather naturally in this corner of the parameter space with a negligible to small parameter tuning. This is also true for their lightness, as discussed in subsection 5.1. The room for a light $\widetilde{\chi}_{b}^{0}$, that can survive the collider constraints, exists for this region of the parameter space only when $\mu$ takes a large value. Note however that the advantage of getting relevant extra contribution to the tree-level lightest doublet-like scalar mass is hardly possible for this range of $\lambda_{i}$ values. An interesting observation for this corner of the parameter space is its capability of accommodating all the five reduced signal strengths $\approx 1$, the expected $\mathrm{SM}$ value, even in the presence of a non-vanishing new physics contribution.

For moderate to large $\lambda_{i}$ (i.e., $0.1<\boldsymbol{\lambda} \leq 0.7$ ), on the contrary, a sizable contribution to the tree-level lightest doublet-like scalar mass is possible for this region of the parameter 
space with small $\tan \beta$. With a larger singlet-doublet mixing (through larger $\lambda_{i}$ ), the singlet purity of $S_{i}^{0}, P_{i}^{0}$ and $\tilde{\chi}_{i+3}^{0}$ states diminishes for this corner of the parameter space. Their lightness can, however, be preserved through a fine cancellation using a moderate to large parameter tuning. Regarding decays, depending on the value of $\lambda_{i}, S_{4}^{0} \rightarrow S_{i}^{0} S_{j}^{0}$ and $S_{4}^{0} \rightarrow$ $P_{i}^{0} P_{j}^{0}$ processes can produce the experimentally unacceptable reduced signal strengths and the total decay width, while the displaced objects through $S_{4}^{0} \rightarrow \widetilde{\chi}_{i+3}^{0} \widetilde{\chi}_{j+3}^{0}$ modes still remain feasible. However, as $\boldsymbol{\lambda} \rightarrow 0.7$ the light $\widetilde{\chi}_{i+3}^{0}$ states must not be accompanied by lighter $S_{i}^{0}, P_{i}^{0}$ states in order to remain experimentally viable. A similar conclusion also holds true for a bino-like lightest neutralino for this entire region of $\boldsymbol{\lambda}$ values. The absence of lighter $S_{i}^{0}, P_{i}^{0}$ states leaves its imprint in terms of the associated decay length. For $\boldsymbol{\lambda}$ values closer to 0.2 , once again a small window of low $\tan \beta$ values, e.g. about $2.3 \lesssim \tan \beta \lesssim 3.75$, remains consistent with the experimental observations for the light singlino-like $\widetilde{\chi}_{i+3}^{0}$. For a light $\widetilde{\chi}_{b}^{0}$, on the other hand, depending on the scale of $\boldsymbol{\lambda}$, a similar range lies approximately within 1.6 to 3.85 . This range of $\tan \beta$ values, however, will change with a much different choice of $\left|R_{41}^{S^{0}}\right|^{2}$ and $\left|R_{42}^{S^{0}}\right|^{2}$.

Lastly, the dominant $\lambda_{i}$ region (i.e. $\boldsymbol{\lambda}>0.7$ ) mainly serves the purpose of giving a very large additional contribution to the tree-level lightest doublet-like scalar mass. The lightness of the $S_{i}^{0}, P_{i}^{0}$ and $\widetilde{\chi}_{i+3}^{0}$ states may still appear possible with a severe parameter tuning. With very large $\lambda_{i}$, these states appear with a very large singlet-doublet mixing and hence, are often excluded from the existing experimental results. Also in this region of the $\lambda_{i}$, new $S_{4}^{0}$ decay modes, primarily through $S_{i}^{0}, P_{i}^{0}$, are normally ruled out by the experimentally measured reduced signal strengths and the upper limit of the $S_{4}^{0}$ decay width. Regarding $\widetilde{\chi}_{i+3}^{0}$ states, the conclusion remain the same, especially concerning the measured reduced signal strengths. A light $\widetilde{\chi}_{b}^{0}$, on the other hand, remains experimentally viable in this region of the parameter space for a small window of low $\tan \beta$ values around $2.4 \lesssim \tan \beta \lesssim 3.8$, given the absence of any lighter $S_{i}^{0}, P_{i}^{0}$ states.

To probe the model origin of $S_{4}^{0}$, further investigation of these kinds of new Higgs decay modes with the dedicated experimental analyses (i.e., to detect soft, collimated and often displaced objects), would appear very relevant in the coming years, especially concerning the restart of the LHC in 2015 with the enhanced centre-of-mass energy and increased luminosity. Analyses of these kinds can exclude/narrow down the hitherto available nonstandard decay window for the observed SM-Higgs like scalar boson. With higher centreof-mass energy and enhanced luminosity, the possibilities of detecting these new decays and hence, indirect evidences of new physics beyond the SM (e.g., [51]), are also well envisaged. One would, however, require a full numerical estimate of these scenarios with a proper background analysis which we aim to cover with a set of forthcoming publications [183].

\section{Acknowledgments}

The work of PG and CM is supported in part by the Spanish MINECO under grant FPA2012-34694 and under 'Centro de Excelencia Severo Ochoa' Programme SEV-20120249, and by the Comunidad de Madrid under grant HEPHACOS S2009/ESP-1473. The work of DL is supported by the Argentinian CONICET. VAM acknowledges support by the 
Spanish MINECO under the project FPA2012-39055-C02-01, by the Generalitat Valenciana through the project PROMETEO II/2013-017 and by the Spanish National Research Council (CSIC) under the JAE-Doc program co-funded by the European Social Fund (ESF). The work of RR is supported by the Ramón y Cajal program of the Spanish MINECO and also thanks the support of the MINECO under grant FPA2011-29678. The authors also acknowledge the support of the MINECO's Consolider-Ingenio 2010 Programme under grant MultiDark CSD2009-00064.

Open Access. This article is distributed under the terms of the Creative Commons Attribution License (CC-BY 4.0), which permits any use, distribution and reproduction in any medium, provided the original author(s) and source are credited.

\section{References}

[1] ATLAS collaboration, Observation of a new particle in the search for the standard model Higgs boson with the ATLAS detector at the LHC, Phys. Lett. B 716 (2012) 1 [arXiv: 1207.7214] [INSPIRE].

[2] CMS collaboration, Observation of a new boson at a mass of $125 \mathrm{GeV}$ with the CMS experiment at the LHC, Phys. Lett. B $\mathbf{7 1 6}$ (2012) 30 [arXiv:1207.7235] [INSPIRE].

[3] ATLAS collaboration, A particle consistent with the Higgs boson observed with the ATLAS detector at the Large Hadron Collider, Science 338 (2012) 1576 [INSPIRE].

[4] CMS collaboration, Observation of a new boson with mass near $125 \mathrm{GeV}$ in pp collisions at $\sqrt{s}=7$ and $8 \mathrm{TeV}$, JHEP 06 (2013) 081 [arXiv:1303.4571] [INSPIRE].

[5] CMS collaboration, Evidence for the direct decay of the $125 \mathrm{GeV}$ Higgs boson to fermions, Nature Phys. 10 (2014) [arXiv:1401.6527] [inSPIRE].

[6] ATLAS collaboration, Measurements of Higgs boson production and couplings in diboson final states with the ATLAS detector at the LHC, Phys. Lett. B 726 (2013) 88 [arXiv: 1307.1427] [INSPIRE].

[7] ATLAS collaboration, Evidence for the spin-0 nature of the Higgs boson using ATLAS data, Phys. Lett. B 726 (2013) 120 [arXiv:1307.1432] [INSPIRE].

[8] CMS Collaboration, Combination of standard model Higgs boson searches and measurements of the properties of the new boson with a mass near $125 \mathrm{GeV}$, CMS-PAS-HIG-13-005 (2013).

[9] CMS collaboration, Search for a Higgs boson decaying into a $Z$ and a photon in pp collisions at $\sqrt{s}=7$ and 8 TeV, Phys. Lett. B 726 (2013) 587 [arXiv:1307.5515] [INSPIRE].

[10] CMS collaboration, Search for the standard model Higgs boson produced in association with a W or a $Z$ boson and decaying to bottom quarks, Phys. Rev. D 89 (2014) 012003 [arXiv:1310.3687] [INSPIRE].

[11] CMS collaboration, Measurement of Higgs boson production and properties in the $W W$ decay channel with leptonic final states, JHEP 01 (2014) 096 [arXiv:1312.1129] [INSPIRE].

[12] ATLAS collaboration, Updated coupling measurements of the Higgs boson with the ATLAS detector using up to $25 \mathrm{fb}^{-1}$ of proton-proton collision data, ATLAS-CONF-2014-009 (2014).

[13] CMS collaboration, Measurement of the properties of a Higgs boson in the four-lepton final state, Phys. Rev. D 89 (2014) 092007 [arXiv: 1312.5353] [INSPIRE]. 
[14] CMS collaboration, Evidence for the $125 \mathrm{GeV}$ Higgs boson decaying to a pair of $\tau$ leptons, JHEP 05 (2014) 104 [arXiv:1401.5041] [INSPIRE].

[15] ATLAS collaboration, Measurement of the Higgs boson mass from the $H \rightarrow \gamma \gamma$ and $H \rightarrow Z Z^{*} \rightarrow 4 \ell$ channels with the ATLAS detector using $25 \mathrm{fb}^{-1}$ of pp collision data, Phys. Rev. D 90 (2014) 052004 [arXiv:1406.3827] [INSPIRE].

[16] ATLAS collaboration, Search for the standard model Higgs boson decay to $\mu^{+} \mu^{-}$with the ATLAS detector, Phys. Lett. B 738 (2014) 68 [arXiv:1406.7663] [INSPIRE].

[17] CMS Collaboration, Precise determination of the mass of the Higgs boson and studies of the compatibility of its couplings with the standard model, CMS-PAS-HIG-14-009 (2014).

[18] CMS collaboration, Observation of the diphoton decay of the Higgs boson and measurement of its properties, Eur. Phys. J. C 74 (2014) 3076 [arXiv:1407.0558] [INSPIRE].

[19] ATLAS collaboration, Measurement of Higgs boson production in the diphoton decay channel in pp collisions at center-of-mass energies of 7 and 8 TeV with the ATLAS detector, arXiv:1408.7084 [INSPIRE].

[20] ATLAS SUSY searches - 95\% CL lower limits, see online.

[21] Summary of CMS SUSY results in SMS framework, see online.

[22] D.E. López-Fogliani and C. Muñoz, Proposal for a supersymmetric standard model, Phys. Rev. Lett. 97 (2006) 041801 [hep-ph/0508297] [INSPIRE].

[23] N. Escudero, D.E. López-Fogliani, C. Muñoz and R.R. de Austri, Analysis of the parameter space and spectrum of the mu nu SSM, JHEP 12 (2008) 099 [arXiv:0810.1507] [INSPIRE].

[24] J.E. Kim and H.P. Nilles, The mu Problem and the Strong CP Problem, Phys. Lett. B 138 (1984) 150 [InSPIRE].

[25] H.P. Nilles, Supersymmetry, supergravity and particle physics, Phys. Rept. 110 (1984) 1 [INSPIRE].

[26] H.E. Haber and G.L. Kane, The search for supersymmetry: probing physics beyond the standard model, Phys. Rept. 117 (1985) 75 [INSPIRE].

[27] I. Simonsen, A review of minimal supersymmetric electroweak theory, hep-ph/9506369 [INSPIRE].

[28] S.P. Martin, A supersymmetry primer, Adv. Ser. Direct. High Energy Phys. 21 (2010) 1 [hep-ph/9709356] [INSPIRE].

[29] P. Ghosh and S. Roy, Neutrino masses and mixing, lightest neutralino decays and a solution to the mu problem in supersymmetry, JHEP 04 (2009) 069 [arXiv:0812.0084] [INSPIRE].

[30] A. Bartl, M. Hirsch, A. Vicente, S. Liebler and W. Porod, LHC phenomenology of the $\mu \nu S S M$, JHEP 05 (2009) 120 [arXiv:0903.3596] [INSPIRE].

[31] J. Fidalgo, D.E. López-Fogliani, C. Muñoz and R. Ruiz de Austri, Neutrino physics and spontaneous CP-violation in the $\mu \nu S S M$, JHEP 08 (2009) 105 [arXiv:0904.3112] [INSPIRE].

[32] P. Ghosh, P. Dey, B. Mukhopadhyaya and S. Roy, Radiative contribution to neutrino masses and mixing in $\mu \nu S S M$, JHEP 05 (2010) 087 [arXiv:1002.2705] [INSPIRE].

[33] D.E. Lopez-Fogliani, The seesaw mechanism in the $\mu \nu S S M$, arXiv: 1004.0884 [INSPIRE].

[34] P. Ghosh, Neutrino masses and mixing in $\mu \nu S S M$, J. Phys. Conf. Ser. 259 (2010) 012063 [arXiv:1010.2578] [INSPIRE].

[35] D.V. Forero, M. Tortola and J.W.F. Valle, Global status of neutrino oscillation parameters after Neutrino-2012, Phys. Rev. D 86 (2012) 073012 [arXiv: 1205.4018] [INSPIRE]. 
[36] M.C. Gonzalez-Garcia, M. Maltoni, J. Salvado and T. Schwetz, Global fit to three neutrino mixing: critical look at present precision, JHEP 12 (2012) 123 [arXiv:1209.3023] [INSPIRE].

[37] F. Capozzi et al., Status of three-neutrino oscillation parameters, circa 2013, Phys. Rev. D 89 (2014) 093018 [arXiv:1312.2878] [INSPIRE].

[38] P. Fayet, Supergauge invariant extension of the Higgs mechanism and a model for the electron and its neutrino, Nucl. Phys. B 90 (1975) 104 [INSPIRE].

[39] P. Fayet, Spontaneously broken supersymmetric theories of weak, electromagnetic and strong interactions, Phys. Lett. B 69 (1977) 489 [INSPIRE].

[40] G.R. Farrar and P. Fayet, Phenomenology of the production, decay and detection of new hadronic states associated with supersymmetry, Phys. Lett. B 76 (1978) 575 [INSPIRE].

[41] S. Weinberg, Supersymmetry at ordinary energies. 1. Masses and conservation laws, Phys. Rev. D 26 (1982) 287 [INSPIRE].

[42] C.S. Aulakh and R.N. Mohapatra, Neutrino as the supersymmetric partner of the Majoron, Phys. Lett. B 119 (1982) 136 [INSPIRE].

[43] L.J. Hall and M. Suzuki, Explicit R-parity breaking in supersymmetric models, Nucl. Phys. B 231 (1984) 419 [InSPIRE].

[44] I.-H. Lee, Lepton number violation in softly broken supersymmetry, Phys. Lett. B 138 (1984) 121 [INSPIRE].

[45] I.-H. Lee, Lepton number violation in softly broken supersymmetry. 2, Nucl. Phys. B 246 (1984) 120 [InSPIRE].

[46] G.G. Ross and J.W.F. Valle, Supersymmetric models without R-parity, Phys. Lett. B 151 (1985) 375 [INSPIRE].

[47] J.R. Ellis, G. Gelmini, C. Jarlskog, G.G. Ross and J.W.F. Valle, Phenomenology of supersymmetry with broken R-parity, Phys. Lett. B 150 (1985) 142 [InSPIRE].

[48] P. Bandyopadhyay, P. Ghosh and S. Roy, Unusual Higgs boson signal in R-parity violating nonminimal supersymmetric models at the LHC, Phys. Rev. D 84 (2011) 115022 [arXiv: 1012.5762] [INSPIRE].

[49] J. Fidalgo, D.E. Lopez-Fogliani, C. Muñoz and R.R. de Austri, The Higgs sector of the $\mu \nu$ SSM and collider physics, JHEP 10 (2011) 020 [arXiv:1107.4614] [INSPIRE].

[50] P. Ghosh, D.E. Lopez-Fogliani, V.A. Mitsou, C. Muñoz and R. Ruiz de Austri, Probing the $\mu$-from- $\nu$ supersymmetric standard model with displaced multileptons from the decay of a Higgs boson at the LHC, Phys. Rev. D 88 (2013) 015009 [arXiv:1211.3177] [INSPIRE].

[51] P. Ghosh, D.E. Lopez-Fogliani, V.A. Mitsou, C. Muñoz and R. Ruiz de Austri, Hunting physics beyond the standard model with unusual $W^{ \pm}$and $Z$ decays, arXiv:1403.3675 [INSPIRE].

[52] J. Erler and S. Heinemeyer, GigaZ: high precision tests of the SM and the MSSM, hep-ph/0102083 [INSPIRE].

[53] American Linear Collider Working Group collaboration, T. Abe et al., Linear collider physics resource book for Snowmass 2001 - Part 2: Higgs and supersymmetry studies, hep-ex/0106056 [INSPIRE].

[54] American Linear Collider Working Group collaboration, T. Abe et al., Linear collider physics resource book for Snowmass 2001 - Part 3: studies of exotic and standard model physics, hep-ex/0106057 [INSPIRE]. 
[55] S. Heinemeyer and G. Weiglein, Top, GigaZ, MegaW, arXiv:1007.5232 [INSPIRE].

[56] TleP Design Study Working Group collaboration, M. Bicer et al., First look at the physics case of TLEP, JHEP 01 (2014) 164 [arXiv: 1308.6176] [INSPIRE].

[57] K.-Y. Choi, D.E. Lopez-Fogliani, C. Muñoz and R.R. de Austri, Gamma-ray detection from gravitino dark matter decay in the $\mu \nu S S M$, JCAP 03 (2010) 028 [arXiv:0906.3681] [INSPIRE].

[58] G.A. Gomez-Vargas et al., CLUES on Fermi-LAT prospects for the extragalactic detection of $\mu \nu$ SSM gravitino Dark Matter, JCAP 02 (2012) 001 [arXiv:1110.3305] [INSPIRE].

[59] C. Muñoz, Searches for gamma-ray lines from $\mu \nu S S M$ gravitino dark matter, PoS (DSU 2012) 045.

[60] Fermi-LAT collaboration, A. Albert et al., Search for $100 \mathrm{MeV}$ to $10 \mathrm{GeV} \gamma$-ray lines in the Fermi-LAT data and implications for gravitino dark matter in $\mu \nu S S M$, JCAP 10 (2014) 023 [arXiv:1406.3430] [InSPIRE].

[61] D.J.H. Chung and A.J. Long, Electroweak phase transition in the $\mu \nu S S M$, Phys. Rev. D 81 (2010) 123531 [arXiv:1004.0942] [INSPIRE].

[62] J.F. Gunion, H.E. Haber and T. Moroi, Will at least one of the Higgs bosons of the next-to-minimal supersymmetric extension of the standard model be observable at LEP-2 or the LHC?, eConf C 960625 (1996) LTH095 [hep-ph/9610337] [INSPIRE].

[63] U. Ellwanger, J.F. Gunion and C. Hugonie, Establishing a no lose theorem for NMSSM Higgs boson discovery at the LHC, hep-ph/0111179 [INSPIRE].

[64] U. Ellwanger, J.F. Gunion, C. Hugonie and S. Moretti, Towards a no lose theorem for NMSSM Higgs discovery at the LHC, hep-ph/0305109 [INSPIRE].

[65] U. Ellwanger, J.F. Gunion, C. Hugonie and S. Moretti, NMSSM Higgs discovery at the LHC, hep-ph/0401228 [INSPIRE].

[66] R. Dermisek and J.F. Gunion, Escaping the large fine tuning and little hierarchy problems in the next to minimal supersymmetric model and $h \rightarrow$ aa decays, Phys. Rev. Lett. 95 (2005) 041801 [hep-ph/0502105] [INSPIRE].

[67] R. Dermisek and J.F. Gunion, Consistency of LEP event excesses with an $h \rightarrow$ aa decay scenario and low-fine-tuning NMSSM models, Phys. Rev. D 73 (2006) 111701 [hep-ph/0510322] [INSPIRE].

[68] P.C. Schuster and N. Toro, Persistent fine-tuning in supersymmetry and the NMSSM, hep-ph/0512189 [inSPIRE].

[69] R. Dermisek and J.F. Gunion, The NMSSM close to the R-symmetry limit and naturalness in $h \rightarrow$ aa decays for $m_{a}<2 m_{b}$, Phys. Rev. D 75 (2007) 075019 [hep-ph/0611142] [INSPIRE].

[70] S.-h. Zhu, Unique Higgs boson signature at colliders, hep-ph/0611270 [INSPIRE].

[71] R. Dermisek and J.F. Gunion, The NMSSM solution to the fine-tuning problem, precision electroweak constraints and the largest LEP Higgs event excess,

Phys. Rev. D 76 (2007) 095006 [arXiv:0705.4387] [InSPIRE].

[72] M. Carena, T. Han, G.-Y. Huang and C.E.M. Wagner, Higgs signal for $h \rightarrow$ aa at hadron colliders, JHEP 04 (2008) 092 [arXiv: 0712.2466] [INSPIRE].

[73] A. Belyaev et al., The scope of the $4 \tau$ channel in Higgs-strahlung and vector boson fusion for the NMSSM no-lose theorem at the LHC, arXiv:0805.3505 [INSPIRE]. 
[74] R. Dermisek and J.F. Gunion, Many light Higgs bosons in the NMSSM, Phys. Rev. D 79 (2009) 055014 [arXiv:0811.3537] [inSPIRE].

[75] M. Lisanti and J.G. Wacker, Discovering the Higgs with low mass muon pairs, Phys. Rev. D 79 (2009) 115006 [arXiv:0903.1377] [inSPIRE].

[76] A. Belyaev, J. Pivarski, A. Safonov, S. Senkin and A. Tatarinov, LHC discovery potential of the lightest NMSSM Higgs in the $H_{1} \rightarrow a_{1} a_{1} \rightarrow 4$ muons channel, Phys. Rev. D 81 (2010) 075021 [arXiv:1002.1956] [inSPIRE].

[77] M.M. Almarashi and S. Moretti, Low mass Higgs signals at the LHC in the next-to-minimal supersymmetric standard model, Eur. Phys. J. C 71 (2011) 1618 [arXiv:1011.6547] [INSPIRE].

[78] M.M. Almarashi and S. Moretti, Muon signals of very light CP-odd Higgs states of the NMSSM at the LHC, Phys. Rev. D 83 (2011) 035023 [arXiv:1101.1137] [InSPIRE].

[79] M. Almarashi and S. Moretti, Very light CP-odd Higgs bosons of the NMSSM at the LHC in 4b-quark final states, Phys. Rev. D 84 (2011) 015014 [arXiv:1105.4191] [InSPIRE].

[80] U. Ellwanger, Higgs bosons in the next-to-minimal supersymmetric standard model at the LHC, Eur. Phys. J. C 71 (2011) 1782 [arXiv:1108.0157] [inSPIRE].

[81] M.M. Almarashi and S. Moretti, LHC signals of a heavy CP-even Higgs boson in the NMSSM via decays into a $Z$ and a light CP-odd Higgs state, Phys. Rev. D 85 (2012) 017701 [arXiv:1109.1735] [INSPIRE].

[82] J. Rathsman and T. Rossler, Closing the window on light charged Higgs bosons in the NMSSM, Adv. High Energy Phys. 2012 (2012) 853706 [arXiv:1206.1470] [INSPIRE].

[83] R. Dermisek, E. Lunghi and A. Raval, Trilepton signatures of light charged and CP-odd Higgs bosons in top quark decays, JHEP 04 (2013) 063 [arXiv:1212.5021] [INSPIRE].

[84] Z. Kang, J. Li, T. Li, D. Liu and J. Shu, Probing the CP-even Higgs sector via $\mathrm{H}_{3} \rightarrow \mathrm{H}_{2} \mathrm{H}_{1}$ in the natural next-to-minimal supersymmetric standard model,

Phys. Rev. D 88 (2013) 015006 [arXiv:1301.0453] [inSPIRE].

[85] D.G. Cerdeno, P. Ghosh and C.B. Park, Probing the two light Higgs scenario in the NMSSM with a low-mass pseudoscalar, JHEP 06 (2013) 031 [arXiv:1301.1325] [INSPIRE].

[86] B. Bhattacherjee et al., Implications of the $98 \mathrm{GeV}$ and $125 \mathrm{GeV}$ Higgs scenarios in nondecoupling supersymmetry with updated ATLAS, CMS and PLANCK data, Phys. Rev. D 88 (2013) 035011 [arXiv: 1305.4020] [INSPIRE].

[87] D.G. Cerdeño, P. Ghosh, C.B. Park and M. Peiró, Collider signatures of a light NMSSM pseudoscalar in neutralino decays in the light of LHC results, JHEP 02 (2014) 048 [arXiv: 1307.7601] [INSPIRE].

[88] J. Cao, F. Ding, C. Han, J.M. Yang and J. Zhu, A light Higgs scalar in the NMSSM confronted with the latest LHC Higgs data, JHEP 11 (2013) 018 [arXiv:1309.4939] [INSPIRE].

[89] R. Dermisek, J.P. Hall, E. Lunghi and S. Shin, A new avenue to charged Higgs discovery in multi-Higgs models, JHEP 04 (2014) 140 [arXiv:1311.7208] [INSPIRE].

[90] B. Grinstein, C.W. Murphy, D. Pirtskhalava and P. Uttayarat, Theoretical constraints on additional Higgs bosons in light of the $126 \mathrm{GeV}$ Higgs, JHEP 05 (2014) 083 [arXiv:1401.0070] [INSPIRE].

[91] U. Ellwanger and A.M. Teixeira, NMSSM with a singlino LSP: possible challenges for searches for supersymmetry at the LHC, JHEP 10 (2014) 113 [arXiv:1406.7221] [INSPIRE]. 
[92] S.F. King, M. Muhlleitner, R. Nevzorov and K. Walz, Discovery prospects for NMSSM Higgs bosons at the high-energy Large Hadron Collider, arXiv:1408.1120 [INSPIRE].

[93] D. Das, L. Mitzka and W. Porod, Discovery of charged Higgs through $\gamma \gamma$ final states, arXiv: 1408.1704 [INSPIRE].

[94] N.-E. Bomark, S. Moretti, S. Munir and L. Roszkowski, A light NMSSM pseudoscalar Higgs boson at the LHC redux, arXiv: 1409.8393 [INSPIRE].

[95] OPAL collaboration, G. Abbiendi et al., Decay mode independent searches for new scalar bosons with the OPAL detector at LEP, Eur. Phys. J. C 27 (2003) 311 [hep-ex/0206022] [INSPIRE].

[96] OPAL collaboration, G. Abbiendi et al., Search for a low mass CP odd Higgs boson in $e^{+} e^{-}$collisions with the OPAL detector at LEP-2, Eur. Phys. J. C 27 (2003) 483 [hep-ex/0209068] [INSPIRE].

[97] LeP Working Group for Higgs boson searches, AlEPH, DELPHi, L3, OPAL collaboration, R. Barate et al., Search for the standard model Higgs boson at LEP, Phys. Lett. B 565 (2003) 61 [hep-ex/0306033] [INSPIRE].

[98] OPAL collaboration, G. Abbiendi et al., Search for neutral Higgs boson in CP-conserving and CP-violating MSSM scenarios, Eur. Phys. J. C 37 (2004) 49 [hep-ex/0406057] [INSPIRE].

[99] DELPHI collaboration, J. Abdallah et al., Searches for neutral Higgs bosons in extended models, Eur. Phys. J. C 38 (2004) 1 [hep-ex/0410017] [InSPIRE].

[100] ALEPH, DELPhi, L3, OPAL, LEP Working Group for Higgs Boson Searches collaboration, S. Schael et al., Search for neutral MSSM Higgs bosons at LEP, Eur. Phys. J. C 47 (2006) 547 [hep-ex/0602042] [INSPIRE].

[101] ALEPH collaboration, J. Beacham, Higgs to four taus at ALEPH, arXiv:1006.2491 [INSPIRE].

[102] D0 collaboration, V.M. Abazov et al., Search for NMSSM Higgs bosons in the $h \rightarrow a a \rightarrow \mu \mu \mu \mu, \mu \mu \tau \tau$ channels using pp collisions at $\sqrt{s}=1.96 \mathrm{TeV}$, Phys. Rev. Lett. 103 (2009) 061801 [arXiv:0905.3381] [INSPIRE].

[103] M. Klemetti, Search for Higgs bosons beyond the Standard Model in early ATLAS data, CERN-THESIS-2011-024 (2011).

[104] ATLAS collaboration, A search for light CP-odd Higgs bosons decaying to $\mu^{+} \mu^{-}$in ATLAS, ATLAS-CONF-2011-020 (2011).

[105] ATLAS collaboration, Search for a Higgs boson decaying to four photons through light CP-odd scalar coupling using $4.9 \mathrm{fb} \mathrm{b}^{1-}$ of $7 \mathrm{TeV}$ pp collision data taken with ATLAS detector at the LHC, ATLAS-CONF-2012-079 (2012).

[106] ATLAS collaboration, Search for the standard model Higgs boson in $H \rightarrow \tau \tau$ decays in proton-proton collisions with the ATLAS detector, ATLAS-CONF-2012-160 (2012).

[107] CMS collaboration, Search for a non-standard-model Higgs boson decaying to a pair of new light bosons in four-muon final states, Phys. Lett. B 726 (2013) 564 [arXiv:1210.7619] [INSPIRE].

[108] CMS collaboration, Search for a non-standard-model Higgs boson decaying to a pair of new light bosons in four-muon final states, CMS-PAS-HIG-13-010 (2013).

[109] CLEO collaboration, W. Love et al., Search for very light CP-odd Higgs boson in radiative decays of $\Upsilon(S-1)$, Phys. Rev. Lett. 101 (2008) 151802 [arXiv:0807.1427] [InSPIRE]. 
[110] F. Domingo, U. Ellwanger, E. Fullana, C. Hugonie and M.-A. Sanchis-Lozano, Radiative $\Upsilon$ decays and a light pseudoscalar Higgs in the NMSSM, JHEP 01 (2009) 061 [arXiv:0810.4736] [INSPIRE].

[111] BABAR collaboration, B. Aubert et al., Search for dimuon decays of a light scalar boson in radiative transitions $\Upsilon \rightarrow \gamma A_{0}$, Phys. Rev. Lett. 103 (2009) 081803 [arXiv:0905.4539] [INSPIRE].

[112] BaBAr collaboration, B. Aubert et al., Search for a low-mass Higgs boson in $\Upsilon(3 S) \rightarrow \gamma A_{0}$, $A_{0} \rightarrow \tau^{+} \tau^{-}$at BABAR, Phys. Rev. Lett. 103 (2009) 181801 [arXiv:0906.2219] [INSPIRE].

[113] R. Dermisek and J.F. Gunion, New constraints on a light CP-odd Higgs boson and related NMSSM ideal Higgs scenarios, Phys. Rev. D 81 (2010) 075003 [arXiv:1002.1971] [INSPIRE].

[114] S. Andreas, O. Lebedev, S. Ramos-Sanchez and A. Ringwald, Constraints on a very light CP-odd Higgs of the NMSSM and other axion-like particles, JHEP 08 (2010) 003 [arXiv: 1005.3978] [INSPIRE].

[115] BABAR collaboration, P. del Amo Sanchez et al., Search for production of invisible final states in single-photon decays of $\Upsilon(1 S)$, Phys. Rev. Lett. 107 (2011) 021804 [arXiv: 1007.4646] [INSPIRE].

[116] BABAR collaboration, J.P. Lees et al., Search for hadronic decays of a light Higgs boson in the radiative decay $\Upsilon \rightarrow \gamma A^{0}$, Phys. Rev. Lett. 107 (2011) 221803 [arXiv:1108.3549] [INSPIRE].

[117] BESIII collaboration, M. Ablikim et al., Search for a light Higgs-like boson $A^{0}$ in $J / \psi$ radiative decays, Phys. Rev. D 85 (2012) 092012 [arXiv:1111.2112] [INSPIRE].

[118] BABAR collaboration, J.P. Lees et al., Search for di-muon decays of a low-mass Higgs boson in radiative decays of the $\Upsilon(1 S)$, Phys. Rev. D 87 (2013) 031102 [arXiv:1210.0287] [INSPIRE].

[119] BABAR collaboration, J.P. Lees et al., Search for a low-mass scalar Higgs boson decaying to a $\tau$ pair in single-photon decays of $\Upsilon(1 S)$, Phys. Rev. D 88 (2013) 071102 [arXiv:1210.5669] [INSPIRE].

[120] BABAR collaboration, J.P. Lees et al., Search for a light Higgs boson decaying to two gluons or $s \bar{s}$ in the radiative decays of $\Upsilon(1 S)$, Phys. Rev. D 88 (2013) 031701 [arXiv:1307.5306] [INSPIRE].

[121] E. Accomando et al., Workshop on CP studies and non-standard Higgs physics, hep-ph/0608079 [INSPIRE].

[122] P. Draper, T. Liu, C.E.M. Wagner, L.-T. Wang and H. Zhang, Dark light Higgs, Phys. Rev. Lett. 106 (2011) 121805 [arXiv: 1009.3963] [InSPIRE].

[123] J. Huang, T. Liu, L.-T. Wang and F. Yu, Supersymmetric exotic decays of the $125 \mathrm{GeV}$ Higgs boson, Phys. Rev. Lett. 112 (2014) 221803 [arXiv: 1309.6633] [INSPIRE].

[124] D. Curtin et al., Exotic decays of the 125 GeV Higgs boson, Phys. Rev. D 90 (2014) 075004 [arXiv: 1312.4992] [INSPIRE].

[125] J. Huang, T. Liu, L.-T. Wang and F. Yu, Supersymmetric sub-electroweak scale dark matter, the galactic center gamma-ray excess and exotic decays of the $125 \mathrm{GeV}$ Higgs boson, arXiv: 1407.0038 [INSPIRE].

[126] H. Goldberg, Constraint on the photino mass from cosmology, Phys. Rev. Lett. 50 (1983) 1419 [Erratum ibid. 103 (2009) 099905] [InSPIRE]. 
[127] J.R. Ellis, J.S. Hagelin, D.V. Nanopoulos, K.A. Olive and M. Srednicki, Supersymmetric relics from the Big Bang, Nucl. Phys. B 238 (1984) 453 [INSPIRE].

[128] CMS collaboration, Constraints on the Higgs boson width from off-shell production and decay to Z-boson pairs, Phys. Lett. B 736 (2014) 64 [arXiv:1405.3455] [INSPIRE].

[129] ATLAS collaboration, Determination of the off-shell Higgs boson signal strength in the high-mass ZZ final state with the ATLAS detector, ATLAS-CONF-2014-042 (2014).

[130] CMS Collaboration, Constraints on the Higgs boson width from off-shell production and decay $\rightarrow Z Z \rightarrow$ llll and ll $\nu \nu$, CMS-PAS-HIG-14-002 (2014).

[131] ATLAS collaboration, Search for the bb decay of the standard model Higgs boson in associated W/ZH production with the ATLAS detector, ATLAS-CONF-2013-079 (2013).

[132] CMS collaboration, Search for Higgs boson production in association with a top-quark pair and decaying to bottom quarks or tau leptons, CMS-PAS-HIG-13-019 (2013).

[133] CMS collaboration, Higgs to $\tau \tau$ (MSSM), CMS-PAS-HIG-13-021 (2013).

[134] N. Desai, B. Mukhopadhyaya and S. Niyogi, Constraints on invisible Higgs decay in MSSM in the light of diphoton rates from the LHC, arXiv:1202.5190 [INSPIRE].

[135] J.R. Espinosa, M. Muhlleitner, C. Grojean and M. Trott, Probing for invisible Higgs decays with global fits, JHEP 09 (2012) 126 [arXiv:1205.6790] [INSPIRE].

[136] D. Ghosh, R. Godbole, M. Guchait, K. Mohan and D. Sengupta, Looking for an invisible Higgs signal at the LHC, Phys. Lett. B 725 (2013) 344 [arXiv:1211.7015] [INSPIRE].

[137] ATLAS collaboration, Search for invisible decays of a Higgs boson produced in association with a $Z$ boson in ATLAS, ATLAS-CONF-2013-011 (2013).

[138] CMS Collaboration, Search for an invisible Higgs boson, CMS-PAS-HIG-13-013 (2013).

[139] CMS Collaboration, Search for invisible Higgs produced in association with a $Z$ boson, CMS-PAS-HIG-13-018 (2013).

[140] CMS Collaboration, Search for the Higgs boson decaying to invisible particles produced in association with $Z$ bosons decaying to bottom quarks, CMS-PAS-HIG-13-028 (2013).

[141] G. Bélanger, B. Dumont, U. Ellwanger, J.F. Gunion and S. Kraml, Status of invisible Higgs decays, Phys. Lett. B 723 (2013) 340 [arXiv:1302.5694] [INSPIRE].

[142] J. Ellis and T. You, Updated global analysis of Higgs couplings, JHEP 06 (2013) 103 [arXiv:1303.3879] [INSPIRE].

[143] B. Ananthanarayan, J. Lahiri, P.N. Pandita and M. Patra, Invisible decays of the lightest Higgs boson in supersymmetric models, Phys. Rev. D 87 (2013) 115021 [arXiv:1306.1291] [INSPIRE].

[144] G. Bélanger, B. Dumont, U. Ellwanger, J.F. Gunion and S. Kraml, Global fit to Higgs signal strengths and couplings and implications for extended Higgs sectors, Phys. Rev. D 88 (2013) 075008 [arXiv:1306.2941] [INSPIRE].

[145] ATLAS collaboration, Search for dark matter in events with a hadronically decaying $W$ or $Z$ boson and missing transverse momentum in pp collisions at $\sqrt{s}=8 \mathrm{TeV}$ with the ATLAS detector, Phys. Rev. Lett. 112 (2014) 041802 [arXiv:1309.4017] [INSPIRE].

[146] ATLAS collaboration, Search for invisible decays of a Higgs boson produced in association with a Z boson in ATLAS, Phys. Rev. Lett. 112 (2014) 201802 [arXiv:1402.3244] [INSPIRE]. 
[147] CMS collaboration, Search for invisible decays of Higgs bosons in the vector boson fusion and associated ZH production modes, Eur. Phys. J. C 74 (2014) 2980 [arXiv:1404.1344] [INSPIRE].

[148] P.N. Pandita and M. Patra, Invisible decays of low mass Higgs bosons in supersymmetric models, Phys. Rev. D 89 (2014) 115010 [arXiv:1405.7163] [INSPIRE].

[149] U. Ellwanger, C. Hugonie and A.M. Teixeira, The next-to-minimal supersymmetric standard model, Phys. Rept. 496 (2010) 1 [arXiv:0910.1785] [InSPIRE].

[150] R. Barbier et al., R-parity violating supersymmetry, Phys. Rept. 420 (2005) 1 [hep-ph/0406039] [INSPIRE].

[151] C. Bobeth, T. Ewerth, F. Krüger and J. Urban, Analysis of neutral Higgs boson contributions to the decays $\bar{B}_{s} \rightarrow \ell^{+} \ell^{-}$and $\bar{B} \rightarrow K \ell^{+} \ell^{-}$, Phys. Rev. D 64 (2001) 074014 [hep-ph/0104284] [INSPIRE].

[152] R.L. Arnowitt, B. Dutta, T. Kamon and M. Tanaka, Detection of $B_{s} \rightarrow \mu^{+} \mu^{-}$at the Tevatron run II and constraints on the SUSY parameter space, Phys. Lett. B 538 (2002) 121 [hep-ph/0203069] [INSPIRE].

[153] A.G. Akeroyd, F. Mahmoudi and D.M. Santos, The decay $B_{s} \rightarrow \mu^{+} \mu^{-}$: updated SUSY constraints and prospects, JHEP 12 (2011) 088 [arXiv:1108.3018] [INSPIRE].

[154] A. Arbey, M. Battaglia and F. Mahmoudi, Constraints on the MSSM from the Higgs sector: a pMSSM study of Higgs searches, $B_{s}^{0} \rightarrow \mu^{+} \mu^{-}$and dark matter direct detection, Eur. Phys. J. C 72 (2012) 1906 [arXiv:1112.3032] [INSPIRE].

[155] J. Cao, Z. Heng, D. Li and J.M. Yang, Current experimental constraints on the lightest Higgs boson mass in the constrained MSSM, Phys. Lett. B 710 (2012) 665 [arXiv: 1112.4391] [INSPIRE].

[156] F. Mahmoudi, S. Neshatpour and J. Orloff, Supersymmetric constraints from $B_{s} \rightarrow \mu^{+} \mu^{-}$ and $B \rightarrow K * \mu^{+} \mu^{-}$observables, JHEP 08 (2012) 092 [arXiv:1205.1845] [INSPIRE].

[157] A. Arbey, M. Battaglia, A. Djouadi and F. Mahmoudi, The Higgs sector of the phenomenological MSSM in the light of the Higgs boson discovery, JHEP 09 (2012) 107 [arXiv: 1207.1348] [INSPIRE].

[158] W. Altmannshofer, M. Carena, N.R. Shah and F. Yu, Indirect probes of the MSSM after the Higgs discovery, JHEP 01 (2013) 160 [arXiv:1211.1976] [INSPIRE].

[159] A. Arbey, M. Battaglia, F. Mahmoudi and D. Martinez Santos, Supersymmetry confronts $B_{s} \rightarrow \mu^{+} \mu^{-}$: present and future status, Phys. Rev. D 87 (2013) 035026 [arXiv:1212.4887] [INSPIRE].

[160] A. Arbey, M. Battaglia and F. Mahmoudi, Supersymmetric heavy Higgs bosons at the LHC, Phys. Rev. D 88 (2013) 015007 [arXiv:1303.7450] [InSPIRE].

[161] ATLAS collaboration, Search for the neutral Higgs bosons of the minimal supersymmetric standard model in pp collisions at $\sqrt{s}=7$ TeV with the ATLAS detector, JHEP 02 (2013) 095 [arXiv: 1211.6956] [INSPIRE].

[162] CMS collaboration, Search for neutral MSSM Higgs bosons decaying to a pair of tau leptons in pp collisions, JHEP 10 (2014) 160 [arXiv:1408.3316] [INSPIRE].

[163] CMS collabroation, CMS physics: technical design report volume 1: detector performance and software, CERN-LHCC-2006-001 (2006).

[164] ATLAS collaboration, Performance of the ATLAS $\tau$ trigger in 2011, ATLAS-CONF-2013-006 (2013). 
[165] ATLAS collaboration, J. Mahlstedt, The ATLAS hadronic tau trigger, J. Phys. Conf. Ser. 513 (2014) 012021 [inSPIRE].

[166] ATLAS collaboration, Calibration of b-tagging using dileptonic top pair events in a combinatorial likelihood approach with the ATLAS experiment, ATLAS-CONF-2014-004 (2014).

[167] B.A. Dobrescu, G.L. Landsberg and K.T. Matchev, Higgs boson decays to CP odd scalars at the Tevatron and beyond, Phys. Rev. D 63 (2001) 075003 [hep-ph/0005308] [INSPIRE].

[168] S. Chang, P.J. Fox and N. Weiner, Visible cascade Higgs decays to four photons at hadron colliders, Phys. Rev. Lett. 98 (2007) 111802 [hep-ph/0608310] [INSPIRE].

[169] C.G. Lester and D.J. Summers, Measuring masses of semiinvisibly decaying particles pair produced at hadron colliders, Phys. Lett. B 463 (1999) 99 [hep-ph/9906349] [INSPIRE].

[170] A. Barr, C. Lester and P. Stephens, $m(T 2)$ : the truth behind the glamour, J. Phys. G 29 (2003) 2343 [hep-ph/0304226] [INSPIRE].

[171] R. Kitano and K.-y. Oda, Neutrino masses in the supersymmetric standard model with right-handed neutrinos and spontaneous R-parity violation, Phys. Rev. D 61 (2000) 113001 [hep-ph/9911327] [INSPIRE].

[172] Particle Data Group collaboration, J. Beringer et al., Review of particle physics, Phys. Rev. D 86 (2012) 010001 [inSPIRE].

[173] S. Heinemeyer, W. Hollik, A.M. Weber and G. Weiglein, Z pole observables in the MSSM, JHEP 04 (2008) 039 [arXiv:0710.2972] [InSPIRE].

[174] H.K. Dreiner et al., Mass bounds on a very light neutralino, Eur. Phys. J. C 62 (2009) 547 [arXiv:0901.3485] [INSPIRE].

[175] R. Adhikari and B. Mukhopadhyaya, Light neutralinos in B decays, Phys. Rev. D 52 (1995) 3125 [hep-ph/9411347] [INSPIRE].

[176] H.K. Dreiner et al., Rare meson decays into very light neutralinos, Phys. Rev. D 80 (2009) 035018 [arXiv:0905.2051] [INSPIRE].

[177] H.K. Dreiner, J.S. Kim and O. Lebedev, First LHC constraints on neutralinos, Phys. Lett. B $\mathbf{7 1 5}$ (2012) 199 [arXiv:1206.3096] [INSPIRE].

[178] S. Dawson, R-parity breaking in supersymmetric theories, Nucl. Phys. B 261 (1985) 297 [INSPIRE].

[179] R. Hempfling, SUSY without R-parity: symmetry breaking and LSP phenomenology, hep-ph/9702412 [inSPIRE].

[180] B. Mukhopadhyaya and S. Roy, Radiative decay of the lightest neutralino in an R-parity violating supersymmetric theory, Phys. Rev. D 60 (1999) 115012 [hep-ph/9903418] [INSPIRE].

[181] U. Ellwanger and C. Hugonie, Neutralino cascades in the $(M+1) S S M$, Eur. Phys. J. C 5 (1998) 723 [hep-ph/9712300] [INSPIRE].

[182] U. Ellwanger and C. Hugonie, Topologies of the (M+1)SSM with a singlino LSP at LEP-2, Eur. Phys. J. C 13 (2000) 681 [hep-ph/9812427] [INSPIRE].

[183] P. Ghosh, D.E. López-Fogliani, V.A. Mitsou, C. Muñoz, and R.R. de Austri, Searching for new physics at the LHC with a $125 \mathrm{GeV}$ Higgs with the $\mu \nu S S M$, in preparation.

[184] M. Drees, Supersymmetric models with extended Higgs sector, Int. J. Mod. Phys. A 4 (1989) 3635 [inSPIRE]. 
[185] J.R. Ellis, J.F. Gunion, H.E. Haber, L. Roszkowski and F. Zwirner, Higgs bosons in a nonminimal supersymmetric model, Phys. Rev. D 39 (1989) 844 [InSPIRE].

[186] P. Binetruy and C.A. Savoy, Higgs and top masses in a nonminimal supersymmetric theory, Phys. Lett. B 277 (1992) 453 [INSPIRE].

[187] J.R. Espinosa and M. Quirós, On Higgs boson masses in nonminimal supersymmetric standard models, Phys. Lett. B 279 (1992) 92 [INSPIRE].

[188] J.R. Espinosa and M. Quirós, Upper bounds on the lightest Higgs boson mass in general supersymmetric standard models, Phys. Lett. B 302 (1993) 51 [hep-ph/9212305] [INSPIRE].

[189] H.E. Haber and R. Hempfling, Can the mass of the lightest Higgs boson of the minimal supersymmetric model be larger than $m(Z)$ ?, Phys. Rev. Lett. 66 (1991) 1815 [INSPIRE].

[190] G. Altarelli and R. Barbieri, Vacuum polarization effects of new physics on electroweak processes, Phys. Lett. B 253 (1991) 161 [INSPIRE].

[191] R. Hempfling and A.H. Hoang, Two loop radiative corrections to the upper limit of the lightest Higgs boson mass in the minimal supersymmetric model, Phys. Lett. B 331 (1994) 99 [hep-ph/9401219] [INSPIRE].

[192] J.A. Casas, J.R. Espinosa, M. Quirós and A. Riotto, The lightest Higgs boson mass in the minimal supersymmetric standard model, Nucl. Phys. B 436 (1995) 3 [Erratum ibid. B 439 (1995) 466] [hep-ph/9407389] [INSPIRE].

[193] M.S. Carena, J.R. Espinosa, M. Quirós and C.E.M. Wagner, Analytical expressions for radiatively corrected Higgs masses and couplings in the MSSM, Phys. Lett. B 355 (1995) 209 [hep-ph/9504316] [InSPIRE].

[194] M.S. Carena, M. Quirós and C.E.M. Wagner, Effective potential methods and the Higgs mass spectrum in the MSSM, Nucl. Phys. B 461 (1996) 407 [hep-ph/9508343] [INSPIRE].

[195] H.E. Haber, R. Hempfling and A.H. Hoang, Approximating the radiatively corrected Higgs mass in the minimal supersymmetric model, Z. Phys. C 75 (1997) 539 [hep-ph/9609331] [INSPIRE].

[196] R.-J. Zhang, Two loop effective potential calculation of the lightest CP even Higgs boson mass in the MSSM, Phys. Lett. B 447 (1999) 89 [hep-ph/9808299] [INSPIRE].

[197] S. Heinemeyer, W. Hollik and G. Weiglein, The Masses of the neutral CP-even Higgs bosons in the MSSM: accurate analysis at the two loop level, Eur. Phys. J. C 9 (1999) 343 [hep-ph/9812472] [INSPIRE].

[198] S. Heinemeyer, W. Hollik and G. Weiglein, The mass of the lightest MSSM Higgs boson: a compact analytical expression at the two loop level, Phys. Lett. B 455 (1999) 179 [hep-ph/9903404] [INSPIRE].

[199] J.R. Espinosa and R.-J. Zhang, MSSM lightest CP even Higgs boson mass to $O\left(\alpha_{s} \alpha_{t}\right)$ : the effective potential approach, JHEP 03 (2000) 026 [hep-ph/9912236] [INSPIRE].

[200] M.S. Carena et al., Reconciling the two loop diagrammatic and effective field theory computations of the mass of the lightest CP-even Higgs boson in the MSSM, Nucl. Phys. B 580 (2000) 29 [hep-ph/0001002] [INSPIRE].

[201] J.R. Espinosa and R.-J. Zhang, Complete two loop dominant corrections to the mass of the lightest CP-even Higgs boson in the minimal supersymmetric standard model, Nucl. Phys. B 586 (2000) 3 [hep-ph/0003246] [InSPIRE].

[202] S. Ambrosanio, A. Dedes, S. Heinemeyer, S. Su and G. Weiglein, Implications of the Higgs boson searches on different soft SUSY breaking scenarios, Nucl. Phys. B 624 (2002) 3 [hep-ph/0106255] [INSPIRE]. 
[203] A. Brignole, G. Degrassi, P. Slavich and F. Zwirner, On the $O\left(\alpha_{t}^{2}\right)$ two loop corrections to the neutral Higgs boson masses in the MSSM, Nucl. Phys. B 631 (2002) 195 [hep-ph/0112177] [INSPIRE].

[204] M.S. Carena and H.E. Haber, Higgs boson theory and phenomenology, Prog. Part. Nucl. Phys. 50 (2003) 63 [hep-ph/0208209] [INSPIRE].

[205] S.P. Martin, Complete two loop effective potential approximation to the lightest Higgs scalar boson mass in supersymmetry, Phys. Rev. D 67 (2003) 095012 [hep-ph/0211366] [INSPIRE].

[206] G. Degrassi, S. Heinemeyer, W. Hollik, P. Slavich and G. Weiglein, Towards high precision predictions for the MSSM Higgs sector, Eur. Phys. J. C 28 (2003) 133 [hep-ph/0212020] [INSPIRE].

[207] M. Frank et al., The Higgs boson masses and mixings of the complex MSSM in the Feynman-diagrammatic approach, JHEP 02 (2007) 047 [hep-ph/0611326] [INSPIRE].

[208] L.J. Hall, D. Pinner and J.T. Ruderman, A natural SUSY Higgs near 126 GeV, JHEP 04 (2012) 131 [arXiv:1112.2703] [InSPIRE].

[209] S. Heinemeyer, O. Stal and G. Weiglein, Interpreting the LHC Higgs search results in the MSSM, Phys. Lett. B 710 (2012) 201 [arXiv:1112.3026] [INSPIRE].

[210] P. Draper, P. Meade, M. Reece and D. Shih, Implications of a $125 \mathrm{GeV}$ Higgs for the MSSM and low-scale SUSY breaking, Phys. Rev. D 85 (2012) 095007 [arXiv:1112.3068] [inSPIRE].

[211] R. Barbieri, L.J. Hall, Y. Nomura and V.S. Rychkov, Supersymmetry without a light Higgs boson, Phys. Rev. D 75 (2007) 035007 [hep-ph/0607332] [INSPIRE].

[212] G. Degrassi and P. Slavich, On the radiative corrections to the neutral Higgs boson masses in the NMSSM, Nucl. Phys. B 825 (2010) 119 [arXiv:0907.4682] [INSPIRE].

[213] H.K. Dreiner, F. Staub and A. Vicente, General NMSSM signatures at the LHC, Phys. Rev. D 87 (2013) 035009 [arXiv:1211.6987] [INSPIRE].

[214] ATLAS collaboration, Search for associated production of the Higgs boson in the $W H \rightarrow W W W^{(*)} \rightarrow \ell \nu \ell \nu \ell \nu$ and $Z H \rightarrow Z W W^{(*)} \rightarrow \ell \ell \ell \nu \ell \nu$ channels with the ATLAS detector at the LHC, ATLAS-CONF-2013-075 (2013).

[215] ATLAS collaboration, Search for charged Higgs bosons in the $\tau+$ jets final state with $p p$ collision data recorded at $\sqrt{s}=8 \mathrm{TeV}$ with the ATLAS experiment, ATLAS-CONF-2013-090 (2013).

[216] A. Arbey, M. Battaglia, A. Djouadi and F. Mahmoudi, An update on the constraints on the phenomenological MSSM from the new LHC Higgs results, Phys. Lett. B 720 (2013) 153 [arXiv: 1211.4004] [INSPIRE].

[217] ATLAS collaboration, Measurements of Higgs boson production and couplings in the four-lepton channel in pp collisions at center-of-mass energies of 7 and $8 \mathrm{TeV}$ with the ATLAS detector, arXiv:1408.5191 [INSPIRE].

[218] SM Higgs branching ratios and partial-decay widths, CERN Report 3 (2013).

[219] LhC Higgs Cross Section Working Group collaboration, S. Heinemeyer et al., Handbook of LHC Higgs cross sections: 3. Higgs properties, arXiv:1307.1347 [INSPIRE].

[220] U. Ellwanger, A Higgs boson near $125 \mathrm{GeV}$ with enhanced di-photon signal in the NMSSM, JHEP 03 (2012) 044 [arXiv:1112.3548] [INSPIRE].

[221] K. Schmidt-Hoberg and F. Staub, Enhanced $h \rightarrow \gamma \gamma$ rate in MSSM singlet extensions, JHEP 10 (2012) 195 [arXiv:1208.1683] [INSPIRE]. 
[222] M. Carena, S. Gori, N.R. Shah, C.E.M. Wagner and L.-T. Wang, Light stau phenomenology and the Higgs $\gamma \gamma$ rate, JHEP 07 (2012) 175 [arXiv:1205.5842] [INSPIRE].

[223] J.A. Casas, J.M. Moreno, K. Rolbiecki and B. Zaldivar, Implications of light charginos for Higgs observables, LHC searches and dark matter, JHEP 09 (2013) 099 [arXiv:1305.3274] [INSPIRE].

[224] B. Batell, S. Jung and C.E.M. Wagner, Very light charginos and Higgs decays, JHEP 12 (2013) 075 [arXiv: 1309.2297] [INSPIRE].

[225] M. Hemeda, S. Khalil and S. Moretti, Light chargino effects onto $H \rightarrow \gamma \gamma$ in the MSSM, Phys. Rev. D 89 (2014) 011701 [arXiv:1312.2504] [INSPIRE]. 